$\underline{\text { Preprint typeset in JHEP style - PAPER VERSION }}$

hep-th/0507014

LPTHE-05-14

LPTENS-05-21

TIFR/TH/05-27

\title{
Precision Counting of Small Black Holes
}

\author{
Atish Dabholkar ${ }^{\mathrm{a}}$, Frederik Denef ${ }^{\mathrm{b}}$, Gregory W. Moore ${ }^{\mathrm{b}}$, and Boris Pioline ${ }^{\mathrm{c}, \mathrm{d}}$ \\ a Department of Theoretical Physics, Tata Institute of Fundamental Research, \\ Homi Bhabha Road, Mumbai 400005, India \\ ${ }^{\mathrm{b}}$ Department of Physics, Rutgers University, \\ Piscataway, NJ, 08854-8019 USA \\ ${ }^{\mathrm{c}}$ LPTHE, Universités Paris 6 et 7, 4 place Jussieu, \\ 75252 Paris cedex 05, France \\ ${ }^{\mathrm{d}}$ LPTENS, Département de Physique de l'ENS, 24 rue Lhomond, \\ 75231 Paris cedex 05, France \\ E-mail:atish@tifr.res.in, denef@physics.rutgers.edu, \\ gmoore@physics.rutgers.edu,pioline@lpthe.jussieu.fr
}

\begin{abstract}
It has recently been proposed that a class of supersymmetric higherderivative interactions in $\mathcal{N}=2$ supergravity may encapsulate an infinite number of finite size corrections to the microscopic entropy of certain supersymmetric black holes. If this proposal is correct, it allows one to probe the string theory description of black-hole micro-states to far greater accuracy than has been possible before. We test this proposal for "small" black holes whose microscopic degeneracies can be computed exactly by counting the corresponding perturbative BPS states. We also study the "black hole partition sum" using general properties of of BPS degeneracies. This complements and extends our earlier work in [1].
\end{abstract}




\section{Contents}

1. Introduction $\quad \mathbf{2}$

1.1 The Black Hole Attractor and the OSV Conjecture 3

1.2 Small Black Holes 5

1.3 Summary of Main Results 7

1.4 Outline of the Paper 11

2. A Benchmark $\mathcal{N}=4$ Example: Type $I I A / K 3 \times T^{2} \quad 12$

2.1 Review of Heterotic/Type II Duality in 4 Dimensions 12

2.2 Small Black Holes and DH States in the Het $(4,24)$ Model 14

2.3 Asymptotic Degeneracies and the Rademacher Formula $\quad 15$

2.4 Generalized Attractor Formalism for $\mathcal{N}=4$ and Leading Entropy $\quad 17$

2.5 Testing the OSV Formula 19

2.6 Degeneracies vs. Helicity Supertrace 21

2.7 DH states in type II $/ K 3 \times T^{2} \quad 25$

3. Small Black Holes in $\mathcal{N}=4$ Models with Reduced Rank 26

$3.1 \quad F_{1}$ in Reduced Rank Type II Models 26

3.2 Heterotic Duals and Exact Counting of DH States 28

3.3 A Detailed Analysis of the Het $(4,16)$ Model 29

3.4 General Reduced Rank Models 34

3.5 A Type II $(2,2) /(0,4)$ Dual Pair 35

4. Macroscopic Predictions for Extremal Black Holes Degeneracies in $\mathcal{N}=2$ Models $\quad 37$

4.1 Generalized Prepotential and the Topological String Amplitude 38

4.2 Large Black Holes with $p^{0}=0 \quad 40$

4.3 Small Black Holes 44

4.4 Large Black Holes with $p^{0} \neq 0 \quad 46$

5. Microscopic Counting of DH States in $\mathcal{N}=2$ Models 49

5.1 Het $/ K 3 \times T^{2}$ with Standard Embedding 49

5.2 Het $/ K 3 \times T^{2}$ with Symmetric Embedding 52

5.3 The $(2,12)$ FHSV Model 53

5.4 General $\mathcal{N}=2$ Asymmetric Orbifolds 60 
6. The Black Hole Partition Function 64

6.1 Rigid Divisors $\quad 65$

6.2 K3 Divisors in $K 3 \times T^{2} \quad 71$

6.3 General Case and Monodromy Invariance $\quad 72$

$\begin{array}{ll}\text { 7. Conclusion } & 75\end{array}$

$\begin{array}{ll}\text { A. The Rademacher Expansion } & 76\end{array}$

$\begin{array}{ll}\text { B. Modular Cornucopia } & 77\end{array}$

C. Counting $J=0$ DH States in the $(4,24)$ Model 79

D. Other $\operatorname{Het}\left(4, n_{V}\right)$ and $\operatorname{Het}\left(2, n_{V}\right)$ Models 82

E. Some Properties of the Mac-Mahon Function 91

\section{Introduction}

One of the distinct successes of string theory is that it explains the statistical origin of the thermodynamic Bekenstein-Hawking entropy [2-4] of certain supersymmetric black holes in terms of counting of underlying micro-states [5]. This has been particularly successful in the case of dyonic black holes in string theories with $\mathcal{N}=2$ supersymmetry in four dimensions. In the regime of large electric and magnetic charges, these black holes possess a non-singular horizon with area much larger than the Planck or string scale. For such "large" black holes, the Bekenstein-Hawking entropy, one quarter of the horizon area in Planck units, matches the logarithm of the number of micro-states of specific supersymmetric brane-configurations with the same quantum numbers $[6,7]$.

For black holes with large but finite area, there are subleading corrections to the Bekenstein-Hawking formula, due to higher-derivative interactions in the quantum effective action. The latter alter both the black hole geometry near the horizon, as well as the very relation between macroscopic entropy and geometry $[8,9]$. On the microscopic side, there are also finite size corrections to the entropy ${ }^{1}$, which however depend on the choice of a statistical ensemble. It is natural to ask whether the successful matching

\footnotetext{
${ }^{1}$ Following standard practice, we define the entropy as the Legendre transform of the logarithm of the partition function in a given statistical ensemble.
} 
between the Bekenstein-Hawking entropy and the string theoretical counting of black hole micro-states continues to hold beyond leading order.

Several advances in recent years have made it possible to address this question. By generalizing the attractor mechanism for $\mathcal{N}=2$ black holes, Cardoso, de Wit, and Mohaupt (CdWM) computed the Bekenstein-Hawking-Wald (BHW) entropy incorporating an infinite number of higher derivative F-type interactions [10-13]. Revisiting this result, Ooguri, Strominger and Vafa (OSV) conjectured that the statistical ensemble implicit in the CdWM entropy is a specific mixed ensemble [14], and furthermore that non F-type interactions can be consistently neglected provided one restricts to a suitable supersymmetric index on the microscopic side. If correct, this proposal opens the way to a more detailed comparison of macroscopic and microscopic degeneracies than has been possible thus far.

For a generic dyonic black hole, such a comparison is hampered by our insufficient understanding of the dynamics of the D-brane micro-states. The aim of this work is to identify and analyze a large class of examples where microscopic degeneracies are known exactly and where a very explicit comparison is possible exactly and to all orders in an asymptotic expansion. This complements and extends our earlier work [1] where some of the main results were announced. It should be noted that alternative approaches have been put forward [15-18], relying on different statistical ensembles. It goes beyond the scope of this paper to relate these two approaches.

\subsection{The Black Hole Attractor and the OSV Conjecture}

In general, the quantum effective admits an infinite series of unknown higher-derivative corrections, which make it difficult to determine higher-order corrections to the macroscopic entropy. In Type IIA string theory compactified on a Calabi-Yau three-fold $\mathcal{X}$ however, there exist an infinite series of computable higher-derivative F-term corrections of the form $F_{h}(X)\left({ }^{-} C^{-}\right)^{2}\left(T^{-}\right)^{2 h-2}$, where ${ }^{-} C^{-}$and $T^{-}$denote the anti-self-dual part of the Weyl tensor and graviphoton field strength, and $X^{I}$ the Kähler moduli of $\mathcal{X}$. The peculiarity of these interactions is that they can be written as the integral of a chiral density in superspace, and satisfy certain non-renormalisation properties. In particular, they arise only at genus $h$ in type II string theory, and the coefficient $F_{h}\left(X^{I}\right)$ reduces to the genus $h$ vacuum amplitude in the A-model topological string on $\mathcal{X}[19,20]$. The Bekenstein-Hawking-Wald macroscopic entropy of BPS black holes incorporating these interactions was computed by CdWM in [10-13], generalizing the standard tree-level attractor mechanism. As noticed by the authors of [14], this expression takes a particularly simple form after Legendre transform with respect to the 
electric charges ${ }^{2}$

$$
S_{\mathrm{CdWM}}\left(p^{I}, q_{I}\right)=\mathcal{F}_{\text {top }}\left(p^{I}, \phi^{I}\right)+\pi \phi^{I} q_{I}, \quad \pi q_{I}=-\frac{\partial}{\partial \phi^{I}} \mathcal{F}_{\text {top }}\left(p^{I}, \phi^{I}\right)
$$

where

$$
\mathcal{F}_{\text {top }}\left(p^{I}, \phi^{I}\right):=-\pi \operatorname{Im}\left[F_{\text {top }}\left(p^{I}+i \phi^{I}, 2^{8}\right)\right] .
$$

is proportional to the imaginary part of the all-order topological string vacuum amplitude $F_{\text {top }}\left(X, W^{2}\right)=\sum_{h=0}^{\infty} W^{2 h-2} F_{h}\left(X^{I}\right)$ evaluated at $X^{I}=p^{I}+i \phi^{I}$ and $W^{2}=2^{8}$. The attractor equations [22-24]

$$
p^{I}=\operatorname{Re}\left(X^{I}\right), \quad q_{I}=\operatorname{Re}\left(\partial F_{t o p} / \partial X^{I}\right)
$$

controlling the fixed point behavior of the Kähler moduli at the horizon follow naturally from this procedure [14].

Based on this observation, Ooguri, Strominger and Vafa (OSV) have proposed that the statistical ensemble implicit in the above Bekenstein-Hawking-Wald entropy has fixed magnetic charges $p^{I}$, but fluctuating electric charges $q_{I}$ at a fixed electric potential $\phi^{I}[14]$ :

$$
Z_{O S V}\left(p^{I}, \phi^{I}\right):=\exp \mathcal{F}_{O S V}\left(p^{I}, \phi^{I}\right):=\sum_{q^{I} \in \Lambda_{e}} \Omega\left(p^{I}, q_{I}\right) e^{\pi \phi^{I} q_{I}}
$$

where $\Omega\left(p^{I}, q_{I}\right)$ denotes the number or possibly a suitable index of micro-states with electric and magnetic charges $q_{I}$ and $p^{I}$, and $\Lambda_{e}$ is the lattice of electric charges in the large volume polarization. Put otherwise, the essence of the proposal [14] is an equality between the microscopic free energy $\mathcal{F}_{O S V}$ in the mixed statistical ensemble (1.4) and the macroscopic free energy $\mathcal{F}_{\text {top }}$ computed from the higher-derivative F-term interactions,

$$
\mathcal{F}_{\text {OSV }}\left(p^{I}, \phi^{I}\right) \equiv \mathcal{F}_{\text {top }}\left(p^{I}, \phi^{I}\right)
$$

Using the relation (1.2) between the topological free energy $\mathcal{F}_{\text {top }}$ and the topological string amplitude $F_{t o p}$, this equation may be rephrased as a relation between the BPS black hole degeneracies in type II on $\mathcal{X}$ and the topological string amplitude,

$$
Z_{O S V}\left(p^{I}, \phi^{I}\right) \equiv\left|\exp \left[\frac{i \pi}{2} F_{t o p}\left(p^{I}+i \phi^{I}, 2^{8}\right)\right]\right|^{2} .
$$

Evaluating the sum over charges in the partition function (1.4) by steepest descent, one indeed finds that the Legendre transform of the entropy is equal to the topological free energy (1.2), in the limit of large charges.

\footnotetext{
${ }^{2}$ The generality of this fact has been recently clarified in [21].
} 
The proposal (1.5) goes far beyond the large charge regime in which it was motivated, since it allows in principle to extract the microscopic degeneracies of BPS black holes from the topological string amplitude by means of an inverse Laplace transform,

$$
\Omega\left(p^{I}, q_{I}\right) \equiv \int\left[d \phi^{I}\right] \exp \left[\mathcal{F}_{\text {top }}\left(p^{I}, \phi^{I}\right)+\pi q_{I} \phi^{I}\right]
$$

A strong form of the conjecture asserts that this equation holds at finite electric and magnetic charges, provided some yet unknown non-perturbative contributions to the topological string amplitude are included [14]. A weaker form states that this equality hold to all orders in an asymptotic expansion in the inverse of the charges [14]. One aim of our work is make Eqs. (1.7),(1.6) more precise and use them to study the degeneracies of finite charge black holes. Certain proposed nonperturbative corrections to (1.7) have been explored in $[25,26]$, but in a rather different context from the examples studied here.

\subsection{Small Black Holes}

For this purpose, it is useful to consider cases for which the exact degeneracies of the micro-states are computable. Using heterotic / type II duality, this is indeed possible for type II black holes which are dual to the heterotic Dabholkar-Harvey (DH) states $[27,28]$. Recall that these are BPS states in the perturbative heterotic spectrum, which exist provided the conformal field theory contains a compact free boson. The simplest example is provided by a state carrying quantized momentum $n$ and winding number $w$ around an internal circle. The left- and right-moving momenta are given by

$$
q_{R, L} \equiv \sqrt{\frac{\alpha^{\prime}}{2}}\left(\frac{n}{R} \pm \frac{w R}{\alpha^{\prime}}\right)
$$

and the vector $\left(q_{R}, q_{L}\right)$ belongs to the Narain lattice $\Gamma^{1,1}$. Such a state is half-BPS as long as it is in the right-moving superconformal ground state but it can carry arbitrary left-moving excitations that satisfy the level-matching

$$
N-1=\frac{1}{2}\left(q_{R}^{2}-q_{L}^{2}\right)=n w,
$$

where $N$ is the left-moving excitation level. For given charges $(n, w)$, there is a Hagedorn density $\Omega(n, w) \sim \exp (4 \pi \sqrt{|n w|})$ of such states, as a result of the large degeneracy of the left-moving excitations.

The integers $(n, w)$ can be viewed as the quantized electric charges under the Kaluza-Klein and Neveu-Schwarz gauge fields $g_{\mu i}$ and $B_{\mu i}$ arising by dimensional reduction along the circle. The mass $M$ of the state $(n, w)$ saturates the BPS bound

$$
M^{2}=q_{R}^{2}=\left[\frac{n}{R}+\frac{w R}{\alpha^{\prime}}\right]^{2}=\frac{n^{2}}{R^{2}}+\frac{w^{2} R^{2}}{\alpha^{\prime 2}}+\frac{2(N-1)}{\alpha^{\prime}}
$$


where $R$ is the radius of the circle. Provided it does not become degenerate with another half-BPS state with which it may pair up, the $(n, w)$ state is therefore stable. As the string coupling $g_{H}$ is increased, the de Broglie - Compton wavelength $1 / M$ of the particle becomes smaller than its Schwarzschild radius $M l_{P}^{2}$, leading to the formation of an extremal black hole with electric charges $(n, w)$. It is thus tempting to compare the Bekenstein-Hawking entropy of this black hole with the logarithm of the number of fundamental strings with the same charges [29-33],

$$
S_{D H}=\log \Omega(n, w) \sim 4 \pi \sqrt{|n w|}
$$

More generally, the black hole charges are characterized by an arbitrary charge vector $Q$ in the Narain lattice $\Gamma^{6,22}$ and the leading entropy of the DH states in that case goes as $S_{D H} \sim 4 \pi \sqrt{Q^{2} / 2}$.

In contrast with the "large" black holes discussed above, these black holes are singular solutions of the tree-level supergravity Lagrangian [34,35], where the horizon and the inner singularity coalesce. Their classical entropy therefore vanishes, as a result of their carrying only electric charge (in the natural heterotic polarization). While the heterotic string coupling goes to zero at the singularity, higher-derivative $\alpha^{\prime}$ corrections are however expected to be quite important, and, assuming the singularity is resolved, have been argued to lead to an entropy of the required order [32]. By including the tree-level $R^{2}$ correction to the heterotic effective Lagrangian (or, from the type II point of view, the large volume limit of the one-loop topological amplitude $F_{1}$ ), it was shown recently that the black hole develops a smooth horizon, with a similar geometry $A d S_{2} \times S^{2} \times \mathcal{X}$ as found in the large black hole case [36,37] (see [13,38,39] for earlier work on this subject). Moreover, the Bekenstein-Hawking-Wald entropy, taking into account this $R^{2}$ correction, matches the microscopic entropy in leading order, including the precise numerical coefficient [36,37]. The geometry interpolating between the horizon and infinity has been recently studied in $[15,40]$. For this type of black hole, the four-dimensional heterotic string coupling is of order $g_{H}^{2} \sim 1 / \sqrt{|n w|}$ at the horizon, so that the area is of the same order as the inverse tension of the heterotic string $l_{H}^{2}=l_{P}^{2} / g_{H}^{2}$ at the horizon. We shall thus refer to these states as "small" black holes, keeping in mind that, for large charges, they are nevertheless much larger than the Planck scale.

Recently, the OSV conjecture has been tested for small black holes in type IIA string theory compactified on $K 3 \times T^{2}$, or equivalently, in heterotic string theory on $T^{6}$ [37]. Although the original proposal was formulated for $\mathcal{N}=2$ backgrounds, an extension to the $\mathcal{N}=4$ case is simpler to analyze since all gravitational F-terms vanish except $F_{1}$ [41]. Using the generalized attractor formalism in [10-13], adapted to the $\mathcal{N}=4$ setting, it was found that the macroscopic entropy of these small black holes 
precisely matches the Ramanujan-Hardy estimate for the number of heterotic BPS states preserving 1/2 supersymmetry [37]. It was also shown that even the sub-leading corrections to the entropy computed using the OSV proposal match to all orders in an asymptotic expansion. The super-gravity solutions for these small black holes have been further analyzed in $[15,36,40,42]$.

In this paper, we greatly extend the range of validity of the analysis in [37], by studying the exact degeneracy of small black holes in a variety of backgrounds with $\mathcal{N}=4$ supersymmetry (but a different low-energy spectrum from the "benchmark" $K 3 \times T^{2}$ case), or with $\mathcal{N}=2$ supersymmetry (for Calabi-Yau compactifications with a $K 3$ fibration).

\subsection{Summary of Main Results}

For the reader's convenience, we summarize our main results below:

1. On the heterotic side, by standard orbifold techniques, the microscopic degeneracy of the DH states can be enumerated using modular forms. The leading microscopic entropy at large charge can be extracted using the Hardy-Ramanujan formula. The Rademacher formula provides a convenient way to extract subleading corrections: it expresses the Fourier coefficients of the modular form as a series of modified Bessel functions, where each term is exponentially suppressed (but nevertheless exponentially growing) with respect to the previous one (see [43] for a review). In particular, all power corrections to the leading entropy are captured by the first Bessel function in the Rademacher expansion.

2. Retaining only the perturbative part of the topological amplitude (i.e. discarding the Gromov-Witten instanton series), and assuming a proper choice of contour, we find that the integral (1.7) can be computed exactly, both in the large and small black hole case, and expressed as a modified Bessel function of the first kind. Using the standard asymptotic expansion of the latter, the leading term

is the Bekenstein-Hawking-Wald entropy $S_{B H W}=4 \pi \sqrt{Q^{2} / 2}$ predicted by the generalized attractor mechanism. In particular, due to the topological coupling $F_{1}$, the entropy of small black holes is computable and finite, as observed in [37]. In addition, the Bessel function captures an infinite number of computable corrections in inverse powers of the charges.

3. In a variety of $\mathcal{N}=4$ and $\mathcal{N}=2$ models, we find that the integral (1.7), neglecting the Gromov-Witten instanton series, reproduces precisely the leading Bessel function in the Rademacher expansion of the degeneracies of heterotic DH states. In other words, the OSV proposal predicts the correct degeneracies of BPS states, 
to all orders in an asymptotic expansion in inverse powers of the charges. In particular, the leading entropy is correctly reproduced, including the corrections computed in [44]. Importantly, this success relies only on the large volume limit of $F_{1}$ only (equivalently, on the heterotic tree-level $R^{2}$ amplitude).

4. For this all order perturbative agreement to hold, it is important to use the holomorphic topological amplitude, which controls the Wilsonian supergravity action, rather than the non-holomorphic BCOV generating function, which describes the 1PI couplings in the low-energy effective action. This is consistent with the discussion in [45], but in stark contrast with the alternative approaches in $[16,18]$ (note however that [45] has proposed a formally equivalent formula, using the holomorphic rather than real polarization, where non-holomorphic anomalies are likely to play a role). It is also important to count states with arbitrary angular momentum $J$, as the restriction to $J=0$ states leads to different subleading terms in the microscopic amplitude, which would spoil agreement with the OSV prediction. In other words, the proper statistical ensemble implicit in the Bekenstein-Hawking-Wald entropy appears to be an ensemble with zero angular velocity at the horizon, rather than zero angular momentum. Finally, it is necessary to consider ratios of degeneracies at fixed magnetic charge only, in order to cancel a magnetic-charge dependent pre-factor $\mathcal{N}(p)$, which would spoil duality invariance. For $p^{0} \neq 0$, a more drastic modification is necessary, since, as shown in Section 4.4, the pre-factor in general involves both electric and magnetic charges.

5. The neglect of Gromov-Witten instantons can be rigorously justified in $\mathcal{N}=4$ cases, as all instanton corrections are exponentially suppressed. The situation is more subtle in $\mathcal{N}=2$ theories: when $\chi(\mathcal{X}) \neq 0$, the series of point-like instantons contribution becomes strongly coupled in the regime of validity of the Rademacher formula, $\hat{q}_{0} \gg \hat{C}(p)$. The strong coupling behavior is controlled, up to a logarithmic term, by the Mac-Mahon function, which is exponentially suppressed in this regime. Upon absorbing the logarithmic term into a redefinition of the topological string amplitude $\Psi_{\text {top }} \rightarrow \lambda^{\chi / 24} \Psi_{\text {top }}$, one recovers the naive result. As for non-degenerate instantons, they are exponentially suppressed provided all magnetic charges are non zero. This is unfortunately not the case for the small black holes dual to the heterotic DH states, whose Kähler classes are attracted to the boundary of the Kähler cone at the horizon. In this case, we cannot rigorously justify the neglect of Gromov-Witten contributions.

6. Even in the cases where an all-order agreement is obtained, the OSV formula appears to fail in reproducing the subleading Bessel functions in the Rademacher 
expansion of the microscopic degeneracies, as those cannot be associated to subleading saddle points in the contour integral (1.7) in any obvious way ${ }^{3}$. As a matter of fact, we encounter serious difficulties in trying to make sense of the formula (1.7) non-perturbatively. Due to the non-convexity of the free energy $\mathcal{F}$ (or, equivalently, the instability of the mixed thermodynamical ensemble), the convergence of the integral can only be achieved when the potentials $\phi^{I}$ take imaginary values. However, at least for Calabi-Yau threefolds admitting a $K 3$ fibration, the topological string amplitude $\Psi_{\text {top }}$ is an automorphic form, and is very badly behaved at the boundary of moduli space where the moduli $X^{I}$ become real.

7. On general grounds (e.g. if it is to satisfy the second law of thermodynamics), the Bekenstein-Hawking-Wald entropy, including all higher-derivative corrections, is expected to be equal to the logarithm of the total number of micro-states. The truncation to only F-term type higher-derivative corrections is not expected to have a thermodynamical interpretation, unless non-F-terms do not contribute by some non-renormalization property ${ }^{4}$. On the other hand, the counting of heterotic DH states at zero string coupling may differ from the actual number of states in the regime where a black hole is formed, due to the possibility of BPS states pairing up into longer multiplets. Useful diagnostic tools to determine whether this happens are helicity supertraces $\Omega_{n}=\operatorname{Tr}(-1)^{F} J_{3}^{n}$ (where $F$ is the space-time fermion number and $J_{3}$ one of the generators of the little-group of a massive particle in 4 dimensions), namely $\Omega_{2}$ for $1 / 2$ BPS states in theories with $\mathcal{N}=2$ supersymmetry, $\Omega_{4}$ for $1 / 2$ BPS states in theories with $\mathcal{N}=4$ and $\Omega_{6}$ for $1 / 4$ BPS states in theories with $\mathcal{N}=4$. In contrast to absolute degeneracies, helicity supertraces are invariant under generic variations of the moduli (except for lines of marginal stability). If cases where the degeneracies at zero coupling can be identified with an helicity supertrace, one can reasonably assume that they will be equal to the actual number of states in the black hole regime (barring the unlikely possibility that long multiplets unpair as the coupling is increased). We can then reliably compare them with the macroscopic BHW entropy ${ }^{5}$. In some cases however, the helicity supertraces can be exponentially smaller than the zero-

\footnotetext{
${ }^{3}$ It was recently proposed that exponentially suppressed contributions should reflect multi-centered black hole configurations [46].

${ }^{4}$ See [47] for some recent interesting results in this direction.

${ }^{5}$ This differs from the interpretation advocated in [14], who propose to identify directly the topological amplitude with a supersymmetric index. This is a mathematically appealing and logically acceptable conjecture, but it has no direct bearing on the relation between the BHW entropy and the counting of black hole micro-states.
} 
coupling degeneracies ${ }^{6}$, and it is difficult to determine the actual number of states at strong coupling.

8. We find that in cases where the absolute degeneracies are equal to the helicity supertraces, the instanton-deprived OSV proposal appears to work successfully. This includes $1 / 2$ BPS states in all $\mathcal{N}=4$ models, as well as BPS states in twisted sectors of $\mathcal{N}=2$ orbifolds. This suggests that, for this class of BPS black holes, non F-type higher derivative interactions have no effect, if not on the geometry, at least on the Bekenstein-Hawking-Wald entropy. It would be very interesting to check such a non-renormalization explicitly.

9. In cases where it appears to fail, the helicity supertraces are in general exponentially smaller than their absolute degeneracy, due to cancellations of pairs of $\mathrm{DH}$ states. This occurs in general for (i) untwisted DH states of $\mathcal{N}=2$ heterotic orbifolds, and (ii) DH states in $\mathcal{N}=4$ type II orbifolds. In case (i), the OSV prediction appears to agree with the absolute degeneracies of untwisted DH states to leading order (which have the same exponential growth as twisted DH states), but not at subleading order (as the subleading corrections in the untwisted sector are moduli-dependent, and uniformly smaller than in the twisted sectors). In models where twisted and untwisted states cannot be distinguished by their charges, the helicity supertrace $\Omega_{2}$ is dominated by the contribution of the twisted sectors, and it may be consistent to identify it with the l.h.s. of (1.7). The situation in case (ii) is rather different, since the helicity supertraces grow only as a power rather than exponentially. On the macroscopic side, $R^{2}$ interactions are not sufficient to resolve the singular horizon, and higher derivative interactions are bound to become important.

10. Conversely, one may try to compute the black hole partition function (1.4) from our knowledge of the microscopic degeneracies, and compare to the proposed answer (1.6). For some choices of Calabi-Yau manifolds and of magnetic charges, in the infinite radius limit, the degeneracies are known exactly for arbitrary electric charges, and this program can be carried out explicitly. Examples of this are D4-D2-D0 bound states wrapped on a rigid divisor, or D-branes dual to heterotic DH states.

An immediate problem which arises when attempting to compute the partition sum (1.4) is that it is badly divergent. We solve this by introducing a convenient and physically natural regulator, namely an additional Boltzmann weight

${ }^{6}$ This occurs e.g. in the case of $5 \mathrm{D}$ black holes [48], but we shall find numerous other examples in this work. 
$e^{-\alpha H(p, q)}$, with $H(p, q)$ the BPS energy of the given charge. This renders the partition function finite and rigorously justifies various formal manipulations, after which one can send $\alpha$ back to zero.

Our result is that in these cases, the polynomial part of the resulting free energy indeed equals the corresponding terms at the right-hand side of (1.6), but with an additional sum over integral imaginary shifts of the $\phi^{a}$ on the right hand side. This ensures periodicity under $\phi^{a} \rightarrow \phi^{a}+2 i n^{a}$, as is required by the definition (1.4). In fact this summed version of (1.6) is trivially equivalent to the integral form (1.7), with the $\phi^{a}$ integration contours running over the entire imaginary axis.

More importantly, we find that at least for these choices of charges, the nonperturbative part of the topological string free energy is not reproduced; the corrections to the polynomial terms of both sides do not match. This is true even in the limit of large charges.

11. Despite the fact that the exact degeneracies are not known in more general cases, one can extract some information about the general partition function by exploiting large radius monodromy invariance. These are integral shifts of the NS B-field, acting on the $\phi^{a}$ as $\phi^{a} \rightarrow \phi^{a}+n^{a} \phi^{0}$. This induces a spectral flow on the electric and magnetic charges, which leaves the degeneracies unchanged, at least in the large volume limit. Exploiting this symmetry, we argue that the BPS partition sum does not generate the full data of the topological amplitude at any finite magnetic charge $P$. In particular we show that the $\phi^{a}$-dependence of the integrand in (1.7) predicted from monodromy invariant BPS degeneracies is simply given by a finite sum of Gaussians, which is to be compared to the intricate $\phi^{a}$-dependence generated by the Gromov-Witten series in the topological string free energy. The conjecture might still hold in a suitable asymptotic sense when $P \rightarrow \infty$, because in this case number of independent Gaussian terms will in general go to infinity.

\subsection{Outline of the Paper}

This paper is organized as follows.

In Section 2, we illustrate our methods in the simplest example with $\mathcal{N}=4$ supersymmetry: type IIA string theory compactified on $K 3 \times T^{2}$, or equivalently, in heterotic string theory compactified on $T^{6}$, extending the analysis in [37].

In Section 3, we generalize this analysis to a class of $\mathcal{N}=4$ models with reduced rank, obtained as freely acting orbifolds of the IIA $/ K 3 \times T^{2}$ or Het $/ T^{6}$ models. 
In Section 4, we come to the $\mathcal{N}=2$ supersymmetric case, for which the OSV conjecture was originally formulated. After recalling the main features of the topological string amplitude, we compute the asymptotic degeneracies predicted by (1.7) for a particular scaling of the charges.

In Section 5, we compare this prediction to the microscopic counting in the perturbative heterotic description. After discussing several illustrative $\mathcal{N}=2$ models, we find the asymptotic degeneracies of DH states for arbitrary asymmetric orbifold of the heterotic string compactified on $T^{6}$.

In Section 6, we reverse the approach, construct the partition function in the mixed thermodynamical ensemble (1.4) from our partial knowledge of the micro-canonical degeneracies, and compare the result to the topological string amplitude.

Section 7 contains our conclusions and further comments.

In the Appendices, the reader will find a summary of the Rademacher expansion for the Fourier coefficients of modular forms with negative weight (Appendix A), a collection of useful modular identities (B), an analysis of the degeneracies of the DH states at fixed angular momentum in the Het $/ T^{6}$ model $(\mathrm{C})$ and a detailed computation of the degeneracies of $\mathrm{DH}$ states in $\mathcal{N}=4$ and $\mathcal{N}=2$ orbifolds of the $S O(32)$ heterotic string (D), a detailed analysis of the asymptotic expansion of the Mac-Mahon as well as an observation on its (non-)modularity (E).

\section{A Benchmark $\mathcal{N}=4$ Example: Type $I I A / K 3 \times T^{2}$}

In this section, we revisit the "benchmark" case of small black holes in type IIA string theory compactified on $K 3 \times T^{2}$, or equivalently heterotic string compactified on $T^{6}$, first discussed in [37]. Despite the fact that this model has $\mathcal{N}=4$ supersymmetry, we shall be able to apply the $\mathcal{N}=2$ attractor formalism, provided 4 out of the 28 charges, corresponding to gauge fields in gravitino multiplets of $\mathcal{N}=2$ supersymmetry,

vanish. For this reason we shall denote this model as the Het/IIA $(4,24)$, where the first number refers to the number of supersymmetries in 4 dimensions, and the second to the effective number of $\mathcal{N}=2$ vector multiplets, including the graviphoton. More general Het/IIA $\left(4, n_{V}\right)$ compactifications with $\mathcal{N}=4$ supersymmetry and $n_{V}<24$ vector multiplets will be discussed in Section 3 and Appendix D.

\subsection{Review of Heterotic/Type II Duality in 4 Dimensions}

Let us consider the type IIA string compactified on $K 3 \times T^{2}$. The massless spectrum consists of the $\mathcal{N}=4$ supergravity multiplet together with 22 vector multiplets. The 
moduli space takes a factorized form

$$
\frac{S L(2, \mathbb{R})}{U(1)} \times \frac{S O\left(6, n_{V}-2, \mathbb{R}\right)}{S O(6) \times S O\left(n_{V}-2\right)}
$$

with $n_{V}=24$, where the first factor corresponds to the Kähler modulus $T$ of $T^{2}$, while the axio-dilaton $S$, the complex structure modulus $U$ of $T^{2}$ and the geometric moduli of $K 3$ sit in the second factor. Points in (2.1) related by an action of the duality group $S l(2, \mathbb{Z}) \times O\left(\Gamma_{6,22}\right)$ are non-perturbatively equivalent. The gauge fields in the 22 vector multiplets originate from the 3 -form gauge field in the ten-dimensional type IIA string, after reduction on a basis $\gamma_{a}, a=2 \ldots 24$ of 2-cycles in $H^{2}(K 3, \mathbb{R})$. Accordingly, the electrically charged states are D2-branes wrapped on 2-cycles $\gamma_{a}$, and their magnetic counterparts are D4-branes wrapped on $T^{2} \times \gamma_{a}$, with charges $\left(q_{a}, p^{a}\right)$, respectively. On the other hand, the 6 gauge fields in the $\mathcal{N}=4$ supergravity multiplet correspond to the ten-dimensional Ramond-Ramond (RR) 1-form, the 3-form reduced on $T^{2}$, the KalbRamond 2-form reduced on either circle of $T^{2}$ and the Kaluza-Klein gauge fields on $T^{2}$. The corresponding electric charges are therefore carried by the D0-brane (denoted by $\left.q_{0}\right)$, D2-brane wrapped on $T^{2}\left(q_{1}\right)$, the fundamental string wrapped on $S^{1} \subset T^{2}$ $\left(w^{5}, w^{6}\right)$ and the momentum states on $T^{2}\left(n_{5}, n_{6}\right)$, respectively; the magnetic charges are carried by the D6-brane wrapped on $K 3 \times T^{2}\left(p^{0}\right)$, the D4-brane wrapped on $K 3$ $\left(p^{1}\right)$, the NS5-branes wrapped on $K 3 \times S^{1}\left(m^{5}, m^{6}\right)$ and the Kaluza-Klein monopoles on $S^{1} \subset T^{2}\left(k^{5}, k^{6}\right)$.

One of the earliest string duality conjectures identifies this model with the heterotic string compactified on $T^{6}$. The massless spectrum is identical, but the $S l(2) / U(1)$ complex scalar in the supergravity multiplet is now the heterotic axio-dilaton. The second factor in (2.1) is identified as the Narain moduli space of the even self-dual compactification lattice $\Gamma_{6,22}$. The 28 charges now correspond to the Cartan subalgebra of the rank 16 ten-dimensional gauge group, the reduction of the Kalb-Ramond twoform on $T^{6}$ and the Kaluza-Klein gauge fields on $T^{6}$. Accordingly, the electric charges in the natural heterotic polarization are carried by the 10-dimensional charged states, the fundamental string wound around $S^{1} \subset T^{6}$ and the momentum states along $S^{1} \subset T^{6}$; the corresponding magnetic charges are carried by H-monopoles, NS5-branes and KK5monopoles wrapped on $T^{5} \subset T^{6}$. The precise map can be obtained by applying triality on an $S O(4,4)$ subgroup of the $S O(4,20)$ duality group in 6 dimensions [49], and is displayed in Table 2.1 below. In particular, the $S O(6,22)$ vectors $Q, P$ of electric and magnetic charges in the natural heterotic polarization are related to the type II charges by

$$
\begin{aligned}
& Q=\left(q_{0}, p^{1}, q_{a}, n_{5}, n_{6}, m^{5}, m^{6}\right) \\
& P=\left(-q_{1}, p^{0}, C_{a b} p^{b},-w^{6}, w^{5}, k^{5}, k^{6}\right)
\end{aligned}
$$




\begin{tabular}{|c|c||l|l||l|l|}
\hline \multicolumn{2}{|c||}{$H e t / T^{6}$} & \multicolumn{2}{c||}{$I I A / K 3 \times T^{2}$} & \multicolumn{2}{c|}{ Charges } \\
\hline$K K / 1$ & $N S 5 / \hat{1}$ & $D 0$ & $D 2 / T^{2}$ & $q_{0}$ & $q_{1}$ \\
$K K / 2,3,4$ & $N S 5 / \hat{2}, \hat{3}, \hat{4}$ & $D 2 / \gamma_{a}$ & $D 4 / T^{2} \times \gamma_{a}$ & $q_{a=2,3,4}$ & $p^{a=2,3,4}$ \\
$K K / 5,6$ & $N S 5 / 6,5$ & $K K / 5,6$ & $F 1 / 6,5$ & $n_{5}, n_{6}$ & $-w^{6}, w^{5}$ \\
\hline$F 1 / 1$ & $K K M / \hat{1}$ & $D 4 / K 3$ & $D 6 / K 3 \times T^{2}$ & $p^{1}$ & $p^{0}$ \\
$F 1 / 2,3,4$ & $K K M / \hat{2}, \hat{3}, \hat{4}$ & $D 2 / \gamma_{a}$ & $D 4 / T^{2} \times \gamma_{a}$ & $q_{a=5,6,7}$ & $p^{a=5,6,7}$ \\
$F 1 / 5,6$ & $K K M / \hat{5}, \hat{6}$ & $N S 5 / \hat{6}, \hat{5}$ & $K K M / \hat{5}, \hat{6}$ & $m^{5}, m^{6}$ & $k^{5}, k^{6}$ \\
\hline$Q_{1, \ldots, 16}$ & $H M_{1, \ldots, 16}$ & $D 2 / \gamma_{a}$ & $D 4 / T^{2} \times \gamma_{a}$ & $q_{a=8, \ldots, 23}$ & $p^{a=8, \ldots, 23}$ \\
\hline
\end{tabular}

Table 1: Charge assignment in the Het/IIA $(4,24)$ model. The vertical columns denote $O(6,22)$ vectors. Even and odd columns are related by the Weyl reflection in $S l(2, \mathbb{Z})$, i.e. S-duality on the heterotic side or double T-duality on $T^{2}$ followed by an exchange of the two circles on the type II side. Abbreviations: KK/1= momentum state along $S^{1}, N S 5 / \hat{1}=$ NS5-brane wrapped on all directions except $1, \mathrm{KKM} / \hat{5}=$ Kaluza-Klein monopole localized in direction 5, $\mathrm{HM}=\mathrm{H}$-monopole.

with $S O(6,22)$ invariant inner products

$$
\begin{aligned}
Q^{2} & =2 q_{0} p^{1}+q_{a} C^{a b} q_{b}+2 m^{i} n_{i} \\
P^{2} & =-2 q_{1} p^{0}+p^{a} C_{a b} p^{b}+2 \epsilon_{i j} w^{i} k^{j} \\
Q \cdot P & =p^{0} q_{0}-p^{1} q_{1}+p^{a} q_{a}+n_{i} k^{j}+\epsilon_{i j} m^{i} w^{j}
\end{aligned}
$$

The heterotic polarization is therefore obtained from the type II large volume polarization by applying electric-magnetic duality to the $(D 4 / K 3, D 2 / T 2)$ and $(F 1, N S 5 / K 3 \times$ $\left.S^{1}\right)$ pairs.

\subsection{Small Black Holes and DH States in the Het $(4,24)$ Model}

The tree-level Bekenstein-Hawking entropy for generic BPS black holes in models with $\mathcal{N}=4$ supersymmetry is given by

$$
S_{B H}=\pi \sqrt{(P \cdot P)(Q \cdot Q)-(P \cdot Q)^{2}}
$$

in the natural heterotic polarization, such that $P, Q$ transform as a doublet of $S O\left(6, n_{V}-\right.$ 2 ) vectors under $S l(2)$ [50]. We shall be interested in black holes which are dual to perturbative heterotic states, with vanishing magnetic charge $P=0$, hence zero tree-level entropy. In particular, let us consider a type IIA state with $q^{0}$ D0-brane charge and $p_{1}$ D4-brane charge. This is dual to a fundamental heterotic string with momentum $n=q_{0}$ and winding $w=p^{1}$ along one circle in $T^{6}$. As we reviewed in Section 2.1, DH heterotic states with these charges can be obtained by tensoring the ground state of 
the right-moving superconformal theory with a level $N$ excitation of the 24 left-moving bosons, provided the level matching condition $N-1=n w$ is satisfied. The number of distinct DH states with fixed charges $(n, w)$ is thus $\Omega(n, w)=p_{24}(N)$, where $p_{24}(N)$ is the number of partitions on $N$ into the sum of 24 integers (up to an overall factor of 16 corresponding to the size of short $\mathcal{N}=4$ multiplets, which we will always drop). Accordingly, the generating function of the degeneracies of DH states is

$$
\sum_{N=0}^{\infty} p_{24}(N) q^{N-1}=\frac{1}{\Delta(q)}
$$

where $\Delta(q)$ is Jacobi's discriminant function

$$
\Delta(q)=\eta^{24}(q)=q \prod_{n=1}^{\infty}\left(1-q^{n}\right)
$$

It should be noted that the partition function for the degeneracies of the $D 0-D 4$ system can be obtained without resorting to the dual heterotic formulation, either by computing the Euler number of the Hilbert scheme of $K 3$, or by enumerating genus $g$ curves in $K 3$ [51,52]. Nevertheless, the heterotic description will prove very useful in more complicated examples. Notice that the type IIA model on $K 3 \times T^{2}$ also has DH states with zero tree-level entropy, but those are in general 1/4-BPS. We shall return to them in 2.7 .

\subsection{Asymptotic Degeneracies and the Rademacher Formula}

In order to determine the asymptotic density of states at large $N-1=n w$, it is convenient to extract $d(N)$ from the partition function (2.6) by an inverse Laplace transform,

$$
p_{24}(N)=\frac{1}{2 \pi i} \int_{\epsilon-i \pi}^{\epsilon+i \pi} d \beta e^{\beta(N-1)} \frac{16}{\Delta\left(e^{-\beta}\right)} .
$$

where the contour $C$ runs from $\epsilon-i \pi$ to $\epsilon+i \pi$, parallel to the imaginary axis. One may now take the high temperature limit $\epsilon \rightarrow 0$, and use the modular property of the discriminant function (see Appendix B)

$$
\Delta\left(e^{-\beta}\right)=\left(\frac{\beta}{2 \pi}\right)^{-12} \Delta\left(e^{-4 \pi^{2} / \beta}\right) .
$$

As $e^{-4 \pi^{2} / \beta} \rightarrow 0$, we can approximate $\Delta(q) \sim q$ and write the integral as

$$
p_{24}(N)=\frac{16}{2 \pi i} \int_{C} d \beta\left(\frac{\beta}{2 \pi}\right)^{12} e^{\beta(N-1)+4 \frac{\pi^{2}}{\beta}}
$$


This integral may be evaluated by steepest descent: the saddle point occurs at $\beta=$ $2 \pi / \sqrt{N-1}$, leading to the characteristic exponential growth $p_{24}(N) \sim \exp (4 \pi \sqrt{n w})$ for the degeneracies.

To calculate the sub-leading terms systematically in an asymptotic expansion at large $N$, one may recognize that (2.10) is proportional to the integral representation of a modified Bessel function,

$$
I_{\nu}(z)=\left(\frac{z}{2}\right)^{\nu} \frac{1}{2 \pi i} \int_{\epsilon-i \infty}^{\epsilon+i \infty} \frac{d t}{t^{\nu+1}} e^{\left(t+z^{2} / 4 t\right)}:=\frac{1}{2 \pi}\left(\frac{z}{4 \pi}\right)^{\nu} \hat{I}_{\nu}(z)
$$

In order to reach (2.11) from (2.10), notice however that one should extend the contour $C$ to the whole line $\epsilon+i \mathbb{R}$. While this would have lead to an infinite multiplicative factor in (2.8) (a Dirac delta at integer $N$ rather than a Kronecker delta), this is no longer a problem in (2.10), where periodicity under $\beta \rightarrow \beta+2 \pi i$ has been broken. We thus obtain

$$
p_{24}(N) \sim 2^{4} \hat{I}_{13}(4 \pi \sqrt{N-1}) .
$$

Using the asymptotic expansion of $\hat{I}_{\nu}(z)$ at large $z$ (see e.g. [53])

$\hat{I}_{\nu}(z) \sim \frac{e^{z}}{\sqrt{2}}\left(\frac{z}{4 \pi}\right)^{-\nu-\frac{1}{2}}\left[1-\frac{(\mu-1)}{8 z}+\frac{(\mu-1)\left(\mu-3^{2}\right)}{2 !(8 z)^{2}}-\frac{(\mu-1)\left(\mu-3^{2}\right)\left(\mu-5^{2}\right)}{3 !(8 z)^{3}}+\ldots\right]$,

where $\mu=4 \nu^{2}$, we can thus compute the subleading corrections to the microscopic entropy of DH states,

$$
\log \Omega(n, w) \sim 4 \pi \sqrt{|n w|}-\frac{27}{4} \log |n w|+\frac{15}{2} \log 2-\frac{675}{32 \pi \sqrt{|n w|}}-\frac{675}{2^{8} \pi^{2}|n w|}-\ldots
$$

This is however not the complete asymptotic expansion of $\Omega(n, w)$ at large charge: indeed, there are exponentially suppressed corrections to (2.12) which can be computed by using the general Rademacher expansion formula for the Fourier coefficients of modular forms with weight $w<0$ (see Appendix A). For the case at hand, we have

$$
\Omega(n, w)=2^{4} \sum_{c=1}^{\infty} c^{-14} \mathrm{Kl}(n w+1,-1 ; c) \hat{I}_{13}\left(\frac{4}{c} \pi \sqrt{|n w|}\right)
$$

where $\operatorname{Kl}(N,-1 ; c)$ are the Kloosterman sums defined in (A.7), which are uniformly bounded by $|c|$. Although each term is exponentially suppressed with respect to the previous one in the sum, they all become large at large charge. 


\subsection{Generalized Attractor Formalism for $\mathcal{N}=4$ and Leading Entropy}

Now, we would like to compute the black hole degeneracies from the macroscopic side. Since the attractor formalism is tailored for $\mathcal{N}=2$ supergravity, one should first decompose the spectrum under an $\mathcal{N}=2$ subalgebra. The $\mathcal{N}=4$ supergravity multiplet consists of the $\mathcal{N}=2$ supergravity multiplet with its graviphoton gauge field, two $\mathcal{N}=2$ gravitino multiplets with 2 Abelian gauge fields each, and one $\mathcal{N}=2$ vector multiplet. In addition, each $\mathcal{N}=4$ vector multiplet decomposes into one vector and one hypermultiplet of $\mathcal{N}=4$. The gauge fields from the $\mathcal{N}=2$ gravitino multiplets have different couplings from the rest of the $\mathcal{N}=2$ vectors and we will restrict to black holes which are neutral with respect to them. In terms of $\mathcal{N}=2$ multiplets, the spectrum of type IIA $/ K 3 \times T^{2}$ has therefore $n_{V}=24$ Abelian gauge fields. In order to evaluate the generalized prepotential $F\left(X^{A}, W^{2}\right)$ which governs the $\mathcal{N}=2$ supersymmetric couplings of these 24 gauge fields, recall the following:

i) The tree-level topological amplitude $F_{0}$ is fixed by the triple intersection product on $H^{2}\left(K^{3} \times T^{2}\right)$. We choose a basis of two-cycles with $\gamma_{1}=H^{2}\left(T^{2}\right)$ and $\gamma_{a=2,23}$ a basis of $H^{2}(K 3)$. The triple intersection product vanishes except between $\gamma_{1}$ and two 2-cycles $\gamma_{a}, \gamma_{b}$ in $H^{2}(K 3)$, where it equals the signature $(3,19)$ intersection product $C_{a b}$

ii) The topological amplitude $F_{1}$ has been computed in [41], and can be obtained as the holomorphic part of the $R^{2}$ amplitude at one-loop,

$$
f_{R^{2}}=24 \log \left(T_{2}|\eta(T)|^{4}\right)
$$

where $T, U$ denote the Kähler and complex structure moduli of the torus $T^{2}$. From the heterotic point of view, this result can be interpreted as NS5-brane instanton corrections to the tree-level heterotic $R^{2}$ amplitude [41].

iii) All higher topological amplitudes $F_{h}$ for $h>1$ vanish for models with $\mathcal{N}=4$ supersymmetry. Indeed, the type II dilaton is part of the second factor in (2.1), and a non-vanishing $F_{h}$ amplitude would be inconsistent with $S O\left(6, n_{V}-2\right)$ duality.

We therefore obtain the generalized prepotential

$$
F\left(X^{I}, W^{2}\right)=-\frac{1}{2} \sum_{a, b=2}^{23} C_{a b} \frac{X^{a} X^{b} X^{1}}{X^{0}}-\frac{W^{2}}{128 \pi i} \log \Delta(q)
$$


where $T=X^{1} / X^{0}$ and $q=e^{2 \pi i T}$. The appearance of the same discriminant function $\Delta(q)$ as in the heterotic result $(2.6)$ is at this stage coincidental ${ }^{7}$.

We may now apply the $\mathcal{N}=2$ attractor formalism summarized in Section 1.1 to the heterotic DH states $(n, w)$, or equivalently to bound states of $p^{1}=w$ D4branes wrapping $K 3$ with $q_{0}=n \mathrm{D} 0$-branes. Since this does not cause any additional complications, we shall allow arbitrary electric charges $q_{0}, q_{i=2.23}$, as long as $q_{1}=0$ and the only non-vanishing magnetic charge is $p^{1}$. Under these assumptions, the black hole free energy (1.2) reduces to

$$
\mathcal{F}\left(\phi^{I}, p^{I}\right)=-\frac{\pi}{2} C_{a b} \frac{\phi^{a} \phi^{b} p^{1}}{\phi^{0}}-\log |\Delta(q)|^{2}
$$

where

$$
q=\exp \left[\frac{2 \pi}{\phi^{0}}\left(p^{1}+i \phi^{1}\right)\right]
$$

According to (1.1), the Bekenstein-Hawking-Wald entropy is simply obtained by performing a Legendre transform over all electric potentials $\phi^{I}, I=0, \ldots 23$. The Legendre transform over $\phi^{a=2 . .23}$ sets $\phi^{a}=\left(\phi^{0} / p^{1}\right) C^{a b} q_{b}$, where $C^{a b}$ is the inverse of the matrix $C_{a b}$. We will check a posteriori that in the large charge limit, it is consistent to approximate $\Delta(q) \sim q$, whereby all dependence on $\phi^{1}$ disappears. We thus obtain

$$
S_{B H W} \sim \operatorname{Extr}_{\phi^{0}}\left[-\frac{\pi}{2} \frac{C^{a b} q_{a} q_{b}}{p^{1}} \phi^{0}+4 \pi \frac{p^{1}}{\phi^{0}}+\pi \phi^{0} q_{0}\right]
$$

The extremum of the bracket lies at

$$
\phi_{*}^{0}=\frac{1}{2} \sqrt{-p^{1} / \hat{q}_{0}}, \quad \hat{q}_{0}:=q_{0}+\frac{1}{2 p^{1}} C^{a b} q_{a} q_{b}
$$

so that at the horizon the Kähler class $\operatorname{Im} T \sim \sqrt{-p^{1} \hat{q}_{0}}$ is very large, justifying our assumption. Evaluating (2.20) at the extremum, we find

$$
S_{B H} \sim 4 \pi \sqrt{Q^{2} / 2}, \quad Q^{2}=2 p^{1} q_{0}+C^{a b} q_{a} q_{b}
$$

in agreement with the leading exponential behavior in (2.14), including the precise numerical factor. Note that this result is independent of the OSV conjecture, and relies

\footnotetext{
${ }^{7}$ The two are however related by the following chain of arguments: the $R^{2}$ coupling is related by mirror symmetry to a $\left(\nabla^{2} S\right)^{2}$ coupling, where $S$ is the type IIA axio-dilaton [20]. The latter can be computed from a 1-loop amplitude on the heterotic side, which produces both a 1-loop $\log \left(U_{2}|\eta(U)|^{4}\right)$ contribution in type IIA, and a series of D-instanton contributions on $K 3 \times S^{1}$; the latter are governed by the Fourier coefficients of $1 / \Delta(q)$, in agreement with the partition function of the $D 0-D 4$ system [54].
} 
only on the classical attractor mechanism in the presence of higher-derivative corrections. This observation, first made in [37], indicates that the tree-level $R^{2}$ coupling in the effective action of the heterotic string on $T^{6}$ (or, equivalently, large volume limit of the 1-loop $R^{2}$ coupling in type IIA $/ K 3 \times T^{2}$ ) is sufficient to cloak the singularity of the small black hole behind a smooth horizon. This is in fact confirmed by a study of the corrected geometry $[36,40]$. Furthermore, the fact that the correct numerical factor is reproduced from $R^{2}$ interactions alone indicates that, in contrast to general expectations based on the form of the tree-level metric [15], further higher-derivative interactions do not correct the Bekenstein-Hawking-Wald entropy (although they may still correct the actual solution). It would be interesting to understand the origin of this non-renormalization.

\subsection{Testing the OSV Formula}

We are now ready to test the proposal (1.7) and evaluate the inverse Laplace transform of $\exp (\mathcal{F})$ with respect to the electric potentials,

$$
\Omega_{O S V}\left(p^{I}, q^{I}\right)=\int d \phi^{0} d \phi^{1} d^{22} \phi^{a} \frac{1}{|\Delta(q)|^{2}} \exp \left[-\frac{\pi}{2} C_{a b} \frac{\phi^{a} \phi^{b} p^{1}}{\phi^{0}}+\pi \phi^{0} q_{0}+\pi \phi^{a} q_{a}\right]
$$

Due to the non-definite signature of $C_{a b}$, the integral over $\phi^{a}$ diverges for real values. This may be avoided by rotating the integration contour to $\epsilon+i \mathbb{R}$ for all $\phi$ 's. The integral over $\phi^{a}$ is now a Gaussian, leading to

$$
\Omega_{O S V}\left(p^{I}, q^{I}\right)=\int d \phi^{0} d \phi^{1}\left(\frac{\phi_{0}}{p^{1}}\right)^{11} \frac{1}{|\Delta(q)|^{2}} \exp \left(-\frac{1}{2} \frac{C^{a b} q_{a} q_{b}}{p^{1}} \phi^{0}+q_{0} \phi^{0}\right)
$$

where we dropped numerical factors and used the fact that $\operatorname{det} C=1$. Unfortunately, for imaginary $\phi^{0}, \phi^{1}, q$ is a pure phase, and $\Delta(q)$ is ill-defined. The asymptotics of $\Omega$ is independent of the details of the contour, as long as it selects the correct classical saddle point (2.21) at large charge. Approximating again $\Delta(q) \sim q$, we find the quantum version of $(2.20)$,

$$
\Omega_{O S V}\left(p^{I}, q^{I}\right)=\int d \phi^{0} d \phi^{1}\left(\frac{\phi_{0}}{p^{1}}\right)^{11} \exp \left(-\frac{1}{2} \frac{C^{a b} q_{a} q_{b}}{p^{1}} \phi^{0}-4 \pi \frac{p^{1}}{\phi^{0}}+q_{0} \phi^{0}\right)
$$

The integral over $\phi^{1}$ superficially leads to an infinite result. However, since the free energy is invariant under $\phi^{1} \rightarrow \phi^{1}+\phi^{0}$, it is natural to restrict the integration to a single period $\left[0, \phi^{0}\right]$, leading to an extra factor of $\phi^{0}$ in $(2.25)$. The integral over $\phi^{0}$ is now of Bessel type, leading to

$$
\Omega_{O S V}\left(p^{I}, q^{I}\right)=\left(p^{1}\right)^{2} \hat{I}_{13}\left(4 \pi \sqrt{Q^{2} / 2}\right)
$$


in impressive agreement with the microscopic result (2.12) at all orders in $1 / Q$.

While this result is encouraging, it however indicates that (1.7) should interpreted with some care:

- The extra factor of $\left(p^{1}\right)^{2}$ in Equation (2.26) is inconsistent with $O\left(\Gamma_{6,22}\right)$ duality, which requires the exact degeneracies to be a function of $Q^{2}$ only. This indicates that the integration measure implicit in (1.7) is not the trivial Euclidean measure. Given the wave function interpretation of $e^{F_{t o p}}$ [55], one attractive possibility would be to normalize it - alas, it appears to be severely non-normalizable. For lack of a proper understanding of this integration measure, we are thus forced to consider ratios $\Omega(p, q) / \Omega\left(p, q^{\prime}\right)$ only $^{8}$.

- In order to obtain the modified Bessel function with the correct index, note that it was crucial to discard the non-holomorphic correction proportional to $\log T_{2}$ in $F_{1}$ (keeping this correction would have resulted in an index 19 rather than 13, spoiling the agreement with the microscopic result (2.12)). In addition, it was important to compare to the degeneracies of DH states with arbitrary angular momentum $J$ (degeneracies of DH states with $J=0$ are computed in Appendix $\mathrm{C}$, and lead to a Bessel function with index 29/2 and a different intercept).

- The "all order" result (2.26) depends only on the number of $\mathcal{N}=2$ vector multiplets, as well as on the leading large volume behavior of $F_{1} \sim q /(128 \pi i)$. By heterotic/type II duality, this term is mapped to a tree-level $R^{2}$ interaction on the heterotic side, which is in fact universal. We thus conclude that in all $\mathcal{N}=2$ models which admit a dual heterotic description, the degeneracies of small black holes predicted by (1.7) are given by

$$
\Omega_{O S V}\left(p^{I}, q^{I}\right) \propto \hat{I}_{\frac{n_{V}+2}{2}}\left(4 \pi \sqrt{Q^{2} / 2}\right),
$$

provided it is justified to neglect higher genus $F_{h>1}$ and genus 0,1 Gromov-Witten instantons. We shall return to this point in Section 4.3.

- In order to try and match (2.26) and (2.12) in more detail, one may change variable $\beta=\pi / t$ in (2.8) and rewrite the exact microscopic result as

$$
\Omega(n, w)=\int d t t^{-14} \frac{\exp \left(\frac{\pi n w}{t}\right)}{\Delta\left(e^{-4 \pi t}\right)}
$$

${ }^{8}$ The analysis of the $p^{0} \neq 0$ case in Section 4.4 indicates that a proper duality-covariant measure will have to break holomorphicity. 
On the other hand, it is convenient to change variables in the OSV integral (2.24) to $\tau_{1}=\phi^{1} / \phi^{0}, \tau_{2}=-p^{1} / \phi^{0}$, with Jacobian $d \phi^{0} d \phi^{1}=8\left(p^{1}\right)^{2} d \tau_{1} d \tau_{2} / \tau_{2}^{3}$, leading to

$$
\Omega_{O S V}\left(p^{I}, q^{I}\right) \sim \int d \tau_{1} d \tau_{2} \tau_{2}^{-14} \frac{\exp \left(\frac{\pi(N-1)}{\tau_{2}}\right)}{\left|\Delta\left(e^{-2 \pi \tau_{2}+2 \pi i \tau_{1}}\right)\right|^{2}}
$$

Despite obvious similarities, it appears unlikely that the two results are equal nonperturbatively. Indeed, with any natural interpretation of the integration contours consistent with the quantum mechanics interpretation, the integral (2.29) diverges.

- Just as the perturbative result (2.12), the result (2.26) misses subleading terms in the Rademacher expansion (2.15). It does not seem possible to interpret any of the terms with $c>1$ as the contribution of a subleading saddle point in either (2.10) or (2.24). It would be interesting to see if non-holomorphic Poincaré series can be used to extract these contributions from (2.24).

Despite these difficulties, we find it remarkable that the black hole partition function in the OSV ensemble, obtained from purely macroscopic considerations, reproduces the entire asymptotic series exactly to all orders in inverse charge.

\subsection{Degeneracies vs. Helicity Supertrace}

If it is to satisfy the second law of thermodynamics, the Bekenstein-Hawking-Wald entropy should be equal to the logarithm of the total number of micro-states in the regime where the black hole is formed. On the other hand, the degeneracies of DH states have been computed at zero heterotic string coupling. In general however, BPS states can appear and disappear rather chaotically on various loci of the moduli space, by (un)pairing up into longer multiplets. If the absolute degeneracies at zero coupling can be identified with a suitable index, it is then possible to ensure that the total number of micro-states does not change as the coupling is increased (barring the possible crossing of lines of marginal stability). The only such indices with a well-defined target space interpretation are the helicity supertraces ${ }^{9}$

$$
\Omega_{n}=\operatorname{Tr}(-1)^{F} J_{3}^{n}
$$

where $F$ is the target-space fermion number and $J_{3}$ is a Cartan generator in the massive little group in $3+1$ dimensions (or, for massless states, the ordinary helicity), and $n$ is an even number $\left(\Omega_{2 n+1}\right.$ always vanishes by reason of symmetry). For a given

\footnotetext{
${ }^{9}$ see [56], Appendices E and G for an extensive review of helicity supertraces.
} 
number $\mathcal{N}$ of supersymmetry in 4 dimensions, $\Omega_{n<\mathcal{N}}$ vanishes automatically in any multiplet, while $\Omega_{n \geq 2 \mathcal{N}}$ receives contributions from generic long multiplets. In the window $\mathcal{N} \leq n \leq 2 \mathcal{N}$, the helicity supertraces $\Omega_{n}$ receive only contributions from short or intermediate multiplets, and are therefore unaffected by recombination processes.

For the $\mathcal{N}=4$ case of interest in this section, the first non-vanishing supertrace is $\Omega_{4}$, which receives contributions only from the supergravity multiplet, massless vector multiplet and short massive multiplets ${ }^{10}$,

$$
\Omega_{4}(\text { sugra })=3, \quad \Omega_{4}(\text { vect })=\frac{3}{2}, \quad \Omega_{4}\left(S^{j}\right)=\frac{3}{2}(2 j+1)(-1)^{2 j}
$$

while the intermediate and long $\mathcal{N}=4$ multiplets cancel out. In particular, $\Omega_{4}$ is unaffected by possible recombinations of four short multiplets into a longer intermediate multiplet. Similarly, the helicity supertrace $\Omega_{6}$ receives contributions from short and intermediate multiplets only,

$$
\begin{gathered}
\Omega_{6}(\text { sugra })=\frac{13 \cdot 15}{4}, \quad \Omega_{6}(\text { vect })=\frac{15}{8}, \quad \Omega_{6}\left(S^{j}\right)=\frac{15}{8}(2 j+1)^{3}(-1)^{2 j}, \\
\Omega_{6}\left(I^{j}\right)=\frac{45}{4}(2 j+1)(-1)^{2 j+1}
\end{gathered}
$$

and is invariant under recombinations of four intermediate multiplets into a longer one.

In order to compare with the absolute degeneracies (2.6), let us compute the helicity supertrace of the DH states in the Het $(4,24)$ model. Helicity supertraces are most easily computed by introducing generating parameters $v$ and $\bar{v}$ for the left and right moving components of the space-time helicity $J_{3}[56]$

$$
Z(v, \bar{v})=\operatorname{Tr}(-1)^{F} e^{2 \pi i v J_{3}^{R}} e^{2 \pi i \bar{v} J_{3}^{L}} q^{L_{0}} q^{\bar{L}_{0}}
$$

and computing

$$
B_{n}(q, \bar{q})=\sum \Omega_{n} q^{L_{0}} \bar{q}^{\bar{L}_{0}}=\left.\left(\frac{\partial}{2 \pi i \partial v}+\frac{\partial}{2 \pi i \partial \bar{v}}\right)^{n}\right|_{v=\bar{v}=0} Z(v, \bar{v})
$$

The generating function for helicity supertraces of the $E_{8} \times E_{8}$ heterotic string on $T^{6}$ is simply given by

$$
Z_{(4,24)}^{H}(v, \bar{v})=\frac{\xi(v) \bar{\xi}(\bar{v})}{\tau_{2}|\eta|^{4}} \frac{1}{2} \sum_{\alpha, \beta}(-1)^{\alpha+\beta+\alpha \beta} \frac{\bar{\theta}\left[\begin{array}{c}
\alpha / 2 \\
\beta / 2
\end{array}\right](\bar{v}) \bar{\theta}^{3}\left[\begin{array}{c}
\alpha / 2 \\
\beta / 2
\end{array}\right]}{\bar{\eta}^{4}} \frac{Z_{6,6}}{|\eta|^{12}}\left(\theta_{E_{8}[1]}\right)^{2}
$$

\footnotetext{
${ }^{10}$ The superscript $j$ indicates the spin $J_{3}$ of the middle state in the short massive supermultiplet $S^{j}$
} 
where $\alpha, \beta=0,1$ label the four spin structures on the superconformal side, $\xi(v)$ incorporates the $U(1)$ charge of the bosons in the two transverse directions,

$$
\xi(v)=\prod_{n=1}^{\infty} \frac{\left(1-q^{n}\right)^{2}}{\left(1-q^{n} e^{2 \pi i v}\right)\left(1-q^{n} e^{-2 \pi i v}\right)}=\frac{2 \eta^{3} \sin \pi v}{\theta_{1}(v)}
$$

$\theta_{E_{8}[1]}$ is the numerator of the character of the $E_{8}$ current algebra at level 1,

$$
\theta_{E_{8}[1]}=\frac{1}{2}\left(\theta_{3}^{8}+\theta_{4}^{8}+\theta_{2}^{8}\right)
$$

and $Z_{6,6}$ is the partition function of bosonic zero-modes on $T^{6}$. By the Riemann identity, (2.36) can be converted into

$$
Z_{(4,24)}^{H}(v, \bar{v})=\frac{\xi(v) \bar{\xi}(\bar{v})}{\tau_{2}|\eta|^{4}} \frac{\bar{\theta}^{4}\left[\begin{array}{c}
1 / 2 \\
1 / 2
\end{array}\right](\bar{v} / 2)}{\bar{\eta}^{4}} \frac{Z_{6,6}}{|\eta|^{12}}\left(\theta_{E_{8}[1]}\right)^{2}
$$

which is recognized as a trace in the Ramond sector only, with an insertion of $(-1)^{J_{R}}$. Since the Jacobi theta function $\theta_{1}(z ; \tau)$ has a single zero at $z=0$, a non-vanishing supertrace is obtained only for $n \geq 4$. Taking four $\bar{v}$-derivatives and using $\theta\left[\begin{array}{l}1 / 2 \\ 1 / 2\end{array}\right]^{\prime}(0)=$ $\theta_{1}^{\prime}(0)=2 \pi \eta^{3}, \xi(0)=1$, the first non-vanishing supertrace is easily computed:

$$
B_{4}=\frac{1}{\tau_{2}} Z_{6,6}\left(\theta_{E_{8}[1]}\right)^{2} \times \frac{3}{2} \frac{1}{\eta^{24}}
$$

where the factor $1 / \tau_{2}$ corresponds to the contribution of the zero-mode $p_{2}, p_{3}$ in the transverse directions. At a generic point, the two factors in the numerator combine into a lattice sum $Z_{(6,22)}$, leading to

$$
B_{4}=\frac{1}{\tau_{2}} Z_{6,22} \times \frac{3}{2} \frac{1}{\eta^{24}}
$$

The first factor simply corresponds to the continuous degeneracy due to the momentum in 4 dimensions, while the second factor is just the partition function of the lattice $\Gamma_{6,22}$ of electric charges. For any vector $Q \in \Gamma_{6,22}$, we conclude that the helicity supertrace of states with electric charges $Q$ is given by

$$
\Omega_{4}(Q)=\frac{3}{2} p_{24}(N)=\frac{3}{32} \Omega_{a b s}(Q)
$$

where $\Omega_{a b s}$ is the absolute degeneracy computed in (2.6) up to an overall numerical factor. This suggests that, in the case of $\mathcal{N}=4$ backgrounds, the OSV integral (1.7) may compute the fourth helicity supertrace of the black hole micro-states. 
An immediate problem with this proposal is that it implies that the OSV prescription should vanish in the case of "large" black holes, which form intermediate (1/4-BPS) multiplets of $\mathcal{N}=4$ supersymmetry. These states cancel from $\Omega_{4}$ and contribute to sixth helicity supertrace $\Omega_{6}$ onward. In the case of the $\operatorname{Het}(4,24)$ model, $\Omega_{6}$ may be obtained straightforwardly by taking either $6 \bar{v}$-derivatives, or $4 \bar{v}$-derivatives and 2 $v$-derivatives, leading to [56]

$$
B_{6}=\frac{1}{\tau_{2}} Z_{6,22} \times \frac{15}{8} \frac{2-E_{2}}{\eta^{24}}
$$

Since the perturbative heterotic spectrum contains no intermediate multiplets, this result arises from the contributions of the same DH states which contributed to (2.41). While the Rademacher formula does not apply to the non-modular invariant Eisenstein series $E_{2}$, one may simply use the identity

$$
\frac{E_{2}}{\eta^{24}}=-q \frac{d}{d q} \frac{1}{\eta^{24}}
$$

to obtain the asymptotic behavior of the Fourier coefficients of $B_{6}$ to all orders in $1 / N$,

$$
\Omega_{6}(N) \sim \frac{15}{8}(N+1) \hat{I}_{13}(4 \pi \sqrt{N-1})
$$

where

$$
B_{6}=\frac{1}{\tau_{2}} Z_{6,22} \sum_{N=0}^{\infty} \Omega_{6}(N) q^{N-1}
$$

In particular, the extra factor of $N+1$ in (2.45) makes it impossible to include a contribution from $\Omega_{6}$ to the index relevant for the OSV proposal (1.7) for half-BPS states, since one would have to modify the integration measure by a $q$ dependent factor. On the other hand, $\Omega_{4}$ is clearly inadequate for $1 / 4$ BPS states. We conclude that the index computed by (1.7) must depend on the number of supersymmetries preserved by the BPS states under consideration.

Before closing this section, let us briefly comment on the case with $\mathcal{N}=2$ supersymmetry originally envisaged in [14]. In this case, the only index protected by supersymmetry is the second helicity supertrace $\Omega_{2}$, to which only $1 / 2$ BPS states contribute:

$$
\left.\Omega_{2}(\text { sugra })=\Omega_{2}(\text { vect })=1, \quad \Omega_{2} \text { (hyper }\right)=-1, \quad \Omega_{2}\left(S^{j}\right)=(2 j+1)(-1)^{2 j+1}
$$

This is the space-time interpretation of the "vectors minus hypers" index introduced from a world-sheet point of view in [57], since short multiplets with integer (resp. halfinteger) spin $j$ are the massive generalization of the massless hypermultiplet (resp. 
vector multiplet). In particular, $\Omega_{2}$ is invariant under the recombination of a hyper and a vector multiplet into a long multiplet of $\mathcal{N}=2$. Note however that $\Omega_{2}$ may change at lines of marginal stability in moduli space. Since we do not have the freedom to add higher helicity supertraces, we conjecture that the OSV prescription computes the second helicity supertrace of the $\mathcal{N}=2$ black hole micro-states. Evidence for this claim will be given in Section 5 .

\section{$2.7 \mathrm{DH}$ states in type $\mathrm{II} / K 3 \times T^{2}$}

In addition to the heterotic DH states, the $(4,24)$ model also admits DH states on the type IIA side, corresponding to fundamental type II strings with momentum $n_{i}$ and winding $w^{i}$ along $T^{2}(i=5,6)$. These can have either left-moving or right-moving excitations, depending on the sign of $n_{i} w^{i}$. Since there are now 8 bosonic and 8 fermionic oscillators, with total central charge $c=12$, the degeneracies grow as

$$
S_{D H}^{I I A} \sim 2 \pi \sqrt{2\left|n_{i} w^{i}\right|}
$$

In contrast to the heterotic DH states, these states preserve only $1 / 4$ of the supersymmetries, unless $n_{i} w^{i}=0$. According to (2.4), they have $P^{2}=Q^{2}=P \cdot Q=0$, hence zero tree-level entropy. Their helicity supertraces have been computed in [56] (eqs. $(G .24)$ and $(G .25))$, and vanish identically except for $n_{i} w^{i}=0$ :

$$
\begin{aligned}
& \Omega_{4}(Q)=36 \delta_{n_{i} w^{i}, 0} \\
& \Omega_{6}(Q)=90 \delta_{n_{i} w^{i}, 0}
\end{aligned}
$$

This indicates that these intermediate multiplets come in pairs and may combine into longer multiplets and leave the spectrum.

Since the type II DH states are charged under the four $\mathcal{N}=2$ gravitino multiplets, the $\mathcal{N}=2$ attractor formalism does not apply directly. Nevertheless, by a $O(6,22)$ duality, they may be mapped to a D0-D2/T $T^{2}$ state with charges $\left(q_{0}, q_{1}\right)$.

More generally, we may try and apply the OSV formula (1.7) to purely electrically charged states in the type II polarization, with arbitrary electric charges $\left(q_{0}, q_{1}, q_{a}\right)$. The perturbative part of the free-energy (1.2) vanishes, leaving only the Gromov-Witten instanton series, evaluated at real $X^{A}=\phi^{A} / \phi^{0}$, where it is no longer convergent. The integral (1.7) is therefore highly singular. Nevertheless, discarding the Gromov-Witten contribution, (1.7) produces a delta function of the electric charges, in qualitative agreement with the helicity supertraces above.

It should be noticed that similar DH states occur in Type IIA $/ T^{6}$, with $\mathcal{N}=8$ supersymmetry. The first non-trivial helicity supertraces occur at order $\Omega_{12}, \Omega_{14}$, but they are given by modular forms with positive weight, so that the indexed degeneracies of intermediate multiplets grow as a power-law rather than exponentially. 


\section{Small Black Holes in $\mathcal{N}=4$ Models with Reduced Rank}

In this section, we proceed to compare the macroscopic and microscopic entropy of small black holes in a variety of string vacua with $\mathcal{N}=4$ supersymmetry. While the $(4,24)$ model discussed in the previous section has been the most studied one in the literature, a large number of $\mathcal{N}=4$ vacua can be obtained using fermionic [58,59] or orbifold constructions [60-62]. The latter has the advantage that a dual description can often be found by using six-dimensional heterotic/type II duality and adiabatic arguments $[61,63,64]$. Each of these models has a moduli space of the form (2.1), where the first factor corresponds to the heterotic dilaton and $n_{V}$ denotes the number of massless Abelian gauge fields (including the graviphoton, but discarding the gauge fields from the two $\mathcal{N}=2$ gravitino multiplets). We will denote such vacua as $\operatorname{Het}\left(4, n_{V}\right)$ or $I I\left(4, n_{V}\right)$, assuming that all models with the same number of vector multiplets belong to the same moduli space. As in the $(4,24)$ case, the only non-vanishing F-term $F_{1}$ can be extracted from the one-loop amplitude $R^{2}$ amplitude in the type II model, while the exact degeneracies of small black holes are most easily determined in the heterotic dual.

\section{1 $F_{1}$ in Reduced Rank Type II Models}

The topological amplitude $F_{1}$ has been computed in a number of $\left(4, n_{V}\right)$ type II models in [61]. In general, it is given by the holomorphic, $T$-dependent part of the integral of the "new" supersymmetric index on the fundamental domain of the upper half plane [41],

$$
f_{R^{2}}=\int_{\mathcal{F}} \frac{d^{2} \tau}{\tau_{2}} \operatorname{Tr}_{R R}(-1)^{J_{L}+J_{R}} J_{L} J_{R} q^{L_{0}+\bar{L}_{0}}=-\frac{2}{3} \int_{\mathcal{F}} \frac{d^{2} \tau}{\tau_{2}} B_{4}
$$

As indicated in the second equality, the supersymmetric index is proportional to the generating function $B_{4}$ of the helicity supertraces $\Omega_{4}$ of the perturbative type II spectrum [61]. For completeness, we briefly review the CFT construction of these models ${ }^{11}$ and list the corresponding supertrace and $R^{2}$ amplitudes:

- The $(4,16)$ model is obtained by starting from Type IIA on $K 3 \times T^{2}$ at the $T^{4} / \mathbb{Z}_{2}$ orbifold point of $K 3$, and performing a further $Z_{2}$ orbifold which acts as $(-1)$ on half of the twisted sectors, and shifts one of the coordinates of $T^{2}$ by a half-period. The generating function of the 4-th helicity supertraces is

$$
B_{4}=18 Z_{2,2}+6 \sum_{(h, g) \neq(0,0)} Z_{2,2}^{\delta_{1}}\left[\begin{array}{l}
h / 2 \\
g / 2
\end{array}\right]
$$

\footnotetext{
${ }^{11}$ While the inclusion of discrete $\mathrm{RR}$ fluxes on $K 3$ is required non-perturbatively for level matching [63], this does not affect the perturbative computation of $F_{1}$ in these models. Such fluxes do however affect the BPS spectrum [65].
} 
where

$$
Z_{2,2}^{\delta}\left[\begin{array}{l}
h / 2 \\
g / 2
\end{array}\right](T, U ; \tau, \bar{\tau})=\sum_{p \in \Gamma_{2,2}+\frac{h}{2} \delta} e^{-i \pi g(p, \delta)} q^{\frac{1}{2} \Pi_{L}^{2}(p)} \bar{q}^{\frac{1}{2} \Pi_{R}^{2}(p)}
$$

is the shifted lattice sum for the Narain lattice of the torus $T^{2}$. We choose a symmetric shift vector $\delta_{1}=(1,1) / 2$ along the first circle, so as to entertain a geometric description.

- A $(4,12)$ model may be obtained by performing a further $\mathbb{Z}_{2}$ orbifold of the $(4,16)$ model, which acts as $(-1)$ on a different half of the 16 twisted states, together with a shift by half a period on the remaining circle in $T^{2}$. The helicity supertrace generating function is

$$
B_{4}=9 Z_{2,2}+3 \sum_{(h, g) \neq(0,0)}\left(Z_{2,2}^{\delta_{1}}\left[\begin{array}{l}
h / 2 \\
g / 2
\end{array}\right]+Z_{2,2}^{\delta_{2}}\left[\begin{array}{l}
h / 2 \\
g / 2
\end{array}\right]+Z_{2,2}^{\delta_{1}+\delta_{2}}\left[\begin{array}{l}
h / 2 \\
g / 2
\end{array}\right]\right)
$$

- A $(4,8)$ model can be obtained by returning to the $I I(4,24)$ model at the $T^{4} / \mathbb{Z}_{2}$ orbifold point, and by orbifolding by a further $\mathbb{Z}_{2}$ which acts as $(-1)$ on all twisted sectors. The result is

$$
B_{4}=18 Z_{2,2}-6 \sum_{(h, g) \neq(0,0)} Z_{2,2}^{\delta}\left[\begin{array}{l}
h / 2 \\
g / 2
\end{array}\right]
$$

In each of these cases, the modular integral $(3.1)$ can be reduced to the $(4,24)$ case by making use of the following identities,

$$
\begin{aligned}
& \frac{1}{2}\left(Z_{2,2}\left[\begin{array}{l}
00 \\
00
\end{array}\right]+Z_{2,2}\left[\begin{array}{c}
00 \\
1 \frac{1}{2} 0
\end{array}\right]+Z_{2,2}\left[\begin{array}{c}
\frac{1}{2} 0 \\
00
\end{array}\right]+Z_{2,2}\left[\begin{array}{c}
\frac{1}{2} 0 \\
\frac{1}{2} 0
\end{array}\right]\right)=Z_{2,2}(T / 2,2 U) \\
& \frac{1}{2}\left(Z_{2,2}\left[\begin{array}{l}
00 \\
00
\end{array}\right]+Z_{2,2}\left[\begin{array}{c}
00 \\
0 \frac{1}{2}
\end{array}\right]+Z_{2,2}\left[\begin{array}{c}
0 \frac{1}{2} \\
00
\end{array}\right]+Z_{2,2}\left[\begin{array}{c}
0 \frac{1}{2} \\
0 \frac{1}{2}
\end{array}\right]\right)=Z_{2,2}(T / 2, U / 2) \\
& \frac{1}{2}\left(Z_{2,2}\left[\begin{array}{l}
00 \\
00
\end{array}\right]+Z_{2,2}\left[\begin{array}{l}
00 \\
\frac{1}{2} \frac{1}{2}
\end{array}\right]+Z_{2,2}\left[\begin{array}{l}
\frac{1}{2} \frac{1}{2} \\
00
\end{array}\right]+Z_{2,2}\left[\begin{array}{c}
\frac{1}{2} \frac{1}{2} \\
\frac{1}{2} \frac{1}{2}
\end{array}\right]\right)=Z_{2,2}(T / 2,(U+1) / 2),
\end{aligned}
$$

where, on the left hand side, all partition functions are evaluated at $(T, U)$. We thus obtain [61]

$$
\begin{aligned}
(4,24): f_{R^{2}}=24 \log T_{2}\left|\eta(T)^{4}\right| & \sim 24 \log T_{2}-8 \pi T_{2}+\ldots \\
(4,16): f_{R^{2}}=16 \log T_{2}\left|\eta^{3}(T) \theta_{4}(T)\right| & \sim 16 \log T_{2}-4 \pi T_{2}+\ldots \\
(4,12): f_{R^{2}}=12 \log T_{2}\left|\eta^{2}(T) \theta_{4}^{2}(T)\right| & \sim 12 \log T_{2}-2 \pi T_{2}+\ldots \\
(4,8): f_{R^{2}}=8 \log T_{2}\left|\eta(T)^{6} / \theta_{4}(T)^{2}\right| & \sim 8 \log T_{2}-4 \pi T_{2}+\ldots
\end{aligned}
$$

where $T$ is the Kähler modulus of the $T^{2}$ covering of the base of the $K 3$ fibration. We have also indicated the large volume expansion. The leading linear term is proportional 
to the size $A$ of the base of the $K 3$ fibration, which differs from $T$ by a power of two. The logarithmic divergence is proportional to the helicity supertrace $\Omega_{4}=3+(3 / 2)\left(n_{V}-2\right)$ of the massless spectrum. The dots correspond to a finite term, dependent on the details of the IR cut-off, and a sum of worldsheet instantons. In general, we therefore have

$$
f_{R^{2}}=n_{V} \log A-8 \pi A_{2}+\ldots
$$

with $A=(T, T / 2, T / 4, T / 2)$ for the four models above. In the heterotic dual, $A$ becomes the heterotic dilaton $S=\theta+i V_{6} / g_{s}^{2}$. where $V_{6}$ is the volume of the 6 -torus. The term linear in $T_{2}$ is therefore a tree-level term, coming from the compactification of the $R^{2}$ interaction in the 10-dimensional heterotic string. The type II worldsheet instantons are interpreted on the heterotic side as Euclidean NS5-branes wrapping $T^{6}$.

\subsection{Heterotic Duals and Exact Counting of DH States}

Heterotic $\mathcal{N}=4$ models with reduced rank can be obtained by orbifolding the $E_{8} \times E_{8}$ or $S O(32)$ ten-dimensional heterotic strings at an enhanced symmetry point, by a symmetry leaving the right-moving superconformal algebra untouched. In particular, we consider the following models:

- A $(4,16)$ model obtained by orbifolding the $E_{8} \times E_{8}$ heterotic string on $T^{6}$ by the exchange of the two $E_{8}$, combined with a translation on one of the directions of the torus $T^{6}$. Equivalently, one may orbifold the $S O(32)$ heterotic string at an $S O(16) \times S O(16)$ point by the exchange of the two $S O(16)$ factors.

- A $(4,12)$ model obtained by orbifolding the $S O(32)$ heterotic string at a $S O(8)^{4}$ point by the group $\mathbb{Z}_{4}$ permuting the four $S O(8)$ factors circularly ${ }^{12}$ combined with a translation of order 4 on the torus.

- A $(4,10)$ model obtained by orbifolding the $S O(32)$ heterotic string at a $S O(4)^{8}$ point by the group $\mathbb{Z}_{8}$ permuting the eight $S O(4)$ factors circularly. Viewing $S O(4)^{8}$ as $S U(2)^{16}$, one may also orbifold by $\mathbb{Z}_{16}$ and get a $(4,9)$ model.

In each of these models, it is important to include a translation on one of the directions of the torus $T^{6}$ so as to give a mass to the twisted sectors, and ensure that the rank of the gauge group is effectively reduced.

The common property of these models is that they give rise to an enhanced gauge symmetry with a current algebra at level $k>1$. However, in order to have a type II dual with a smooth geometry, one should further break the gauge symmetry to an Abelian group $U(1)^{n_{V}+4}$.

\footnotetext{
${ }^{12}$ It is also possible to orbifold by the full permutation group $S_{4}$, or the alternate subgroup $A_{4}$, but the required action on $T^{2}$ is more complicated.
} 


\subsection{A Detailed Analysis of the Het $(4,16)$ Model}

Let us now discuss in detail the degeneracies of $\mathrm{DH}$ states in the Het $(4,16)$ model obtained by orbifolding the Het $(4,24)$ model at a point of enhanced gauge symmetry $E_{8} \times E_{8}$. The Narain lattice of the Het $(4,24)$ model may be decomposed as

$$
\Gamma_{6,22}=E_{8}(-1) \oplus E_{8}(-1) \oplus I I^{1,1} \oplus I I^{5,5}
$$

where $I I^{1,1} \oplus I I^{5,5}$ describe the momenta and winding numbers on the 6-torus $S^{1} \times T^{5}$. Accordingly, we shall denote the momentum eigenstates as $P=\left(P_{1}, P_{2}, P_{3}, P_{4}\right)$. At any point in the moduli space (2.1), this vector may be projected into a sum of a left-moving and a right-moving part,

$$
P=\Pi_{L}(P)+\Pi_{R}(P)
$$

where $\Pi_{R}(P) \in \mathbb{R}^{6}$ are the 6 central charges of $\mathcal{N}=4$ supersymmetry, and $\Pi_{L}(P) \in \mathbb{R}^{22}$ are the 22 electric charges under the vector multiplets of the $\operatorname{Het}(4,24)$ model. While the charge vector $P$ takes quantized values independent of the moduli, the projections $\Pi_{L}(P)$ and $\Pi_{R}(P)$ are real numbers depending continuously on the moduli.

\section{Untwisted sector}

Now, the Het $(4,16)$ model can be obtained as a $\mathbb{Z}_{2}$ orbifold acting on momentum eigenstates as

$$
g\left|P_{1}, P_{2}, P_{3}, P_{4}\right\rangle=e^{2 \pi i \delta \cdot P_{3}}\left|P_{2}, P_{1}, P_{3}, P_{4}\right\rangle
$$

where $2 \delta$ is the vector $(1,1) \in I I^{1,1}$ corresponding to the translation by half a period along the circle. The action on the oscillators is most easily described by diagonalizing the action of $g$ : 8 left-moving oscillators obtain a negative parity under $g$, while the remaining left-moving and all right-moving oscillators have positive parity. Let $\mathcal{P}^{ \pm}(\alpha)$ denote a generic monomial in left-moving creation oscillators, with definite parity \pm under $g$.

DH states in the untwisted sector of the Het $(4,16)$ model can be constructed as invariant combinations of the DH states of the Het $(4,24)$ model under the orbifold action,

$$
\mathcal{P}^{ \pm}(\alpha)\left(\left|P_{1}, P_{2}, P_{3}, P_{4}\right\rangle \pm e^{2 \pi i \delta \cdot P_{3}}\left|P_{2}, P_{1}, P_{3}, P_{4}\right\rangle\right) \otimes|\tilde{s}\rangle
$$

where the parity of the oscillators is correlated with that of the zero-modes, and $|\tilde{s}\rangle$ is a right-moving groundstate ${ }^{13}$. The level matching conditions identifies the level $N$ of

\footnotetext{
${ }^{13}$ In the following, we omit the factor of $2^{4}$ due to the degeneracy of the right-moving groundstate.
} 
the oscillator state $\mathcal{P}(\alpha)$ with

$$
N-1=\frac{1}{2} q_{R}^{2}-\frac{1}{2} q_{L}^{2}=\frac{1}{2} P^{2}
$$

The DH states (3.14) are thus enumerated by the partition functions

$$
\frac{1}{2}\left(\frac{1}{\eta^{24}} \pm \frac{2^{4}}{\eta^{12} \vartheta_{2}^{4}}\right) \sum_{I I^{22,6}} q^{\frac{1}{2} q_{L}^{2}} \bar{q}^{\frac{1}{2}} q_{R}^{2} \frac{1 \pm \Theta(P)}{2}
$$

where $\Theta(P)=e^{2 \pi i \delta P_{3}} \delta_{P_{1}, P_{2}}$. The last factor in (3.16) guarantees that states with $P_{1} \neq$ $P_{2}$ are counted twice with $1 / 2$ multiplicity, while states with $P_{1}=P_{2}$ and $e^{2 \pi i \delta P_{3}}=\mp 1$ are dropped out, in agreement with Equation (3.14).

This is not the final answer however, since we need to extract from (3.16) the contribution of states with a given electric charge. Due to the orbifold projection, the only massless vector multiplets are the linear combinations of the $E_{8} \times E_{8}$ gauge bosons of the $(4,24)$ model which are symmetric under exchange of the two factors. Therefore, a state of the form $\mathcal{P}(\alpha)\left|P_{1}, P_{2}, P_{3}, P_{4}\right\rangle$ has electric charge

$$
Q(P):=\left(P_{1}+P_{2} ; P_{3}, P_{4}\right)
$$

taking values in the (non self-dual) lattice ${ }^{14}$

$$
M_{0}=E_{8}\left(-\frac{1}{2}\right) \oplus I I^{1,1} \oplus I I^{5,5}
$$

In particular, the momentum eigenstates $\left(P_{1}-Q, P_{2}+Q, P_{3}, P_{4}\right)$ have the same electric charge $Q(P)$ as $\left(P_{1}, P_{2}, P_{3}, P_{4}\right)$, for any $Q$ in the $E_{8}$ root lattice. It can be checked that all these states have the same central charges $\Pi_{R}$ on the subspace $S O(6,14) / S O(6) \times$ $S O(14)$ of the moduli space of the $\operatorname{Het}(4,24)$ model $(2.1)$ invariant under the orbifold projections. They therefore have the same mass and electric charge, but differ by the excitation level $N$ of the oscillators.

In order to extract the exact degeneracy of DH states for a given electric charge $Q$, it is appropriate to change the basis and decompose the two $E_{8}(-1)$ charge vectors into their sum and difference,

$$
\begin{aligned}
& P_{1}+P_{2}=2 \Sigma+\mathcal{P} \\
& P_{1}-P_{2}=2 \Delta-\mathcal{P}
\end{aligned}
$$

\footnotetext{
${ }^{14}$ In this expression, $(-1 / 2)$ indicates that the norm of the $E_{8}$ is multiplied by $1 / 2$, in order to keep the canonical normalization for the gauge fields.
} 
where $\Sigma, \Delta$ both take values in the $E_{8}$ root lattice, and $\mathcal{P}$ is an element of the finite group $Z=\Lambda_{r}\left(E_{8}\right) / 2 \Lambda_{r}\left(E_{8}\right)$, of index $2^{8}$. Expressing the square of the left-moving momentum as

$$
\Pi_{L}^{2}\left(\Sigma+\Delta, \Sigma-\Delta+\mathcal{P}, P_{3}, P_{4}\right)=\Pi_{L}^{2}\left(\Sigma+\frac{1}{2} \mathcal{P}, \Sigma+\frac{1}{2} \mathcal{P}, P_{3}, P_{4}\right)+2\left(\Delta-\frac{1}{2} \mathcal{P}\right)^{2}
$$

we can carry out the sum over the "unphysical charges" $\Delta$ by introducing $E_{8}$ theta functions with characteristics:

$$
\Theta_{E_{8}[2], \mathcal{P}}(\tau):=\sum_{\Delta \in E_{8}(1)} e^{2 \pi i \tau\left(\Delta-\frac{1}{2} \mathcal{P}\right)^{2}}
$$

This allows to decompose the $E_{8}(1) \oplus E_{8}(1)$ lattice as a sum of products of shifted $E_{8}(2)$ lattices,

$$
\theta_{E_{8}[1]}^{2}(\tau)=\sum_{\mathcal{P} \in E_{8} / 2 E_{8}} \theta_{E_{8}[2], \mathcal{P}}(\tau) \theta_{E_{8}[2], \mathcal{P}}(\tau)
$$

Note that $\Theta_{E_{8}[2], \mathcal{P}}$ depends only on the orbit of $\mathcal{P}$ under the Weyl group of $E_{8}$. It may be checked that the finite group $Z$ decomposes into three orbits only, corresponding to the orbit of the fundamental weight of the trivial, adjoint and 3875 representations, of respective length 1, 120 and 135, respectively. The theta series (3.22) are thus the numerator of affine characters of $E_{8}$ at level 2, and can be computed explicitly using free fermion representations,

$$
\begin{aligned}
& \theta_{E_{8}[2], 1}=\theta_{E_{8}[1]}(2 \tau)=2^{-4}\left(\theta_{3}^{8}+\theta_{4}^{8}+14 \theta_{3}^{4} \theta_{4}^{4}\right) \\
& \theta_{E_{8}[2], 248}=\frac{1}{2}\left(\theta_{3}^{6} \theta_{2}^{2}+\theta_{2}^{6} \theta_{3}^{2}\right)(2 \tau)=2^{-4}\left(\theta_{3}^{8}-\theta_{4}^{8}\right) \\
& \theta_{E_{8}[2], 3875}=\theta_{3}^{4} \theta_{2}^{4}(2 \tau)=2^{-4} \theta_{2}^{8}
\end{aligned}
$$

where we used the duplication identities (B.14). One may indeed check that (3.23) holds thanks to the modular identity

$$
\theta_{E_{8}[1]}^{2}=\theta_{E_{8}[2], 1}^{2}+120 \theta_{E_{8}[2], 248}^{2}+135 \theta_{E_{8}[2], 3875}^{2}
$$

For a fixed electric charge vector $2 \Sigma+\mathcal{P}$, the untwisted DH states (irrespective of their oscillator level) are thus enumerated by

$$
\frac{1}{2} \frac{\Theta_{E_{8}[2], \mathcal{P}}(\tau)}{\eta^{24}}+\frac{1}{2} \delta_{\mathcal{P}, 0} e^{2 \pi i \delta \cdot P_{3}} \frac{2^{4}}{\eta^{12} \vartheta_{2}^{4}}:=q^{\Delta_{\mathcal{P}}} \sum_{N=0}^{\infty} \Omega_{\mathcal{P}}^{u}(N) q^{N}
$$

where $N+\Delta_{\mathcal{P}}=\frac{1}{2} Q^{2}$. Notice that the second term on the left-hand side corresponds to states with charges $P_{1}=P_{2}$, hence $\mathcal{P}=\Delta=0$. 


\section{Twisted sector}

Let us now analyze the DH states in the twisted sectors. Many details are easily obtained by taking the modular transform of the partition function with boundary conditions $(1, g)$. Unlike the untwisted sector, twisted states automatically have $P_{1}=$ $P_{2}$, however their charges now take values in

$$
M_{1}=E_{8}\left(-\frac{1}{2}\right) \oplus\left(I I^{1,1}+\delta\right) \oplus I I^{5,5}
$$

This is not a lattice since a sum of two vectors in $M_{1}$ ends up in $M_{0}$. DH states take the form

$$
\mathcal{P}^{ \pm}(\alpha)\left(1 \mp e^{i \pi\left(\frac{1}{2} P^{2}+\left(P_{3}+\delta\right)^{2}\right)}\right)\left|P ; P_{3}+\delta, P_{4}\right\rangle \otimes|t\rangle \otimes|\tilde{s}\rangle
$$

where $|t\rangle$ is the twisted left-moving ground state, and 8 of the bosonic oscillators in $\mathcal{P}^{ \pm}(\alpha)$ are half-integer modded. DH states with electric charges $Q_{e}=\left(P ; P_{3}+\delta, P_{4}\right) \in$ $M_{1}$ are now enumerated by the partition function

$$
\frac{1}{2}\left(\frac{1}{\eta^{12} \vartheta_{4}^{4}} \pm \frac{1}{\eta^{12} \vartheta_{3}^{4}}\right):=q^{\Delta_{ \pm}} \sum_{N=0}^{\infty} \Omega_{ \pm}^{t}(N) q^{N}
$$

where the sign is that of $-e^{i \pi\left(\frac{1}{2} P^{2}+\left(P_{3}+\delta\right)^{2}\right)}$, and $\Delta_{+}=-\frac{1}{2}, \Delta_{-}=0$. By the level matching condition (3.15), $N+\Delta_{ \pm}$is equated to the square of the electric charge $Q^{2} / 2$.

\section{Comparison with macroscopic prediction}

Having obtained the exact degeneracies in the untwisted and twisted sectors, we may now extract their asymptotics using the Rademacher formula (A.5),

$\Omega_{a b s}(Q)=\frac{1}{2} \hat{I}_{9}\left(4 \pi \sqrt{Q^{2} / 2}\right)+2^{-6} \hat{I}_{9}\left(4 \pi \sqrt{Q^{2} / 4}\right)\left\{\begin{array}{cc}15+16 e^{2 \pi i P_{3} \cdot \delta}, & \mathcal{P} \in \mathcal{O}_{1} \\ 1, & \mathcal{P} \in \mathcal{O}_{248} \\ -1, & \mathcal{P} \in \mathcal{O}_{3875} \\ -e^{i \pi Q^{2}}, & Q \in M_{1}\end{array}\right\}+\ldots$

where $Q \in M_{0}$ in the first three cases. Comparing to the general prediction (2.27) for a $\mathcal{N}=2$ theory with $n_{V}=16$ vectors, we see that the microscopic counting (3.30) matches the macroscopic entropy to all orders in $1 / N$, in all sectors. However, the subleading correction depends on the fine details of the charge vector in the lattice $M_{0} \oplus M_{1}$. 


\section{Helicity supertraces}

Finally, it is useful to check the analysis above against a direct computation of the helicity supertraces. The partition function of the $E_{8} \times E_{8}$ heterotic string on $T^{6}$ with an insertion of $e^{i \pi v J_{3}^{R}} e^{i \pi \bar{v} J_{3}^{L}}$ is given by

$$
Z_{(4,16)}^{H}(v, \bar{v})=\frac{\xi(v) \bar{\xi}(\bar{v})}{4 \tau_{2}|\eta|^{4}} \sum_{h, g} \sum_{\alpha, \beta}(-1)^{\alpha+\beta+\alpha \beta} \frac{\bar{\theta}\left[\begin{array}{l}
\alpha / 2 \\
\beta / 2
\end{array}\right](\bar{v}) \bar{\theta}^{3}\left[\begin{array}{c}
\alpha / 2 \\
\beta / 2
\end{array}\right](0)}{\bar{\eta}^{4}} \frac{Z_{6,6}}{|\eta|^{12}} Z_{c u r}\left[\begin{array}{l}
h / 2 \\
g / 2
\end{array}\right]
$$

where $\alpha, \beta=0,1$ run over the four spin structures and $h, g=0,1$ run over the four (untwisted/twisted, unprojected/unprojected) sectors of the orbifold. In the above expression,

$$
Z_{6,6}\left[\begin{array}{l}
h / 2 \\
g / 2
\end{array}\right]=\sum_{p \in \Gamma_{6,6}}(-1)^{g(\delta, p)} q^{\frac{1}{2} \Pi_{L}^{2}\left(p+\frac{h}{2} \delta\right)} \bar{q}^{\frac{1}{2} \Pi_{R}^{2}\left(p+\frac{h}{2} \delta\right)}
$$

is the partition function for the shifted $\Gamma_{6,6}$ lattice, and

$$
\begin{aligned}
& Z_{\text {cur }}\left[\begin{array}{l}
0 \\
0
\end{array}\right]=\frac{\theta_{E_{8}[1]}^{2}}{\eta^{16}}(\tau), \quad Z_{\text {cur }}\left[\begin{array}{c}
0 \\
\frac{1}{2}
\end{array}\right]=\frac{\theta_{E_{8}[1]}}{\eta^{8}}(2 \tau) \\
& Z_{\text {cur }}\left[\begin{array}{c}
\frac{1}{2} \\
0
\end{array}\right]=\frac{\theta_{E_{8}[1]}}{\eta^{8}}\left(\frac{\tau}{2}\right), \quad Z_{\text {cur }}\left[\begin{array}{c}
\frac{1}{2} \\
\frac{1}{2}
\end{array}\right]=e^{-2 i \pi / 3} \frac{\theta_{E_{8}[1]}}{\eta^{8}}\left(\frac{\tau+1}{2}\right)
\end{aligned}
$$

are the orbifold blocks corresponding to the exchange of the two $E_{8}$ factors. Since the orbifold acts purely on the right-moving part, the helicity partition function is obtained just as in the $(6,22)$ case, leading to the helicity supertraces

$$
\begin{aligned}
B_{4} & =\frac{3}{2 \tau_{2} \eta^{8}} \times \frac{1}{2} \sum_{h, g} Z_{6,6}\left[\begin{array}{l}
h / 2 \\
g / 2
\end{array}\right] Z_{c u r}\left[\begin{array}{l}
h / 2 \\
g / 2
\end{array}\right] \\
B_{6} & =\frac{15\left(2-E_{2}\right)}{8 \tau_{2} \eta^{8}} \times \frac{1}{2} \sum_{h, g} Z_{6,6}\left[\begin{array}{l}
h / 2 \\
g / 2
\end{array}\right] Z_{c u r}\left[\begin{array}{l}
h / 2 \\
g / 2
\end{array}\right]
\end{aligned}
$$

Using the duplication identities (B.14), we obtain

$$
\begin{aligned}
B_{4}= & \frac{3}{2 \tau_{2}} \times \frac{1}{2}\left[\frac{\theta_{E_{8}[1]}^{2}}{\eta^{24}} Z_{6,6}\left[\begin{array}{l}
0 \\
0
\end{array}\right]+2^{4} \frac{\theta_{E_{8}[1]}(2 \tau)}{\theta_{2}^{4} \eta^{12}} Z_{6,6}\left[\begin{array}{l}
0 \\
\frac{1}{2}
\end{array}\right]\right. \\
& \left.+\frac{\theta_{E_{8}[1]}\left(\frac{\tau}{2}\right)}{\theta_{4}^{4} \eta^{12}} Z_{6,6}\left[\begin{array}{c}
\frac{1}{2} \\
0
\end{array}\right]-\frac{\theta_{E_{8}[1]}\left(\frac{\tau+1}{2}\right)}{\theta_{3}^{4} \eta^{12}} Z_{6,6}\left[\begin{array}{c}
\frac{1}{2} \\
\frac{1}{2}
\end{array}\right]\right]
\end{aligned}
$$


where the theta series in the numerator can also be written as

$$
\begin{aligned}
\theta_{E_{8}[1]}(\tau) & =\frac{1}{2}\left(\theta_{3}^{8}+\theta_{4}^{8}+\theta_{2}^{8}\right) \\
\theta_{E_{8}[1]}(2 \tau) & =2^{-4}\left(\theta_{3}^{8}+\theta_{4}^{8}+14 \theta_{3}^{4} \theta_{4}^{4}\right) \\
\theta_{E_{8}[1]}(\tau / 2) & =\theta_{3}^{8}+\theta_{2}^{8}+14 \theta_{3}^{4} \theta_{2}^{4} \\
\theta_{E_{8}[1]}((\tau+1) / 2) & =\theta_{4}^{8}+\theta_{2}^{8}-14 \theta_{4}^{4} \theta_{2}^{4}
\end{aligned}
$$

Using (3.25) above, the untwisted contribution $(h=0)$ may be rewritten as

$$
\begin{aligned}
& \frac{3}{2 \tau_{2}} \times \sum_{\epsilon= \pm 1} \frac{Z_{6,6}\left[\begin{array}{l}
0 \\
0
\end{array}\right]+\epsilon Z_{6,6}\left[\begin{array}{l}
0 \\
\frac{1}{2}
\end{array}\right]}{2}\left[\theta_{E_{8}[2], 1} \times \frac{1}{2}\left(\frac{\theta_{E_{8}[2], 1}}{\eta^{24}}+\epsilon \frac{2^{4} \vartheta_{2}^{4}}{\eta^{12}}\right)\right. \\
& \left.+120 \theta_{E_{8}[2], 248} \times\left(\frac{\theta_{E_{8}[2], 248}}{2 \eta^{24}}\right)+135 \theta_{E_{8}[2], 3875} \times\left(\frac{\theta_{E_{8}[2], 3875}}{2 \eta^{24}}\right)\right]
\end{aligned}
$$

Each term in round brackets can now be interpreted as the multiplicity for the DH states in the conjugacy class $\mathcal{O}_{\mathcal{P}}$ of $M_{0}$ indicated by the $E_{8}$ character which multiplies it. Similarly, in the twisted sector we have

$$
\frac{3}{2 \tau_{2}} \times \sum_{\epsilon= \pm 1} \frac{Z_{6,6}\left[\begin{array}{c}
\frac{1}{2} \\
0
\end{array}\right]+\epsilon Z_{6,6}\left[\begin{array}{c}
\frac{1}{2} \\
\frac{1}{2}
\end{array}\right]}{2} \frac{1}{2}\left[\frac{1}{\theta_{4}^{4} \eta^{12}} \theta_{E_{8}[1]}\left(\frac{\tau}{2}\right)-\epsilon \frac{1}{\theta_{3}^{4} \eta^{12}} \theta_{E_{8}[1]}\left(\frac{\tau+1}{2}\right)\right]
$$

This indeed reproduces the result (3.29) above. It is also clear the generating function of the 6 -th helicity supertrace $B_{6}$ is given by the same partition functions as before, up to a factor $5\left(2-E_{2}\right) / 4$.

\subsection{General Reduced Rank Models}

The agreement found for the $(4,16)$ model of the previous section and the $(4,24)$ model

of Section 2 can in fact be easily seen to generalize to all freely acting $\mathcal{N}=4$ orbifolds of the heterotic string compactified on $T^{6}$ by the following reasoning. In these models, DH states can always be constructed in the untwisted sector, by taking an arbitrary excitation of the left-moving 24 bosons, with appropriate momenta and winding, and ensuring invariance under the discrete symmetry. If $k=24-n_{V}$ is the number of vector fields which are projected out by the orbifold, the generating function for the absolute degeneracies (or, equivalently the helicity supertraces $\Omega_{4}$ ) of DH states in the untwisted sector will take the form

$$
\frac{1}{|G|}\left(\frac{Z}{\eta^{24}}+\sum_{g \in G \backslash\{1\}} \Theta_{g}\right)
$$


where $|G|$ is the order of the orbifold group, $Z$ is the partition of the lattice of charges which have been projected out, and $\Theta_{g}$ are the partition function with an insertion of the generator $g \in G$. Indeed, the $k$ charges are not physical and correspond to internal degrees of freedom. The first term in (3.45) is a modular form of weight $k / 2-12=-n_{V} / 2$ and, provided the left-moving ground state is invariant under the orbifold, has leading term $1 / q$. The Rademacher formula gives a Bessel function of the required order $1-w=\left(n_{V}+2\right) / 2$,

$$
\Omega^{u}(Q) \sim \hat{I}_{\left(n_{V}+2\right) / 2}\left(4 \pi \sqrt{Q^{2} / 2}\right)
$$

in agreement with the prediction (2.27). The other terms have the same modular weight, but mix with twisted sectors under modular transformation, and as a result are exponentially suppressed. In the twisted sectors, the generating functions can be obtained by modular invariance, hence have the same modular weight. Their mixing with the untwisted terms $\Theta_{g}$ implies that the leading term in the Rademacher expansion is controlled by the same pole with $\Delta=1$. Thus, the agreement with the OSV prediction (2.27) is expected to hold for all $\mathcal{N}=4$ reduced rank models. This is confirmed by the analysis of other Het $\left(4, n_{V}\right)$ models in Appendix D. As we shall see in Section 5 , the situation is quite different for $\mathcal{N}=2$ models, where the leading term in (3.45) is absent in the case of the helicity supertrace $\Omega_{2}$, or moduli dependent for absolute degeneracies $\Omega_{a b s}$.

\subsection{A Type II $(2,2) /(0,4)$ Dual Pair}

Let us now turn to a different type II model, where the degeneracies of DH states can be computed exactly by using a type II dual, albeit with unusual $(0,4)$ worldsheet supersymmetry [66].

Consider type IIA compactified on the orbifold $\left(T^{4} \times T^{2}\right) / \mathbb{Z}_{2}$, where the orbifold acts by a reversal of the coordinates on $T^{4}$, times a translation along one circle in $T^{2}$. Since the 16 twisted sectors obtain a mass due to the shift, the massless spectrum consists of 6 vector multiplets of $\mathcal{N}=4$, together with the gravity multiplet. The moduli space is thus given by (2.1) with $n_{V}=6$, where the $S l(2)$ factor corresponds to the Kähler modulus of $T^{2}$. This orbifold can be viewed as a variant of a $K 3$ compactification. We shall denote this model by $(2,2)$, reflecting the fact that the $\mathcal{N}=4$ supersymmetries in target space arise from the world-sheet supersymmetry symmetrically between the left and right-movers.

This model was argued to be U-dual to a $(4,0)$ type IIA model, constructed as

the different orbifold $\left(T^{4} \times T^{2}\right) / \mathbb{Z}_{2}$ by $(-1)^{F_{L}}$ (where $F_{L}$ is the left-moving world-sheet fermion number) times a translation along on circle in $T^{2}$ [66]. The orbifold gives a 
mass to all Ramond-Ramond fields, leaving again $6+6$ vector multiplets of $\mathcal{N}=4$. While it has the same $\mathcal{N}=4$ supersymmetry, the latter now comes entirely from the right-moving supercharges on the world-sheet. Just as in the heterotic string, the $S l(2)$ factor in (2.1) now parameterizes the axio-dilaton. The duality between these $(0,4)$ and $(2,2)$ models is thus very similar to the usual heterotic $/ T^{6}$ - type IIA $/ K 3 \times T^{2}$ duality.

Just as in the heterotic $/ T^{6}$ case, DH states of the $(0,4)$ model can be constructed by exciting the left-movers only, combined with appropriate momenta and winding along $T^{6}$. Their helicity supertraces have been computed in [61] (Eq. 6.11):

$$
\begin{aligned}
B_{4} & =\frac{3}{2} \sum_{(h, g) \neq(0,0)} H_{4}\left[\begin{array}{l}
h / 2 \\
g / 2
\end{array}\right] Z_{2,2}\left[\begin{array}{l}
h / 2 \\
g / 2
\end{array}\right], \\
B_{6} & =\frac{15}{8} \sum_{(h, g) \neq(0,0)}\left(H_{4}\left[\begin{array}{l}
h / 2 \\
g / 2
\end{array}\right]+H_{6}\left[\begin{array}{l}
h / 2 \\
g / 2
\end{array}\right]\right) Z_{2,2}\left[\begin{array}{l}
h / 2 \\
g / 2
\end{array}\right] Z_{4,4}
\end{aligned}
$$

where

$$
H_{4}\left[\begin{array}{l}
h / 2 \\
g / 2
\end{array}\right]=e^{i \pi g} \frac{\theta^{4}\left[\begin{array}{l}
h \\
g
\end{array}\right]}{\eta^{12}}, \quad H_{6}\left[\begin{array}{l}
h / 2 \\
g / 2
\end{array}\right]= \begin{cases}\frac{\theta_{3}^{8}-\theta_{4}^{8}}{2 \eta^{12}}, & (h, g)=(0,1) \\
\frac{\theta_{2}^{8}-\theta_{3}^{8}}{2 \eta^{12}}, & (h, g)=(1,0) \\
\frac{\theta_{4}^{8}-\theta_{2}^{8}}{2 \eta^{12}}, & (h, g)=(1,1)\end{cases}
$$

Note that the contribution of the $(h, g)=(0,0)$ sector vanishes as it has $N=8$ supersymmetry. From these expressions it easy to disentangle the contributions of the various sectors: the degeneracies of DH states in the untwisted sector are generated by

$$
\frac{1}{2} \frac{\theta_{3}^{4}-\theta_{4}^{4}}{\eta^{12}} \text { or } \frac{1}{2} \frac{\theta_{2}^{4}}{\eta^{12}}
$$

depending whether the momentum along the shifted circle in $T^{2}$ is even or odd, respectively. Those in the twisted sector are given by the same expressions for odd and even momentum, respectively. In either case, the degeneracies grow as

$$
\Omega_{4}=\frac{3}{2} \Omega_{a b s} \sim 3 \cdot 2^{5} \hat{I}_{5}\left(2 \pi \sqrt{Q^{2} / 2}\right),
$$

hence have half the entropy of the DH states in the heterotic $(4,24)$ model. As in that model, the helicity supertrace $\Omega_{6}$ originates entirely from $1 / 2$-BPS DH states, and the perturbative string spectrum contains no intermediate multiplets.

Let us now turn to the type II $(2,2)$ side, and see if this entropy may be accounted for by higher derivative interactions. The $R^{2}$ amplitude in the $(2,2)$ model has been obtained by a one-loop computation in [61] (Sec. 6.1):

$$
f_{R^{2}}=8 \log T_{2}\left|\theta_{4}(T)\right|^{4}
$$


where we use the same normalization as in (3.9). In contrast to the other $\mathcal{N}=4$ type II model considered in this section, this amplitude contains only worldsheet instantons (except for the logarithmic term), and vanishes in the large volume limit $T \rightarrow i \infty$. This is in agreement with the fact that, on the $(0,4)$ side, the tree-level higher-derivative corrections start at order $R^{4}$ corrections, as expected for an orbifold of type IIA. In particular, the geometry remains singular, and the OSV formula appears to be unable to reproduce the microscopic entropy in (3.51). It would be interesting to see if $R^{4}$ corrections can resolve the singularity.

Finally, let us note that the $(2,2)$ model also has purely electric DH states, analogous to the states discussed in Section 2.7. Their helicity supertraces have been computed in [61] (Eq. 6.4). In contrast to the type $I I A / K^{3} \times T^{2}$ case, the $1 / 4$-BPS states do not entirely cancel from the helicity supertraces, instead the latter are given by modular forms of positive weight,

$$
\begin{aligned}
B_{4} & =12 \sum_{(h, g) \neq(0,0)} Z_{2,2}\left[\begin{array}{l}
h / 2 \\
g / 2
\end{array}\right] \\
B_{6} & =\frac{15}{2} \sum_{(h, g) \neq(0,0)}\left(4+H_{2}\left[\begin{array}{l}
h / 2 \\
g / 2
\end{array}\right]+\bar{H}_{2}\left[\begin{array}{l}
h / 2 \\
g / 2
\end{array}\right]\right) Z_{2,2}\left[\begin{array}{l}
h / 2 \\
g / 2
\end{array}\right]
\end{aligned}
$$

where

$$
H_{2}\left[\begin{array}{c}
h / 2 \\
g / 2
\end{array}\right]= \begin{cases}\theta_{3}^{4}+\theta_{4}^{4}, & (h, g)=(0,1) \\
-\theta_{2}^{4}-\theta_{3}^{4}, & (h, g)=(1,0) \\
\theta_{2}^{4}-\theta_{4}^{4}, & (h, g)=(1,1)\end{cases}
$$

Depending on the sign of $Q^{2}$, the helicity supertrace $\Omega_{6}$ of $1 / 4$-BPS states is generated by either $H_{2}$ or $\bar{H}_{2}$ in (3.53). Since the modular weight of the counting function is positive, the helicity supertrace $\Omega_{6}$ grows as a power of the charges, rather than exponentially. In contrast, absolute degeneracies are counted by the same functions as in (3.50), hence have an entropy of order $2 \pi \sqrt{Q^{2} / 2}$. Just as in the type $\mathrm{II} / K 3 \times T^{2}$ case, it would be interesting to understand how these states acquire a smooth horizon.

\section{Macroscopic Predictions for Extremal Black Holes Degen- eracies in $\mathcal{N}=2$ Models}

In this section, we return to the realm of $\mathcal{N}=2$ supersymmetry, where the OSV conjecture was originally formulated, and extract the degeneracies of extremal black holes as predicted by the conjectural relation (1.7). We start in Subsection 4.1 by 
reviewing the relation between the generalized prepotential and the topological string amplitude. We then evaluate (1.7) for large black holes with no D6-brane charge $\left(p^{0}=0\right)$, in particular scaling limits of the charges. The case of small black holes in $K 3$-fibrations is discussed in Subsection 4.3. Finally, in Subsection 4.4 we compute the integral (1.7) for arbitrary D6-brane charge, for tree-level prepotentials of the form $F=X^{1} X^{a} C_{a b} X^{b} / X^{0}$. This is a special example of the Legendre invariant prepotentials discussed in [67].

\subsection{Generalized Prepotential and the Topological String Amplitude}

As we recalled in the introduction, $\mathcal{N}=2$ supergravity admits an infinite series of higher-derivative corrections which can be written as integral of a chiral density in $\mathcal{N}=2$ superspace,

$$
\begin{aligned}
\int d^{4} \theta F\left(X^{I}, W^{2}\right) & =\int d^{4} \theta \sum_{h=0}^{\infty} F_{h}\left(X^{A}\right) W^{2 h} \\
& =\mathcal{L}_{\text {tree }}+\sum_{h=1}^{\infty} F_{h}\left(X^{A}\right)\left({ }^{-} C^{-}\right)^{2}\left(T^{-}\right)^{2 h-2}+\ldots
\end{aligned}
$$

where $X^{I}\left(I=0 . . n_{V}-1\right)$ are the homogeneous superfields for the vector multiplets, $W$ is the $N=2$ Weyl superfield, with $W^{2}=\left(T^{-}\right)^{2}+\cdots+\theta^{4}\left({ }^{-} C^{-}\right)^{2}$, and the ellipses denote other interactions related by supersymmetry (see e.g. [68] for a review of this formalism). In the above expression, ${ }^{-} C^{-}$denotes the anti-self-dual part of the Weyl tensor, $T^{-}$the anti-self-dual part of the graviphoton field-strength. For $h=0$, one recovers the two-derivative $N=2$ Lagrangian controlled by the prepotential $F_{0}\left(X^{I}\right)$

For $\mathcal{N}=2$ models obtained by compactifying type IIA string theory on a CalabiYau three-fold, it can be shown that the only contribution to the $\left({ }^{-} C^{-}\right)^{2}\left(T^{-}\right)^{2 h-2}$ coupling (or its on-shell equivalent $\left({ }^{-} R^{-}\right)^{2}\left(T^{-}\right)^{2 h-2}$ ) occurs at genus $h$, and reduces to a vacuum amplitude in the A-model topological string, obtained from the $(2,2)$ superconformal sigma model on $\mathcal{X}$ by a topological twist $[20,69]$. In general, it includes non-holormophic contributions from massless states propagating in the loops. The holomorphic topological string amplitude is defined as an asymptotic expansion in the topological string coupling near some large radius limit (i.e. in a neighborhood of a point of maximal unipotent monodromy). It includes perturbative contributions ${ }^{15}$ at genus 0 and 1, together with an infinite sum of world-sheet instanton contributions at arbitrary genera,

$$
F_{\text {top }}=-\frac{i(2 \pi)^{3}}{6 \lambda^{2}} C_{A B C} t^{A} t^{B} t^{C}-\frac{i \pi}{12} c_{2 A} t^{A}+F_{G W}(\lambda, q)
$$

\footnotetext{
${ }^{15}$ In general one should allow for an extra quadratic polynomial in $t^{a}$ with real coefficients. These terms can be reabsorbed by a change of variable and do not play any role in our discussion.
} 
where $\lambda$ is the topological string coupling ${ }^{16}, t^{A}=\theta^{A}+i r^{A}$ with $r^{A}>0$ are the complexified Kähler moduli on a basis $\gamma^{A}$ of $H_{2}(\mathcal{X}, \mathbb{Z})\left(A=1, \ldots, n_{V}-1\right), C_{A B C}$ are the triple intersection numbers $C_{A B C}=\int_{\mathcal{X}} J_{A} J_{B} J_{C}, c_{2 A}=\int_{\mathcal{X}} J_{A} c_{2}\left(T^{1,0} \mathcal{X}\right)$, and

$$
\begin{aligned}
F_{G W}(\lambda, q) & =\sum_{h \geq 0, \beta} N_{h, \beta} q^{\beta} \lambda^{2 h-2} \\
& =\sum_{h \geq 0, \beta, d \geq 1} n_{\beta}^{h} \frac{1}{d}\left(2 \sin \frac{d \lambda}{2}\right)^{2 h-2} q^{d \beta} .
\end{aligned}
$$

is the Gromov-Witten instanton sum. Here $\beta=\beta^{A} \gamma_{A}$ runs over effective curves with $\beta^{A} \in \mathbb{Z}^{+}, q^{\beta}:=e^{2 \pi i \beta_{A} t^{A}}$, and $N_{h, \beta}$ are the (rational) Gromov-Witten invariants. In the second line we have used the identity of Gopakumar and Vafa to rewrite $F_{G W}$ in terms of integral BPS invariants $n_{\beta}^{h}$.

The precise relation between the topological string amplitude and the generalized prepotential is

$$
F_{\text {top }}\left(t^{A}, \lambda\right)=\frac{i \pi}{2} F_{S U G R A}\left(X^{A}, W^{2}\right), \quad t^{A}=\frac{X^{A}}{X^{0}}, \quad \lambda^{2}=\left(\frac{\pi}{4} \frac{W}{X^{0}}\right)^{2}
$$

leading to the standard supergravity normalization ${ }^{17}$

$$
F_{\text {sugra }}=-\frac{1}{6} C_{A B C} \frac{X^{A} X^{B} X^{C}}{X^{0}}-\frac{W^{2}}{64} \frac{c_{2 A}}{24} \frac{X^{A}}{X^{0}}-\frac{X^{0^{2}}}{(2 \pi i)^{3}} \sum_{h, \beta} N_{h, \beta} q^{\beta}\left(\frac{\pi W}{4 X^{0}}\right)^{2 h}
$$

It is important to note that the sum in $(4.4 \mathrm{~b})$ contains degenerate instanton contributions, with $\beta=0$. Those occur only at genus 0 , and are controlled by the single BPS invariant $n_{0}^{0}=-(1 / 2) \chi(\mathcal{X})$, where $\chi$ is the Euler number of $\mathcal{X}$ :

$$
F_{G W}^{\mathrm{deg}}(\lambda)=-\frac{1}{2} \chi(\mathcal{X}) f(\lambda):=-\frac{1}{2} \chi(\mathcal{X}) \sum_{d=1}^{\infty} \frac{1}{d} \frac{1}{\left(2 \sin \frac{d \lambda}{2}\right)^{2}}
$$

where the second equality defines the Mac-Mahon function $f(\lambda) . \quad F_{G W}^{\text {deg }}$ admits an asymptotic expansion at weak topological coupling,

$$
F_{G W}^{d e g}=-\frac{1}{2} \chi(\mathcal{X})\left[\lambda^{-2} \zeta(3)+K-\sum_{n=0}^{\infty} \lambda^{2 n+2} \frac{\left|B_{2 n+4}\right|}{(2 n+4) !} \frac{(2 n+3)}{(2 n+2)} B_{2 n+2}\right]
$$

\footnotetext{
${ }^{16}$ In the notations of $[14], \lambda^{2}=-g_{\text {top }}^{2}$.

${ }^{17}$ The factor of proportionality relating $\lambda$ and $W / X^{0}$ can be obtained by demanding the correct automorphic result for IIA $/ K 3 \times T^{2}$.
} 
where the "constant" $K$ is computed in Appendix E,

$$
K=\frac{1}{12} \log \frac{2 \pi i}{\lambda}-\frac{1}{2 \pi^{2}} \zeta^{\prime}(2)+\frac{1}{12} \gamma_{E}
$$

In equation (4.8) above, the $O\left(1 / \lambda^{2}\right)$ term corresponds to the famous contribution to the prepotential coming from the reduction of the tree-level $R^{4}$ coupling in 10 dimensions [70], and the coefficient of $\lambda^{2 n+2}$ is the Euler character of the moduli space of genus $n+2$, as computed in [71]. The "constant" $K$ depends logarithmically on $\lambda$, hence cannot be attributed to any order in the genus expansion. Nevertheless, it follows from a careful analysis of the weak coupling behavior of $f(\lambda)$, which is analytic for $\operatorname{Im} \lambda \neq 0$. This term is usually dropped in the topological string literature, but will play an important role in the analysis of the black hole degeneracies below.

Instead, for $N=2$ backgrounds obtained by compactifying the heterotic string on $K 3 \times T^{2}$, the higher-derivative coupling $\left({ }^{-} C^{-}\right)^{2}\left(T^{-}\right)^{2 h-2}$ for any $h$ receives contributions at 1-loop already [72] (as well as tree-level for $h=0,1$ ). In fact, using heterotic-type II duality, this is a powerful way to compute the Gromov-Witten invariants of compact K3-fibered Calabi-Yau three-folds, at least for effective curves $\beta$ lying only in the K3 fiber [73] (see [74] for recent progress).

Finally, let us note that by expanding the parenthesis in (4.4b) in binomial series and summing term by term over $d$, we may rewrite

$$
\begin{aligned}
F_{G W}= & \sum_{\beta} \sum_{k=1}^{\infty} k n_{\beta}^{0} \log \left(1-e^{i k \lambda}\right)-\sum_{\beta} n_{\beta}^{1} \log \left(1-q^{\beta}\right) \\
& +\sum_{h \geq 2} \sum_{\beta} \sum_{l=0}^{2 h-2}(-1)^{h+l}\left(\begin{array}{c}
2 h-2 \\
\ell
\end{array}\right) n_{\beta}^{h} \log \left(1-q^{\beta} e^{i(h-1-l) \lambda}\right)
\end{aligned}
$$

hence obtaining $\exp \left(F_{G W}\right)$ as an infinite product $[75,76]$. Unfortunately, for $h \geq 2$ the infinite product is in general divergent, falling short of providing a non-perturbative definition of the topological string amplitude.

\subsection{Large Black Holes with $p^{0}=0$}

Let us now turn to the evaluation of the integral (1.7), for large black holes, with non-zero entropy at the classical level. Since their entropy at large charges is already well reproduced by the tree-level prepotential, it is natural to expect that GromovWitten instantons can be neglected, at least in some large charge regime. Under this assumption (to which we shall return below), and restricting to $p^{0}=0$ for simplicity (see [67] for a discussion of the $p^{0} \neq 0$ case), the free energy (1.2) reads

$$
\mathcal{F}^{\text {pert }}=-\frac{\pi}{6} \frac{\hat{C}(p)}{\phi^{0}}+\frac{\pi}{2} \frac{C_{A B}(p) \phi^{A} \phi^{B}}{\phi^{0}}
$$


where we use the standard notation

$$
C_{A B}(p)=C_{A B C} p^{C}, \quad C(p)=C_{A B C} p^{A} p^{B} p^{C}, \quad \hat{C}(p)=C(p)+c_{2 A} p^{A} .
$$

Note in particular that, in this limit, the only effect of higher derivative corrections is to replace $C(p) \rightarrow \hat{C}(p)$.

We further assume that the measure $[d \phi]$ is the standard Euclidean measure, extending over the infinite real axis or some deformation thereof. The integral over $\phi^{A}$ is therefore Gaussian, with a peak at

$$
\phi_{*}^{A}=-C^{A B}(p) q_{B} \phi^{0}
$$

Due to the indefinite signature of the quadratic form $C_{A B}(p)$, it is well defined only upon rotating the contour of integration so that $\phi^{A} / \sqrt{\phi^{0}} \sim e^{ \pm i \pi / 4}$. Proceeding formally, we find

$$
\Omega\left(p^{A}, q_{A}\right) \sim \int d \phi^{0} \frac{\left(2 \phi^{0}\right)^{\frac{n_{V}-1}{2}}}{\left|\operatorname{det} C_{A B}(p)\right|^{1 / 2}} \exp \left(-\frac{\pi}{6} \frac{\hat{C}(p)}{\phi^{0}}+\pi \phi^{0} \hat{q}^{0}\right)
$$

where $C^{A B}(p)$ is the inverse matrix of $C_{A B}(p)$ and

$$
\hat{q}_{0}=q_{0}-\frac{1}{2} q_{A} C^{A B}(p) q_{B}
$$

is invariant under unipotent monodromies. The integral over $\phi^{0}$ is now of Bessel type, with a saddle point at

$$
\phi_{*}^{0}= \pm \sqrt{\frac{-\hat{C}(p)}{6 \hat{q}_{0}}}
$$

When $\hat{q}_{0}<0$, the action at the saddle point is real, and equal to

$$
S_{0}=2 \pi \sqrt{-\hat{C}(p) \hat{q}_{0} / 6}
$$

Provided the saddle point is actually selected by the contour integral, we thus find that the formula (1.7) predicts

$$
\Omega\left(p^{A}, q_{A}\right) \sim \pm \frac{1}{2}\left|\operatorname{det} C_{a b}(p)\right|^{-1 / 2}(\hat{C}(p) / 6)^{\nu} \times \hat{I}_{\nu}\left(2 \pi \sqrt{-\hat{C}(p) \hat{q}_{0} / 6}\right)
$$

where

$$
\nu=\frac{1}{2}\left(n_{V}+1\right)
$$

Using the asymptotic expansion (2.13), we thus find

$$
\log \Omega\left(p^{A}, q_{A}\right) \sim S_{0}-\frac{1}{2}\left(n_{V}+1\right) \log \left(S_{0} / 4 \pi\right)-\log \mathcal{N}(p)+\ldots
$$


where $\mathcal{N}(p)$ is the $p$-dependent prefactor in (4.18), and the ellipses denote an infinite number of calculable power-suppressed contributions. The first term in this equation reproduces the classic result of [44] (generalized to $q^{A} \neq 0$ ), which was successfully matched to the microscopic counting based on M5-branes wrapping a 4-cycle in $\mathcal{X}$.

Let us now discuss the validity of our assumptions. Since this has already been discussed in [1], we shall be brief:

- Upon scaling all electric and magnetic charges to infinity (but keeping $p^{0}=0$ ), the topological coupling $\lambda=4 \pi /\left(i \phi_{*}^{0}\right)$ at the saddle point goes to zero, hence all higher derivative corrections can be neglected. However, the Kähler classes at the saddle point $\operatorname{Im} t^{A}=p^{A} / \phi_{*}^{0}$ stay of order 1 , so it is not legitimate to drop the Gromov-Witten instantons.

- If all $p^{A} \neq 0$ (but $p^{0}=0$ ), it is possible to stay at weak topological coupling and get rid of the Gromov-Witten instantons by scaling $\hat{q}^{0}$ faster than $p^{A}$. In this case, the leading correction to the entropy comes from the tree-level $\zeta(3)$ term in (4.8), which perturbs the saddle point. This predicts a correction ${ }^{18}$ to the Bekenstein-Hawking entropy

$$
S\left(p^{A}, q_{A}\right)=2 \pi \sqrt{-\hat{C}(p) \hat{q}_{0} / 6}+\frac{\zeta(3) \chi(\mathcal{X})}{96 \pi^{2}} \frac{\hat{C}(p)}{\hat{q}_{0}}+\ldots
$$

which still grows like a power of the charges.

- On the microscopic side, the leading entropy is well reproduced from the M5brane conformal field theory when the Ramanujan-Hardy formula is applicable, i.e. when $\hat{q}^{0} \gg \hat{C}(p)$. In this regime, the topological coupling at the saddle point is strong, although the Kähler classes can still be taken to be large. This means that non-degenerate Gromov-Witten instantons could be neglected, provided the BPS invariants grow sufficiently slowly. However, the series of degenerate instantons is strongly coupled, and one should instead use the Gopakumar-Vafa representation in terms of the Mac-Mahon function, which is exponentially suppressed at large coupling. The $\log \lambda$ term in (4.9) implies an extra factor $\left(\phi^{0}\right)^{\chi(\mathcal{X}) / 24}$ in (4.14), which would affect the index of the Bessel function in (4.19). Since (4.14) will be further supported by the microscopic analysis, we propose to modify by hand the definition of the topological string amplitude $\Psi_{\text {top }}$ into

$$
\tilde{\Psi}_{\text {top }}:=\lambda^{\chi / 24} \Phi_{\text {top }}
$$

\footnotetext{
${ }^{18}$ A similar correction was computed in [77], without taking into account the contrubution from the measure.
} 
More generally, it would be interesting to have a better understanding of the integration measure in (1.7).

To summarize, provided the OSV conjecture (1.7) holds, the infinite number of power-suppressed corrections encapsulated in the Bessel function (4.18) can be trusted in the strong coupling regime $\hat{q}^{0} \gg \hat{C}(p)$, provided the Gopakumar-Vafa infinite product is convergent.

Regrettably $^{19}$, there are no examples where the degeneracies of large black holes are known exactly. In principle the index $\Omega_{2}$ should be computable from a $(0,4)$ sigma model described in $[44,78]$, presumably from the elliptic genus of this model. While the sigma model is rather complicated, and has not been well investigated we should note that from the Rademacher expansion it is clear that the leading exponential asymptotics of negative weight modular forms depends on very little data. Essentially all that enters is the order of the pole and the negative modular weight. There are $c_{L}=C(p)+c_{2} \cdot p=\hat{C}(p)$ real left-moving bosons. Since the sigma model is unitary, the relevant modular form has the expansion $q^{-c_{L} / 24}+\cdots$. This gives the order of the pole, and thus we need only know the modular weight. This in turn depends on the number of left-moving noncompact bosons. Each noncompact boson contributes $w=-\frac{1}{2}$ to the modular weight. Now, the sigma model of [44] splits into a product of a relatively simple "universal factor" and a rather complicated "entropic factor," as described in [78]. Little is known about the entropic factor other than that it is a $(0,4)$ conformal theory with $c_{R}=6 k$, where $k=\frac{1}{6} C(p)+\frac{1}{12} c_{2} \cdot p-1$, where $p \in H^{2}(\mathcal{X}, \mathbb{Z})$. The local geometry of the target space was worked out in [78]. Based on this picture we will assume the target space is compact and does not contribute to the modular weight. (Quite possibly the model is a "singular conformal field theory" in the sense of [79] because the surface in the linear system $|p|$ can degenerate along the discriminant locus. It is reasonable to model this degeneration using a Liouville theory, as in [79]. If this is the case we expect the entropic factor to contribute order one modular weight.) The universal factor is much more explicit. The target is $\mathbb{R}^{3} \times S^{1}$, it has $(0,4)$ supersymmetry with $k=1$ and there are $h-1$ (where $h=h_{1,1}$ ) compact leftmoving bosons which are $N=4$ singlets. They have momentum in the anti-selfdual part of $H^{1,1}(\mathcal{X}, \mathbb{Z})$ (anti-self-duality is defined by the surface in $|p|$ ). Since we fix these momenta we obtain $w=-\frac{1}{2}(h-1)$. Finally there are 3 noncompact left-moving bosons describing the center of mass of the black hole in $\mathbb{R}^{3}$. Thus, the net left-moving modular weight is $-(h+2) / 2$. Now, applying the Rademacher expansion in the region

\footnotetext{
${ }^{19}$ The remainder of this section is excerpted from [1].
} 
$\left|\hat{q}_{0}\right| \gg \hat{C}(p)$ we find the elliptic genus is proportional to

$$
\hat{I}_{\nu}\left(2 \pi \sqrt{\frac{\left|\hat{q}_{0}\right| \hat{C}(p)}{6}}\right)
$$

with $\nu=\frac{h+4}{2}$. This is remarkably close to (4.18) ! Clearly, further work is needed here since it is likely there are a number of important subtleties in the entropic factor. Nevertheless, our argument suggests that a deeper investigation of the elliptic genus in this model will lead to an interesting test of (1.7) (or rather (4.22), since it must be done at strong topological string coupling) for the case of large black holes.

\subsection{Small Black Holes}

We now turn to the case of small black holes with $C(p)=0$ but $\hat{C}(p) \neq 0$ : these are singular solutions of the tree-level $\mathcal{N}=2$ supergravity Lagrangian, but it is expected that quantum corrections will smooth out the singularity and lead to a bona fide black hole. For such charges, the matrix $C_{A B}(p)$ is not invertible and some of the manipulations in the previous section need to be rethought.

We are particularly interested in the case when $\mathcal{X}$ is a $K 3$ fibration over $\mathbf{P}^{1}$ admitting a heterotic dual. In this case, we can divide up the special coordinates so that $X^{1} / X^{0}$ is the volume of the base and $X^{a} / X^{0}, a=2, \ldots n_{V}-1$ are associated with the (invariant part of the) Picard lattice of the fiber. The cubic intersection form becomes

$$
-\frac{1}{6} C_{A B C} X^{A} X^{B} X^{C}=-\frac{1}{2} C_{a b} X^{1} X^{a} X^{b}-\frac{1}{6} C_{a b c} X^{a} X^{b} X^{c}
$$

where the indices $a, b$ run from 2 to $n_{V}-1$, and $C_{a b}$ is the intersection form of the (invariant part of the) Picard lattice of the fiber ${ }^{20}$ The matrix $C_{A B}(p)$ thus takes the form

$$
C_{A B}(p)=\left(\begin{array}{cc}
0 & C_{a b} p^{b} \\
C_{a b} p^{b} & p^{1} \tilde{C}_{a b}+C_{a b c} p^{c}
\end{array}\right)
$$

We now specialize to heterotic DH states, with charges $p^{0}=0, p^{a}=0, a=2, \ldots, n_{V}-1$, and $q_{1}=0$, with $p^{1} q_{0} \neq 0$ and $q_{a} \neq 0$ for $a=2, \ldots, n_{V}-1$. Using $c_{1}=24$ for the K3 fiber, the integral (1.7) now becomes

$$
\Omega\left(p^{1}, q_{0}, q_{a}\right)=\int d \phi^{0} d \phi^{1} d \phi^{a} \exp \left(-4 \pi \frac{p^{1}}{\phi^{0}}+\frac{\pi}{2} \frac{p^{1} C_{a b} \phi^{a} \phi^{b}}{\phi^{0}}+\pi q_{0} \phi^{0}\right)
$$

The $\phi^{1}$ dependence disappears from the integrand and one must make a discrete identification on $\theta=\phi^{1} / \phi^{0}$. As in the benchmark case in Section 2, we find that (1.7)

\footnotetext{
${ }^{20}$ Notice that $C_{a b c}=0$ at tree-level on the heterotic side, but not on the type II side in general.
} 
gives

$$
\left(p^{1}\right)^{2} \hat{I}_{\nu}\left(4 \pi \sqrt{\left|p^{1} q_{0}-\frac{1}{2} q_{a} \tilde{C}^{a b} q_{b}\right|}\right)
$$

where the index of the Bessel function is now

$$
\nu=\frac{1}{2}\left(n_{V}+2\right)
$$

Let us now re-discuss the validity of our assumption that Gromov-Witten instantons could be neglected in the small black hole case. Since $C(p)=0$ and $\operatorname{Im} t^{a}=-p^{a} / \phi_{*}^{0}$ at the saddle point, the attractor values of the Kähler moduli are necessarily at the boundary of the Kähler cone. In principle, one must retain the full worldsheet instanton series (or rather, its analytic continuation, should it exist.)

Remarkably $^{21}$, for $\mathcal{N}=4$ compactifications this is not a problem. In this case, due to the decoupling between the two factors in (2.1), $F_{\text {top }}$ is only a function of a single Kähler modulus $t^{1}$, and moreover $\chi(\mathcal{X})=0$. Hence, at the saddle-point,

$$
\phi_{*}^{0}=-\sqrt{\frac{4 p^{1}}{\left|\hat{q}_{0}\right|}} \quad \operatorname{Im} t^{1}=\frac{1}{2} \sqrt{p^{1}\left|\hat{q}_{0}\right|}
$$

Thus, whether or not the topological string coupling is strong $\left(\left|\hat{q}_{0}\right| \gg p^{1}\right)$ or weak $\left(p^{1} \gg\left|\hat{q}_{0}\right|\right)$ the relevant Kähler class is large and the Bessel asymptotics (4.27) are justified.

The situation is rather different for $\mathcal{N}=2$ compactifications. In this case $F_{\text {top }}$ is in general a function of $t^{1}$ as well as $t^{a}$ for $a \geq 2$. Thus the computation in (4.26) is not justified. We stress that the problem is not that the topological string is strongly coupled. Indeed, for $\chi=0$ examples such as the FHSV example discussed in Section 5.3 below, the saddlepoint value (4.29) can be taken in the weak coupling regime by taking $p^{1} \gg\left|\hat{q}_{0}\right|$. In fact, the difficulty appears to be with the formulation of the integral (1.7) itself for the case of charges of small black holes. Recall that we must evaluate

$$
\mathcal{F}_{\text {top }}:=-\pi \operatorname{Im} F_{\text {top }}\left(p^{I}+i \phi^{I}, 256\right)
$$

Since $X^{a} / X^{0}=\phi^{a} / \phi^{0}$ is real, for $a>1$, one must evaluate the worldsheet instanton sum for real values $t^{a}=\phi^{a} / \phi^{0}$. For some Calabi-Yau manifolds it is possible to analytically continue $F_{0}$ from large radius to small values of $\operatorname{Im} t^{a}$. However we may use the explicit results of $[80,81]$, which express $F_{1} \sim \log \Phi$, where $\Phi$ is an automorphic form for $S O(2, n ; \mathbb{Z})$. It appears that $\operatorname{Im} t^{a}=0$ constitutes a natural boundary of the automorphic form $\Phi$. Thus, in the case of $K 3$ fibrations with heterotic duals the formalism of [14] becomes singular for these charges, even at weak topological string coupling.

\footnotetext{
${ }^{21}$ This paragraph is again excerpted from [1].
} 
Remarkably, if we ignore these subtleties, the formula (4.27) turns out to match perfectly with the asymptotic expansions of twisted sector DH states, as we show below. For untwisted sector DH states the asymptotics do not match with either $\Omega_{a b s}$ nor with $\Omega_{2}$.

\subsection{Large Black Holes with $p^{0} \neq 0$}

Finally, let us evaluate the integral (1.7) for large black holes with non-zero D6-brane charge. For simplicity, we restrict ourselves to $K 3$ fibrations with $C_{a b c}=0$. and $c_{2 A}=0^{22}$, and, as in previous cases, disregard the Gromov-Witten instanton series. For convenience, we drop inessential numerical factors. The computation in this section is a special case of the analysis in [67], which applies for cubic prepotentials $F=I_{3}(X) / X^{0}$ which are invariant under Legendre transform in all variables. When this is not the case, such as in the $S T U+U^{3}$ model, the attractor mechanism is significantly more involved.

From (1.2), one computes the black hole free energy in the mixed ensemble,

$$
\mathcal{F}=\frac{p^{0} \phi^{1}-p^{1} \phi^{0}}{\left(p^{0}\right)^{2}+\left(\phi^{0}\right)^{2}}\left(\frac{1}{2} \vec{\phi}^{2}-\frac{1}{2} \vec{p}^{2}\right)-\frac{p^{0} p^{1}+\phi^{0} \phi^{1}}{\left(p^{0}\right)^{2}+\left(\phi^{0}\right)^{2}}(\vec{p} \vec{\phi})
$$

where $\vec{\phi}^{2}=\phi^{a} \phi^{b} C_{a b}, \vec{p}^{2}=p^{a} p^{b} C_{a b}, \vec{p} \phi \vec{\phi}=p^{a} C_{a b} \phi^{b}$, and determines the microcanonical degeneracies via (1.7). The integral over the potentials $\phi^{a}$ is still Gaussian, leading to

$$
\begin{aligned}
\Omega_{O S V}(p, q) & =\int d \phi^{0} d \phi^{1}\left(\frac{\left(p^{0}\right)^{2}+\left(\phi^{0}\right)^{2}}{p^{0} \phi^{1}-p^{1} \phi^{0}}\right)^{\frac{n_{V}-2}{2}} \\
& \exp \left[\frac{\left[\left(p^{1}\right)^{2}+\left(\phi^{1}\right)^{2}\right] \vec{p}^{2}+\left[\left(p^{0}\right)^{2}+\left(\phi^{0}\right)^{2}\right] \vec{q}^{2}-2\left(p^{0} p^{1}+\phi^{0} \phi^{1}\right) \vec{p} \vec{q}}{2\left(p_{1} \phi^{0}-p^{0} \phi^{1}\right)}+q_{0} \phi^{0}+q_{1} \phi^{1}\right]
\end{aligned}
$$

where $\vec{q}^{2}=q_{a} C^{a b} q_{b}$ and $\vec{p} \vec{q}=p^{a} q_{a}$. In order to compute the integral over $\phi^{0}, \phi^{1}$, let us change variables to

$$
\begin{aligned}
p^{0} \cosh x & =\sqrt{\left(p^{0}\right)^{2}+\left(\phi^{0}\right)^{2}} \\
\left(p^{0}\right)^{2} y & =\left(p^{1} \phi^{0}-p^{0} \phi^{1}\right)\left(\vec{p}^{2}-p^{0} q_{1}\right)
\end{aligned}
$$

with Jacobian $d \phi^{0} d \phi^{1} /(d x d y)=\left(p^{0}\right)^{2} \cosh x /\left(\vec{p}^{2}-2 p^{0} q_{1}\right) / 2$. The argument of the exponential in (4.32) becomes

$$
y+\frac{B^{2} \cosh ^{2} x}{4\left(p^{0}\right)^{2} y}+\frac{A}{p^{0}} \sinh x
$$

\footnotetext{
${ }^{22}$ The case $c_{2,1} \neq 0, c_{2, a}=0$ can be obtained by shifting $\vec{p}^{2} \rightarrow \vec{p}^{2}+\frac{1}{3} c_{2,1}$ in the equations below.
} 
where

$$
\begin{aligned}
& A=-p^{1} \vec{p}^{2}+p^{0}\left(p^{0} q_{0}+p^{1} q_{1}+\vec{p} \vec{q}\right) \\
& B=\sqrt{\left(\vec{p}^{2}-2 p^{0} q_{1}\right)\left[\left(p^{1}\right)^{2} \vec{p}^{2}+\left(p^{0}\right)^{2} \vec{q}^{2}-2 p^{0} p^{1} \vec{p} \vec{q}\right]}
\end{aligned}
$$

Together with the above det, this gives

$$
\left(p^{0}\right)^{2}\left(\vec{p}^{2}-2 p^{0} q_{1}\right)^{\frac{n_{V}-4}{2}} \int(\cosh x)^{h} y^{\frac{2-n_{V}}{2}} \exp \left(y+\frac{B^{2} \cosh ^{2} x}{4\left(p^{0}\right)^{2} y}+\frac{A}{p^{0}} \sinh x\right) d x d y
$$

The integral over $y$ is of Bessel type, leading to

$$
\left(p^{0}\right)^{\frac{n_{V}}{2}}\left(\frac{\vec{p}^{2}-2 p^{0} q_{1}}{B}\right)^{\frac{n_{V}-4}{2}} \int(\cosh x)^{n_{V}-1} \exp \left[\frac{A}{p^{0}} \sinh x\right] I_{\frac{n_{V}-4}{2}}\left(\frac{B}{p^{0}} \cosh x\right) d x
$$

In the limit where all charges are scaled to infinity at the same rate, the integral (4.38) may be evaluated by saddle point approximation: the saddle lies at

$$
\phi^{0}=\frac{A}{S_{0}}, \quad \phi^{1}=\frac{1}{p^{0} S_{0}}\left(A p^{1}+\frac{B^{2}}{\vec{p}^{2}-2 p^{0} q_{1}}\right)
$$

where

$$
S_{0}=\frac{1}{p^{0}} \sqrt{B^{2}-A^{2}}
$$

In particular, the Kähler moduli at the saddle point are given by

$$
\begin{aligned}
\operatorname{Im} t^{1} & ==\frac{p^{0} \phi^{1}-p^{1} \phi^{0}}{\left(p^{0}\right)^{2}+\left(\phi^{0}\right)^{2}}=\frac{2 S_{0}}{\vec{p}^{2}-2 p^{0} q_{1}} \\
\operatorname{Im} t^{a} & =\frac{p^{0} \phi^{a}-p^{a} \phi^{0}}{\left(p^{0}\right)^{2}+\left(\phi^{0}\right)^{2}}=\frac{S_{0}}{B^{2}}\left(\vec{p}^{2}-2 p^{0} q_{1}\right)\left(p^{0} C^{a b} q_{b}-p^{1} p^{a}\right)
\end{aligned}
$$

Including the fluctuation determinant, we obtain

$$
\Omega_{O S V}(p, q) \sim B^{2}\left(\vec{p}^{2}-2 p^{0} q_{1}\right)^{\left(n_{V}-4\right) / 2} S_{0}^{-\left(n_{V}+2\right) / 2} \exp \left(S_{0}\right)
$$

The leading entropy $S_{0}$ in (4.41) agrees with the general result in [82]. Using (4.36), it may be rewritten as

$$
S=\sqrt{\left(p^{0}\right)^{2} q_{0}^{2}+2 p^{0} q_{1} \vec{q}^{2}+2 p^{0} q_{0}\left(p^{1} q_{1}+\vec{p} \vec{q}\right)+\left(p^{1} q_{1}-\vec{p} \vec{q}\right)^{2}-2 p^{1} q_{0} \vec{p}^{2}-\vec{p}^{2} \vec{q}^{2}}
$$

where $\vec{p}^{2}=p^{a} C_{a b} p^{b}, \vec{q}^{2}=q_{a} C^{a b} q_{b}$ and $\vec{p} \vec{q}=p^{a} q_{a}$. Defining $Q=\left(q_{0}, p^{1}, q_{a}\right)$ and $P=$ $\left(p^{0},-q_{1}, p^{a}\right)$, this is recognized as the familiar discriminant

$$
S=\sqrt{(P \cdot P)(Q \cdot Q)-(P \cdot Q)^{2}}
$$


One may check that the result (4.44) agrees with (4.20) in the limit $p^{0} \rightarrow 0$, using the fact that $\operatorname{det}\left(C_{A B}(p)\right)=\left(p^{1}\right)^{h-2} \vec{p}^{2}, C(p)=3 p^{1} \vec{p}^{2}$.

On the other hand, it is important to note that the prefactors in (4.44), which follow from using a trivial integration measure for the electric potentials $\phi^{I}$ in (1.7), are not consistent with T-duality. This problem may be cured by using an appropriate integration measure such as

$$
\tilde{\Omega}_{O S V}(p, q)=\int \frac{d \phi^{0} d \phi^{1} d \phi^{a}}{\left|X^{0}\right|^{n_{V}+2}\left(\operatorname{Im} t^{1}\right)^{2}\left(\operatorname{Im} t^{a} C_{a b} \operatorname{Im} t^{b}\right)^{n_{V} / 2}} e^{\mathcal{F}+\pi q^{A} \phi_{A}}
$$

where, as usual, $X^{I}=p^{I}+i \phi^{I}$ and $t^{A}=X^{A} / X^{0}$. To 1-loop order, this does not change the location of the saddle point (4.42), but simply removes the offending factors in (4.44), leading to

$$
\tilde{\Omega}_{O S V}(p, q) \sim S_{0}^{-\left(n_{V}+2\right) / 2} \exp \left(S_{0}\right)
$$

For $p^{0}=0$, the measure in (4.47) reduces to the flat integration measure used in (4.14), up to an overall factor $[C(p)]^{2}$ which depends on the magnetic charges only. However, there is no guarantee that this prescription will be consistent with T-duality at higher orders.

The measure (4.48) is obviously not the only choice which removes the non-duality invariant factors in (4.44). In particular, as shown in [67] the following measure

$$
\hat{\Omega}_{O S V}(p, q)=\int d \phi^{0} d \phi^{1} d \phi^{a}\left|X^{0}\right|^{-2}\left(\operatorname{Im} t^{1}\right)^{\left(n_{V}-4\right) / 2} e^{\mathcal{F}+\pi q^{A} \phi_{A}}
$$

has the remarkable effect of rendering the one-loop approximation to the integral exact, leading to the manifestly duality invariant result

$$
\hat{\Omega}_{O S V}(p, q)=\hat{I}_{1 / 2}\left(S_{0}\right) \sim S_{0}^{-1} \exp \left(S_{0}\right)
$$

Note however that it does not reduce to the constant measure when $p^{0}=0$, and it would therefore spoil agreement with the microscopic counting of DH states. At any rate, irrespective of the choice of measure, it is clear that a duality-invariant measure can no longer be holomorphic for $p^{0} \neq 0$. It would be very desirable to have a deeper understanding of the integration measure implicit in (1.7).

Finally, let us discuss the validity of the assumption that Gromov-Witten instantons can be neglected. If we scale all electric and magnetic charges uniformly by $s$, the entropy $S_{0}$ scales as $s^{2}$, the topological coupling $\lambda \sim 1 /\left|X^{0}\right|$ as $1 / s$ while the Kähler classes $\operatorname{Im} t_{A}$ are fixed. The $\zeta(3)\left(X^{0}\right)^{2}$ term in (4.8) is however comparable to the leading entropy $S_{0}$, so that its effect cannot be neglected. It is therefore necessary to scale the charges $\left(p^{0}, q_{0}\right)$ and $\left(p^{A}, q_{A}\right)$ differently if one is to neglect the Gromov-Witten 
instanton contributions. One option is to take $q_{A} \gg p^{0} \gg\left(q_{0}, p^{A}\right)$. In this regime, the Kähler classes $\operatorname{Im} t_{A}$ grow to infinity as $\sqrt{q_{A} / p^{0}}$, while the coupling $\lambda=\operatorname{Im}\left(1 / X^{0}\right)$ can be made arbitrarily small (in fact zero when $q_{0}=p^{A}=0$ ), so that Gromov-Witten instantons can indeed be neglected.

\section{Microscopic Counting of DH States in $\mathcal{N}=2$ Models}

In this section, we compute the microscopic degeneracies of perturbative DH states in heterotic models with $\mathcal{N}=2$ supersymmetry in four dimensions, which are dual to small black holes in type II string theory compactified on a Calabi-Yau three-fold $\mathcal{X}$. In section 5.1 and 5.2, we discuss the $E_{8} \times E_{8}$ heterotic string compactified on $K_{3}$ with standard, respectively symmetric embedding of the spin connection in the gauge group. In section 5.3, we turn to the FHSV model, which can be viewed as a $\mathcal{N}=2$ analogue of the $\mathcal{N}=4$ models with reduced rank discussed in Section 3. In section 5.4, we obtain a formula which applies to all asymmetric orbifolds of the heterotic string, with $\mathcal{N}=2$ or $\mathcal{N}=4$ supersymmetry.

\subsection{Het $/ K 3 \times T^{2}$ with Standard Embedding}

A simple class of heterotic models with $\mathcal{N}=2$ supersymmetry can be obtained by compactifying the $E_{8} \times E_{8}$ heterotic string on $K 3$, and identifying the spin connection on $K 3$ with the gauge connection for one of the $E_{8}$ factors. The corresponding conformal field theory is most easily constructed at the $\mathbb{Z}_{2}$ orbifold point of $K 3$, where the orbifold generator acts as -1 on the four coordinates of $T^{4}$ (as well as their right-moving superpartners), and as a shift $\left(\frac{1}{2}, \frac{1}{2}, 0^{6}\right)$ in the charge lattice of one of the $E_{8}$ factors. This gives a $\mathcal{N}=2$ model with 628 hypermultiplets transforming as a

$$
4(1,1,1)+8(1,56,1)+(1,56,2)+32(1,1,2)
$$

representation of the $E_{8} \times E_{7} \times S U(2) \times U(1)^{4}$ gauge symmetry. In particular, $N_{V}-N_{H}=$ $388-628=-240$. This model is part of a large network of $\mathcal{N}=2$ vacua which can be reached by a sequence of fundamental or adjoint Higgsing transitions [83]. Of particular interest are the vacua with Abelian gauge symmetry, which can be dual to compactifications of type II string theory on a smooth Calabi-Yau threefold. At a generic point in the moduli space of $K 3$, the $S U(2)$ factor is Higgsed, leaving 10 charged hypers in the 56 of $E_{7}$ and 65 neutral hypermultiplets, for the same value of the index $N_{V}-N_{H}=-240$. Going to the Coulomb branch of $E_{8}$ reduces the gauge symmetry to $E_{7} \times U(1)^{12}$, with index $N_{V}-N_{H}=-480$. Further higgsing the $E_{7}$ factor reduces the gauge symmetry to $U(1)^{12}$ with 492 neutral hypers, a $(12,492)$ model in the 
notation of [83]. This model has been argued to be dual to type II on an hypersurface in $W P_{1,1,12,28,42}^{4}$ [83]. Instead, one may go to the Coulomb branch of $E_{7}$ and obtain a $(19,65)$ model, with 19 vector multiplets and 65 neutral hypers. However, we could also consider going to the Coulomb branch of the original $E_{8} \times E_{7} \times S U(2) \times U(1)^{4}$ gauge symmetry, leading to a $(20,4)$ model with 20 Abelian vectors and 4 neutral hypers.

Let us now consider the degeneracies of $\mathrm{DH}$ states in the original model with unbroken $E_{8} \times E_{7} \times S U(2) \times U(1)^{4}$ gauge symmetry. The helicity generating partition function is obtained straightforwardly as

$$
\begin{aligned}
Z(v, \bar{v})= & \frac{\xi(v) \bar{\xi}(v)}{\tau_{2}|\eta|^{4}} \frac{1}{2} \sum_{h, g=0}^{1} \frac{1}{2} \sum_{a, b=0}^{1}(-1)^{a+b+a b} \frac{\bar{\theta}\left[\begin{array}{c}
\frac{a}{2} \\
\frac{b}{2}
\end{array}\right](v) \bar{\theta}\left[\begin{array}{c}
\frac{a}{2} \\
\frac{b}{2}
\end{array}\right] \bar{\theta}\left[\begin{array}{c}
\frac{a+h}{2} \\
\frac{b+g}{2}
\end{array}\right] \bar{\theta}\left[\begin{array}{c}
\frac{a-h}{2} \\
\frac{b-g}{2}
\end{array}\right]}{\bar{\eta}^{4}} \\
& \frac{Z_{2,2}}{|\eta|^{4}} \frac{\left.Z_{(4,4)}^{o r b} \frac{\frac{h}{2}}{\frac{g}{2}}\right]}{|\eta|^{8}} \times \frac{1}{2} \sum_{\gamma, \delta=0}^{1} \frac{\theta\left[\begin{array}{c}
\frac{\gamma+h}{2} \\
\frac{\delta+g}{2}
\end{array}\right] \theta\left[\begin{array}{c}
\frac{\gamma-h}{2} \\
\frac{\delta-g}{2}
\end{array}\right] \theta^{6}\left[\begin{array}{c}
\frac{\gamma}{2} \\
\frac{\delta}{2}
\end{array}\right]}{\eta^{8}} \times \theta_{E_{8}[1]}
\end{aligned}
$$

where $Z_{(4,4)}^{\text {orb }}$ are the orbifold blocks of the $T^{4} / \mathbb{Z}_{2}$ orbifold,

$$
Z_{(4,4)}\left[\begin{array}{l}
0 \\
0
\end{array}\right]=Z_{4,4}, \quad Z_{(4,4)}\left[\begin{array}{l}
h / 2 \\
g / 2
\end{array}\right]=\frac{2^{4}}{\left|\theta\left[\begin{array}{l}
1-\frac{h}{2} \\
1-\frac{g}{2}
\end{array}\right] \theta\left[\begin{array}{l}
1+\frac{h}{2} \\
1+\frac{g}{2}
\end{array}\right]\right|^{2}}
$$

The sum over spin structures $a, b$ can as usual be performed by using the Riemann identity (B.10). Taking two $\bar{v}$ derivatives and setting $v=\bar{v}=0$, the generating function for the second helicity supertraces is thus

$$
B_{2}=\frac{1}{2} \sum_{h, g}^{\prime} \frac{Z_{2,2} \theta_{E_{8}[1]}}{\tau_{2} \eta^{18} \theta\left[\begin{array}{c}
\frac{1+h}{2} \\
\frac{1+g}{2}
\end{array}\right] \theta\left[\begin{array}{c}
\frac{1-h}{2} \\
\frac{1-g}{2}
\end{array}\right]} \times \sum_{\gamma, \delta=0}^{1} \theta\left[\begin{array}{l}
\frac{\gamma+h}{2} \\
\frac{\delta+g}{2}
\end{array}\right] \theta\left[\begin{array}{l}
\frac{\gamma-h}{2} \\
\frac{\delta-g}{2}
\end{array}\right] \theta^{6}\left[\begin{array}{l}
\frac{\gamma}{2} \\
\frac{\delta}{2}
\end{array}\right]
$$

where the prime indicates that the untwisted, unprojected sector $h=g=0$ has to be omitted.

In order to read off the degeneracies of DH states with prescribed electric charges from this expression, it is convenient to go to a general point in the vector multiplet moduli space. This depends on the phase under consideration:

- in the $(12,492)$ model above, where the gauge symmetry is broken to $U(1)^{12}$, the two factors $Z_{2,2}$ and $\theta_{E_{8}[1]}$ combine into a the partition function $Z_{2,10}$ of the charge lattice $I I_{2,2} \oplus E_{8}$ at a general point in the $S O(2,10) / S O(2) \times S O(10)$ moduli space. Using (B.16) and (B.17), the sum over $h, g$ simplifies to

$$
B_{2}=\frac{Z_{2,10}}{\tau_{2}} \frac{E_{6}}{\eta^{24}}
$$


We thus deduce that the indexed degeneracies of $\mathrm{DH}$ states in this phase are given by the coefficients of

$$
\frac{E_{6}}{\eta^{24}}=\sum_{N=0}^{\infty} \Omega(N) q^{N-1}=\frac{1}{q}-480-\ldots
$$

where $N-1=Q^{2}$. By the Rademacher formula, the degeneracies grow as

$$
\Omega_{2}(Q) \sim \hat{I}_{7}\left(4 \pi \sqrt{Q^{2} / 2}\right)
$$

in agreement with the general prediction (4.27) with $n_{V}=12$.

- in the $(20,4)$ model above, the two factors $Z_{2,2}$ and $\theta_{E_{8}[1]}$ combine with the eight theta series in the numerator into a a vector of partition functions $Z_{2,18} \theta\left[\begin{array}{c}h \\ g\end{array}\right]$ of a lattice

$$
I I_{2,2} \oplus\left(E_{8} \cup\left(E_{8}+\delta\right)\right) \oplus E_{8}
$$

at a general point in its moduli space. The helicity supertrace can be decomposed into four sectors,

$$
\begin{aligned}
\tau_{2} B_{2}= & \frac{Z_{2,18}\left[\begin{array}{l}
0 \\
0
\end{array}\right]+Z_{2,18}\left[\begin{array}{l}
0 \\
1
\end{array}\right]}{2} F_{u}-\frac{Z_{2,18}\left[\begin{array}{l}
0 \\
0
\end{array}\right]-Z_{2,18}\left[\begin{array}{l}
0 \\
1
\end{array}\right]}{2} F_{u} \\
& -\frac{Z_{2,18}\left[\begin{array}{l}
1 \\
0
\end{array}\right]+Z_{2,18}\left[\begin{array}{l}
1 \\
0
\end{array}\right]}{2} F_{+}-\frac{Z_{2,18}\left[\begin{array}{l}
1 \\
0
\end{array}\right]-Z_{2,18}\left[\begin{array}{l}
1 \\
0
\end{array}\right]}{2} F_{-}
\end{aligned}
$$

with

$$
F_{u}=\frac{\theta_{3}^{2} \theta_{4}^{2}}{\eta^{24}} \quad, \quad F_{ \pm}=\frac{\theta_{2}^{2}\left(\theta_{3}^{2} \pm \theta_{4}^{2}\right)}{\eta^{24}}
$$

We thus find that the second helicity supertraces of DH states are enumerated by a different generating function in each conjugacy class of the lattice (5.9). The asymptotics are given by

$$
\begin{aligned}
& \Omega_{2}^{u}(Q) \sim \hat{I}_{11}\left(4 \pi \sqrt{\frac{3}{8} Q^{2}}\right) \\
& \Omega_{2}^{ \pm}(Q) \sim \hat{I}_{11}\left(4 \pi \sqrt{\frac{1}{2} Q^{2}}\right)
\end{aligned}
$$

In particular, the indexed degeneracies in the untwisted sector are exponentially smaller than in the twisted sector. Only the latter coincide with the macroscopic prediction (4.27) with $n_{V}=20$. As we shall see, this is in fact a generic feature of $\mathcal{N}=2$ orbifolds where twisted states can be distinguished from untwisted ones by their charges. 
- Similarly, in the $(19,65)$ model, the $Z_{2,2}$ and $\theta_{E_{8}[1]}$ combine with 7 out of the 8 theta functions in the numerator, into the partition function of a signature $(2,17)$ lattice. The second helicity supertraces in the various sectors are generated by

$$
F_{u}=\frac{\theta_{3}^{2} \theta_{4}^{2}\left(\theta_{3}+\theta_{4}\right)}{\eta^{24}} \quad, \quad F_{ \pm}=\frac{\theta_{2}^{2}\left(\theta_{3}^{2}\left(\theta_{3}+\theta_{2}\right) \pm \theta_{4}^{2}\left(\theta_{4}+\theta_{2}\right)\right)}{\eta^{24}}
$$

Again, using the Rademacher formula, we find agreement with the macroscopic prediction (4.27) with $n_{V}=19$ in the twisted sectors, but not in the untwisted one.

From the above discussion, it is thus clear that the degeneracies of DH states depend on the phase under consideration: as a vector field become massive, black holes which used to carry different charges under this field are no longer distinguishable, leading to an increase of the entropy at fixed charges under massless charges. The total number of states is however conserved. In particular, the same argument as in Section 3.4 shows that the modular weight of the generating function of the second helicity supertrace at fixed charges is directly correlated to the rank of the charge lattice, in agreement with the relation $1-w=\left(n_{V}+2\right) / 2$. The numerical factor in the leading entropy however depends on the sector of consideration, and is typically smaller in the untwisted sector. As we shall discuss in more detail in Section 5.3 in the context of the FHSV model, the absolute degeneracies are however much larger, as the result of large cancellations between massive vector and hypermultiplets.

\subsection{Het $/ K 3 \times T^{2}$ with Symmetric Embedding}

In general, one may construct $\mathcal{N}=2$ heterotic backgrounds by embedding the spin connection into the sum of two rank 2 bundles with $c_{2}=12$ in each $E_{8}$ factor. This admits a simple conformal field theory description as a $\mathbb{Z}_{2} \times \mathbb{Z}_{2}$ orbifold, where the first generator acts as in the standard embedding case, and the second acts purely by a shift along one direction of $T^{4}$ as well as a vector $\left(\frac{1}{2}, \frac{1}{2}, 0^{6}\right)$ in the other $E_{8}$ factor [84]. This results in a model with $E_{7} \times S U(2) \times E_{7} \times S U(2) \times U(1)^{4}$ gauge symmetry and hypermultiplets in

$$
4(56,1 ; 1,1)+4(1,1 ; 56,1)+16(1,2 ; 1,1)+16(1,1 ; 1,2)
$$

This model has $N_{V}-N_{H}=-244$ and can be completely Higgsed into a $(4,244)$ model, dual to type II string theory on $W P_{24}^{1,1,2,8,12}$ with Euler number $\chi=-480$ [83]. The 
helicity partition function at the orbifold point reads

$$
\begin{aligned}
& Z=\frac{\xi(v) \bar{\xi}(v)}{\tau_{2}|\eta|^{4}} \frac{1}{2} \sum_{h, g=0}^{1} \frac{1}{2} \sum_{h^{\prime}, g^{\prime}=0}^{1} \frac{1}{2} \sum_{a, b=0}^{1}(-1)^{a+b+a b} \frac{\bar{\theta}\left[\begin{array}{c}
\frac{a}{2} \\
\frac{b}{2}
\end{array}\right](v) \bar{\theta}\left[\begin{array}{c}
\frac{a}{2} \\
\frac{b}{2}
\end{array}\right] \bar{\theta}\left[\begin{array}{c}
\frac{a+h}{2} \\
\frac{b+g}{2}
\end{array}\right] \bar{\theta}\left[\begin{array}{c}
\frac{a-h}{2} \\
\frac{b-g}{2}
\end{array}\right]}{\bar{\eta}^{4}} \\
& \frac{Z_{2,2}}{|\eta|^{4}} \frac{Z_{(4,4)}^{o r b}\left[\begin{array}{l}
\frac{h}{2} ; \frac{h^{\prime}}{2} ; \frac{g^{\prime}}{2} \\
|\eta|^{8}
\end{array}\right.}{\mid \frac{1}{2}} \sum_{\gamma, \delta=0}^{1} \frac{\theta\left[\begin{array}{l}
\frac{\gamma+h}{2} \\
\frac{\delta+g}{2}
\end{array}\right] \theta\left[\begin{array}{l}
\frac{\gamma-h}{2} \\
\frac{\delta-g}{2}
\end{array}\right] \theta^{6}\left[\begin{array}{l}
\frac{\gamma}{2} \\
\frac{\delta}{2}
\end{array}\right]}{\eta^{8}} \times \frac{1}{2} \sum_{\gamma^{\prime}, \delta^{\prime}=0}^{1} \frac{\theta\left[\frac{\frac{\gamma^{\prime}+h^{\prime}}{2}}{\frac{\delta^{\prime}+g^{\prime}}{2}}\right] \theta\left[\begin{array}{l}
\frac{\gamma^{\prime}-h^{\prime}}{2} \\
\frac{\delta^{\prime}-g^{\prime}}{2}
\end{array}\right] \theta^{6}\left[\begin{array}{l}
\frac{\gamma^{\prime}}{2} \\
\frac{\delta^{\prime}}{2}
\end{array}\right]}{\eta^{8}}
\end{aligned}
$$

In this expression, $Z_{(4,4)}^{\text {orb }}\left[\begin{array}{l}\frac{h}{2} ; \frac{h^{\prime}}{2} \\ \frac{g}{2}\end{array}\right]$ denotes the orbifold block corresponding to a torus $T^{4}$ with twist $(h, g)$ on the 4 directions and shift $\left(h^{\prime}, g^{\prime}\right)$ along, say, the first circle. It is non-vanishing only for $\left(h^{\prime}, g^{\prime}\right)=(0,0)$ or $(h, g)=(0,0)$ or $(h, g)=\left(h^{\prime}, g^{\prime}\right)$. In the latter case, it reduces to the orbifold block $Z_{(4,4)}^{\text {orb } \frac{h}{2}}\left[\begin{array}{l}\frac{h}{2} \\ 2\end{array}\right]$ with twist only. In particular, despite appearances, one may check that the construction is symmetric under exchange of the two $E_{8}$. By using the Riemann identity and (B.16),(B.17), it is again possible to simplify the helicity supertrace into

$$
B_{2}=\frac{Z_{2,2}}{\tau_{2}} \frac{E_{4} E_{6}}{\eta^{24}}
$$

Degeneracies of DH states from this equation can be extracted in the same way as before. The result is simplest in the "maximally Higgsed" phase of the $(4,244)$ model, where the $4 U(1)$ charges correspond to the $T^{2}$ lattice: the generating function for second helicity supertraces of DH states is simply

$$
\frac{E_{4} E_{6}}{\eta^{24}}=\sum_{N=0}^{\infty} \Omega_{2}(N) q^{N-1}=\frac{1}{q}-240+\ldots
$$

with asymptotics

$$
\Omega_{2}(Q) \sim-\hat{I}_{3}\left(4 \pi \sqrt{\frac{1}{2} Q^{2}}\right)
$$

in full agreement with (4.27) for $n_{V}=4$. As before, one may unhiggs this model and increase the rank of the gauge group: in all cases the indexed degeneracies are counted by modular forms of weight $w=-n_{V} / 2$, and agree with (4.27) in the twisted sectors only.

\subsection{The $(2,12)$ FHSV Model}

The FHSV model introduced in [85] is one of the simplest and best understood examples of heterotic/type II duality with $\mathcal{N}=2$ symmetry. On the type II side, it consists of 
an orbifold of Type IIA string theory on $K 3 \times T^{2}$ by the Enriques involution on $K 3$ times a reversal of $T^{2}$ - a close cousin of the $(4,16)$ model. Its dual description may be formulated as a $\mathbb{Z}_{2}$ orbifold of the $E_{8} \times E_{8}$ heterotic string on $T^{4} \times T^{2}$, where the orbifold acts by exchanging the two $E_{8}$ factors $^{23}$. In terms of the momentum lattice

$$
\Gamma_{6,22}=E_{8}(-1) \oplus E_{8}(-1) \oplus I I^{2,2} \oplus I I^{4,4}
$$

the action on the momenta is therefore

$$
g\left|P_{1}, P_{2}, P_{3}, P_{4}\right\rangle=e^{2 \pi i \delta \cdot P_{3}}\left|P_{2}, P_{1}, P_{3},-P_{4}\right\rangle
$$

where $2 \delta$ is the vector $(1,0,1,0) \in I I^{2,2}$ corresponding to the translation by half a period along the first circle.

Diagonalizing the action of $g$ on the oscillators, there are 12 untwisted and 12 twisted left-moving bosons, and 4 twisted and 4 untwisted right-moving $\mathcal{N}=1$ multiplets. Denoting by $\left(\epsilon_{L}, \epsilon_{R}\right)$ the parity of the left and right moving oscillators under the orbifold action, massless states with parity $(+,+)$ correspond to hypermultiplets, while massless states with parity $(-,-)$ correspond to vector multiplets as well as the graviphoton. The massless spectrum therefore consists of 12 hypermultiplets, 11 vectors multiplets and the gravity multiplet, with tree-level moduli space

$$
\frac{S O(4,12, \mathbb{R})}{S O(4) \times S O(12)} \times \frac{S O(2,10, \mathbb{R})}{S O(2) \times S O(10)}
$$

where the first (resp. second) factor is parameterized by the scalar fields in the hypermultiplets (resp. vector multiplets). In fact, it can be shown that there are no quantum corrections to the moduli space metric, and that (5.22) is the exact quantum moduli space, up to global identifications [85]. At any point on the vector multiplet moduli space, a vector $P$ of the lattice (5.20) may be projected into a sum $\Pi_{L}(P)+\Pi_{R}(P)$ in $\mathbb{R}^{22} \oplus \mathbb{R}^{6}$. The linear combination

$$
Z=\Pi_{R}^{1}(P)+i \Pi_{R}^{2}(P)
$$

is the complex central charge $Z$ of the $\mathcal{N}=2$ algebra, while the remaining components $\Pi_{R}^{3,4,5,6}(P)$ are the remnants of the central charges of the $\mathcal{N}=4$ supersymmetry, which is broken by the twist on $T^{4}$. By the same reasoning as in Section 3.3, the 22 left-moving charges $\Pi_{L}^{i}(P)$ decompose into 12 electric charges

$$
Q(P)=\left(P_{1}+P_{2} ; P_{3}\right)
$$

\footnotetext{
${ }^{23}$ We slightly deviate from the action in [85], reversing the coordinates on $T^{4}$, and translating one of the circles in $T^{2}$, which exchanges two $\Gamma_{9,1}$ and reverses a $T^{3}$; the two constructions are expected to be on the same moduli space.
} 
under the gauge fields in the vector multiplets, taking values in the signature $(2,10)$ non-self dual lattice

$$
\Lambda_{0}=E_{8}\left(-\frac{1}{2}\right) \oplus I I^{2,2}
$$

while the remaining 10 are "unphysical charges" under gauge fields which have been projected out.

\section{Untwisted sector}

Now, candidate DH states in the untwisted sector can be constructed as

$$
\mathcal{P}^{ \pm}(\alpha) \cdot\left(\left|P_{1}, P_{2}, P_{3}, P_{4}\right\rangle \pm e^{2 \pi i P_{3} \delta}\left|P_{2}, P_{1}, P_{3},-P_{4}\right\rangle\right) \otimes|\tilde{I}\rangle^{ \pm}
$$

where $\mathcal{P}^{ \pm}(\alpha)$ denotes a generic monomial in the left-moving creation oscillators, with definite parity \pm under the orbifold action $g$, and $|\tilde{I}\rangle^{ \pm}$denotes the right-moving ground states transforming as $8_{v} \oplus 8_{s}$ under the transverse $s o(8)$ rotations in ten-dimensions, with definite parity under $g$. Unlike the $(4,16)$ model, the states $(5.26)$ are BPS only if they saturate the BPS bound $M^{2}=|Z|^{2}$, i.e. $\Pi_{R}^{i}(P)=0$ for $i=3,4,5,6$. More formally, this condition may be written as

$$
\Pi_{R}(P)^{2}=\Pi_{R}(Q(P))^{2}
$$

Note that this condition explicitly depends on the values of the vector multiplet moduli space. For $P_{4} \neq 0$, it is only obeyed on a codimension one submanifold of the vector moduli space, providing an example of the "chaotic BPS states" mentioned in the introduction. As we shall see shortly, these states always come in a vector multiplet / hypermultiplet pair and cancel from the helicity supertrace $\Omega_{2}$. On the other hand, states (5.26) with $P_{4}=0$ are always BPS. In order to enumerate the DH states (5.26), let us introduce the partition function

$$
A_{ \pm}:=\operatorname{Tr}_{\mathcal{H}_{P}} \frac{1}{2}(1 \pm g)=\sum_{I I^{22,6}} q^{\frac{1}{2} \Pi_{L}(P)^{2}} \bar{q}^{\frac{1}{2} \Pi_{R}(P)^{2}} \Pi_{b p s}(P) \frac{1}{2}(1 \pm \Theta(P))
$$

where the projection operator $\Pi_{b p s}(P)$ is $=1$ when $(5.27)$ is satisfied, and $=0$ otherwise, and

$$
\Theta(P)=\delta_{P_{1}, P_{2}} e^{2 \pi i P_{3} \cdot \delta} \delta_{P_{4}, 0}
$$

incorporates the fact that states with $P_{1}=P_{2}, P_{4}=0$ and $e^{2 \pi i \delta P_{3}}=\mp 1$ are dropped out, while those we $P_{1} \neq P_{2}$ or $P_{4} \neq 0$ are counted twice with $1 / 2$ multiplicity, just as in (3.16). Note that $\Pi_{b p s}(P) \Theta(P)=\Theta(P)$. 
In addition, let us introduce the partition functions of the left-moving oscillator excitations $\mathcal{P}^{ \pm}(\alpha)$

$$
B_{ \pm}:=\operatorname{Tr}_{\mathcal{H}_{o s c}} \frac{1}{2}(1 \pm g) q^{L_{0}} \bar{q}^{\bar{L}_{0}}=\frac{1}{2}\left(\frac{1}{\eta^{24}} \pm \frac{2^{6}}{\eta^{6} \vartheta_{2}^{6}}\right):=q^{-1} \sum_{N=0}^{\infty} d_{ \pm}^{u}(N) q^{N}
$$

The partition function for DH states (5.26) with positive parity for the right-moving ground state is thus

$$
Z_{H}=A_{+} B_{+}+A_{-} B_{-}=\frac{1}{2} \frac{1}{\eta^{24}} \sum_{P \in I I^{22,6}} q^{\frac{1}{2} q_{L}^{2}} \bar{q}^{\frac{1}{2} q_{R}^{2}} \Pi_{b p s}(P)+\frac{2^{5}}{\eta^{6} \vartheta_{2}^{6}} \sum_{P \in I I^{22,6}} q^{\frac{1}{2} q_{L}^{2}} \bar{q}^{\frac{1}{2} q_{R}^{2}} \Theta(P)
$$

while, for DH states with negative right-moving parity, it is

$$
Z_{V}=A_{+} B_{-}+A_{-} B_{+}=\frac{1}{2} \frac{1}{\eta^{24}} \sum_{P \in I I^{22,6}} q^{\frac{1}{2} q_{L}^{2}} \bar{q}^{\frac{1}{2} q_{R}^{2}} \Pi_{b p s}(P)-\frac{2^{5}}{\eta^{6} \vartheta_{2}^{6}} \sum_{P \in I I^{22,6}} q^{\frac{1}{2} q_{L}^{2}} \bar{q}^{\frac{1}{2} q_{R}^{2}} \Theta(P)
$$

Generalizing the terminology from the massless sectors, and consistent with the definition in [57], we shall refer to the states of the first type (5.31) as "massive hypermultiplets", and states of the second type (5.32) as "massive vector multiplets". Taking the difference, we find the index

$$
B_{2}=Z_{H}-Z_{V}=\frac{2^{6}}{\eta^{6} \vartheta_{2}^{6}} \sum_{P \in I I^{22,6}} q^{\frac{1}{2} q_{L}^{2}} \bar{q}^{\frac{1}{2} q_{R}^{2}} \Theta(P)
$$

The notation anticipates the fact, to be demonstrated shortly, that this index indeed coincides with second helicity supertrace. The chaotic BPS states thus cancel out from $\Omega_{2}$, leaving only states with $P_{1}=P_{2}$ and $P_{4}=0$. For these states, the indexed degeneracies are thus counted by

$$
\frac{2^{6}}{\eta^{6} \vartheta_{2}^{6}}:=\sum d_{ \pm}^{u}(N) q^{N-1}
$$

Using the Rademacher formula, this is given asymptotically by

$$
d^{u}(N) \sim 2^{-7} \hat{I}_{7}(2 \pi \sqrt{N-1})
$$

Note that the argument of the Bessel function is one half of its usual value, in agreement with the fact that unbroken $\mathcal{N}=4$ supersymmetry in the untwisted sector leads to drastic cancellations in the index $\Omega_{2}$. 


\section{Chaotic BPS states}

While the BPS states cancel from the index $\Omega_{2}$, it is nevertheless of interest to investigate their degeneracies, and exhibit their dependence on the moduli. Let us therefore consider the sum

$$
Z_{H}+Z_{V}=\frac{1}{\eta^{24}} \sum_{P \in I I^{22,6}} q^{\frac{1}{2} q_{L}^{2}} \bar{q}^{\frac{1}{2} q_{R}^{2}} \prod_{b p s}(P)
$$

Now, as in the $(4,16)$ case, we need to rewrite $(5.36)$ as a partition for the physical charges $Q=\left(P_{1}+P_{2} ; P_{3}\right)$. Let us therefore change basis to

$$
\begin{aligned}
& P_{1}+P_{2}=2 S+\mathcal{P} \\
& P_{1}-P_{2}=2 \Delta-\mathcal{P}
\end{aligned}
$$

where $S, \Delta$ both take values in the $E_{8}$ root lattice, and $\mathcal{P}$ is an element of the finite group $Z=\Lambda_{r}\left(E_{8}\right) / 2 \Lambda_{r}\left(E_{8}\right)$. When $\Pi_{b p s}(P)=1$, it is easy to check that

$$
\Pi_{L}(P)^{2}-\Pi_{L}(Q(P))^{2}=-2\left(\Delta-\frac{1}{2} \mathcal{P}\right)^{2}-P_{4}^{2}
$$

This allows to rewrite (5.36) into

$$
Z_{H}+Z_{V}=\frac{1}{\eta^{24}} \sum_{Q \in \Lambda_{0}} q^{\frac{1}{2} \Pi_{L}(Q)^{2}} \bar{q}^{\frac{1}{2} \Pi_{R}(Q)^{2}} \mathcal{F}_{Q}(q)
$$

where $\mathcal{F}_{Q}(q)$ is a sum over "unphysical charges",

$$
\mathcal{F}_{Q}(q)=\sum_{\Delta \in E_{8}[1], P_{4} \in I I^{4,4}} q^{-\left(\Delta-\frac{1}{2} \mathcal{P}\right)^{2}-\frac{1}{2} P_{4}^{2}} \Pi_{b p s}\left(S+\Delta, S-\Delta+\mathcal{P}, P_{3}, P_{4}\right)
$$

Now, for generic moduli $\Pi_{b p s} \neq 0$ only for $\Delta=0, \mathcal{P}=0, P_{4}=0$, so that

$$
\mathcal{F}_{Q}(q)=\delta_{\mathcal{P}, 0}
$$

At special moduli however, $\mathcal{F}_{Q}(q)$ will be a non-trivial theta series. E.g., at the $E_{8} \times$ $E_{8}$ enhanced symmetry point with generic (non-rational) moduli for $I I^{4,4}$, the BPS condition puts $P_{4}=0$, however allows $\Delta, \mathcal{P}$ to be purely leftmoving, leading to

$$
\mathcal{F}_{Q}(q)=\sum_{\Delta \in E_{8}(+1)} q^{\left(\Delta-\frac{1}{2} \mathcal{P}\right)^{2}}=\Theta_{E_{8}[2], \mathcal{P}}(\tau)
$$

The absolute degeneracies of the DH states, counted by

$$
\frac{1}{\eta^{24}} \mathcal{F}_{Q}(q)=:=\sum d_{ \pm}^{a b s}(N) q^{N-\Delta}
$$


will thus have different asymptotics at different points in moduli space,

$$
d^{a b s}(N) \sim \hat{I}_{\nu}(4 \pi \sqrt{N-\Delta})
$$

where the index of the Bessel function will be $\nu=13$ for generic moduli, $\nu=9$ at moduli where (5.43) is valid, and may take other values at different loci. Since the index $\nu$ controls the logarithmic correction to the entropy, the latter would in general depend on the moduli. Note that in all cases, the index $\Omega_{2}$ is exponentially suppressed with respect to the absolute number of BPS states in the untwisted sector.

\section{Twisted sectors}

Let us now briefly turn to the BPS states in the twisted sector of the FHSV model. By a modular transformation, it is easy to see that the electric charges for twisted sectors is

$$
\Lambda_{1}=E_{8}\left(-\frac{1}{2}\right) \oplus\left(I I^{2,2}+\delta\right)
$$

DH states take the form

$$
\mathcal{P}^{ \pm}(\alpha)\left(1 \mp e^{i \pi\left(\frac{1}{2} P^{2}+\left(P_{3}+\delta\right)^{2}\right)}\right)\left|P ; P_{3}+\delta\right\rangle \otimes|t\rangle \otimes|\tilde{s}\rangle
$$

where $|t\rangle$ denotes one of the $2^{6}$ twisted left-moving ground states, and $|\tilde{s}\rangle$ one of the $2^{6} \times 2^{3}$ twisted right-moving ground states, in the Neveu-Schwarz or Ramond sector. DH states with electric charges $Q_{e}=\left(P ; P_{4}\right) \in \Lambda_{1}$ are now enumerated by the partition function

$$
\frac{1}{2}\left(\frac{2^{6}}{\eta^{6} \vartheta_{4}^{6}} \pm \frac{2^{6}}{\eta^{6} \vartheta_{3}^{6}}\right):=\sum \Omega_{ \pm}^{t}(N) q^{N-\Delta_{ \pm}}
$$

where the sign is that of $-e^{i \pi\left(\frac{1}{2} P^{2}+\left(P_{3}+\delta\right)^{2}\right)}$, and $\Delta_{ \pm}= \pm 1 / 4$. Using the Rademacher formula we obtain the asymptotics

$$
\Omega_{ \pm}^{t}(N)=2 \hat{I}_{7}\left(4 \pi \sqrt{N-\Delta_{ \pm}}\right)+\cdots
$$

Comparing with the macroscopic prediction (2.27) with $n_{V}=12$, we find agreement to all orders in inverse charges. As in previous cases, the prescription (1.6) however fails to reproduce the "non-perturbative" corrections in (5.49).

\section{Degeneracies vs. helicity supertraces}

Finally, let us rederive the above results using the formalism of helicity partition functions. By the same reasoning as in (2.36), the helicity partition function of the FHSV 
model reads

$$
\begin{aligned}
Z_{\mathrm{FHSV}}^{H}(v, \bar{v})= & \frac{\xi(v) \xi(\bar{v})}{\tau_{2}|\eta|^{4}} \frac{1}{2} \sum_{h, g} \frac{Z_{4,4}^{\text {orb }}\left[\begin{array}{c}
\frac{h}{2} \\
\frac{g}{2}
\end{array}\right]}{|\eta|^{8}} \frac{Z_{2,2}\left[\begin{array}{c}
\frac{h}{2} \\
\frac{g}{2}
\end{array}\right]}{|\eta|^{4}} \bar{Z}_{c u r}\left[\begin{array}{c}
\frac{h}{2} \\
\frac{g}{2}
\end{array}\right] \\
& \times \frac{1}{2} \sum_{\alpha, \beta}(-1)^{\alpha+\beta+\alpha \beta}\left(\frac{\bar{\theta}\left[\begin{array}{c}
\frac{\alpha}{2} \\
\frac{\beta}{2}
\end{array}\right](\bar{v}) \bar{\theta}\left[\begin{array}{c}
\frac{\alpha}{2} \\
\frac{\beta}{2}
\end{array}\right](0) \bar{\theta}\left[\begin{array}{c}
\frac{\alpha-h}{2}\left[\frac{\beta-g}{2}\right.
\end{array}\right](0) \bar{\theta}\left[\begin{array}{c}
\frac{\alpha-h}{2} \\
\frac{\beta-g}{2}
\end{array}\right](0)}{\bar{\eta}^{4}}\right)
\end{aligned}
$$

where

$$
Z_{2,2}\left[\begin{array}{c}
\frac{h}{2} \\
\frac{g}{2}
\end{array}\right]=\sum_{p \in I I^{2,2}}(-1)^{g(\delta, p))} q^{\frac{1}{2} \Pi_{L}^{2}(p+h \delta)} \bar{q}^{\frac{1}{2} \Pi_{R}^{2}(p+h \delta)}
$$

is the partition function for $T^{2}$ orbifolded by a translation by the order 2 vector $\delta$,

$$
\begin{aligned}
Z_{4,4}^{\text {orb }}\left[\begin{array}{l}
0 \\
0
\end{array}\right] & =\sum_{p \in I I^{4,4}} q^{\frac{1}{2} \Pi_{L}^{2}(p)} \bar{q}^{\frac{1}{2} \Pi_{R}^{2}(p)} \\
Z_{4,4}^{\text {orb }}\left[\begin{array}{c}
\frac{h}{2} \\
\frac{g}{2}
\end{array}\right] & =16 \frac{|\eta|^{12}}{\left|\theta\left[\begin{array}{l}
\frac{1}{2}+\frac{h}{2} \\
\frac{1}{2}+\frac{g}{2}
\end{array}\right] \theta\left[\begin{array}{l}
\frac{1}{2}-\frac{h}{2} \\
\frac{1}{2}-\frac{g}{2}
\end{array}\right]\right|^{2}}, \quad(h, g) \neq(0,0)
\end{aligned}
$$

are the partition functions of the orbifold $T^{4} / \mathbb{Z}_{2}$, and $Z_{\text {cur }}$ is the same as in (3.32). The sum over spin structures can be performed using the Riemann identity, leaving

$$
\begin{aligned}
Z_{\mathrm{FHSV}}^{H}(v, \bar{v})=\frac{1}{2} \sum_{h, g} \frac{\xi(v) \xi(\bar{v})}{\tau_{2}|\eta|^{4}} & \frac{\bar{\theta}_{1}^{2}\left(\frac{\bar{v}}{2}\right) \bar{\theta}\left[\begin{array}{c}
\frac{1}{2}-\frac{h}{2} \\
\frac{1}{2}-\frac{g}{2}
\end{array}\right]\left(\frac{\bar{v}}{2}\right) \bar{\theta}\left[\begin{array}{c}
\frac{1}{2}+\frac{h}{2} \\
\frac{1}{2}+\frac{g}{2}
\end{array}\right]\left(\frac{\bar{v}}{2}\right)}{\bar{\eta}^{4}} \\
& \times \frac{Z_{4,4}^{\text {orb }}\left[\begin{array}{c}
\frac{h}{2} \\
\frac{g}{2}
\end{array}\right]}{|\eta|^{8}} \frac{Z_{2,2}\left[\begin{array}{c}
\frac{h}{2} \\
\frac{g}{2}
\end{array}\right]}{|\eta|^{4}} Z_{\text {cur }}\left[\begin{array}{c}
\frac{h}{2} \\
\frac{g}{2}
\end{array}\right]
\end{aligned}
$$

The leading trace comes at order $v^{2}$, and does not receive any contribution from the $(h, g)=(0,0)$ sector, which has $\mathcal{N}=4$ supersymmetry:

$$
B_{2}=\frac{1}{2 \tau_{2} \eta^{2}} \sum_{(h, g) \neq(0,0)} \frac{16}{\theta\left[\begin{array}{l}
\frac{1}{2}-\frac{h}{2} \\
\frac{1}{2}-\frac{g}{2}
\end{array}\right] \theta\left[\begin{array}{l}
\frac{1}{2}+\frac{h}{2} \\
\frac{1}{2}+\frac{g}{2}
\end{array}\right]} Z_{2,2}\left[\begin{array}{l}
\frac{h}{2} \\
\frac{g}{2}
\end{array}\right] \bar{Z}_{\text {cur }}\left[\begin{array}{c}
\frac{h}{2} \\
\frac{g}{2}
\end{array}\right]
$$

or, equivalently,

$$
B_{2}=\frac{16}{2 \tau_{2}}\left[16 \frac{\theta_{E_{8}[1]}(2 \tau) Z_{2,2}\left[\begin{array}{l}
0 \\
\frac{1}{2}
\end{array}\right]}{\eta^{6} \theta_{2}^{6}}+\frac{\theta_{E_{8}[1]}\left(\frac{\tau}{2}\right) Z_{2,2}\left[\begin{array}{c}
\frac{1}{2} \\
0
\end{array}\right]}{\eta^{6} \theta_{4}^{6}}-\frac{\theta_{E_{8}[1]}\left(\frac{\tau+1}{2}\right) Z_{2,2}\left[\begin{array}{c}
\frac{1}{2} \\
\frac{1}{2}
\end{array}\right]}{\eta^{6} \theta_{3}^{6}}\right]
$$

Identifying the numerators as the partition functions for the lattice $\Lambda_{0}$ and $\Lambda_{1}$, we directly obtain the degeneracies (5.34) and (5.48). The contribution of the chaotic states can be exhibited by looking at the fourth helicity supertrace $\Omega_{4}$. 


\subsection{General $\mathcal{N}=2$ Asymmetric Orbifolds}

Having described the FHSV model in detail, it is not too difficult to compute the degeneracies of DH states for arbitrary asymmetric orbifolds of the heterotic string on the torus $T^{6}$ by a discrete group $\Gamma$. We assume that the constraints level matching and anomaly cancellation are satisfied, which still leaves a large class of possibilities. For simplicity we will focus on the index of DH states in the untwisted sector. We discuss the twisted sector states briefly at the end of this section.

Let $\Gamma$ is a discrete group, with an embedding $R: \Gamma \rightarrow O(22) \times O(6)$. The orbifold group acts by shifts so that the action on momentum vectors is

$$
g|P\rangle=e^{2 \pi i \delta(g) \cdot P}|R(g) P\rangle
$$

In $\mathbb{R}^{22,6}$, with metric $\operatorname{Diag}\left(+1^{22},-1^{6}\right)$ we can diagonalize the rotational part of $R(g)$ as

$$
R(g)=R\left(\theta_{1}(g)\right) \oplus \cdots \oplus R\left(\theta_{11}(g)\right) \oplus R\left(2 \tilde{\theta}_{1}(g)\right) \oplus R\left(\tilde{\theta}_{2}(g)\right) \oplus R\left(\tilde{\theta}_{3}(g)\right)
$$

where $R(\theta)$ is the usual $2 \times 2$ rotation matrix

$$
R(\theta)=\left(\begin{array}{cc}
\cos (2 \pi \theta) & \sin (2 \pi \theta) \\
-\sin (2 \pi \theta) & \cos (2 \pi \theta)
\end{array}\right)
$$

We will sometimes denote $\theta_{j}(g)=r_{j}(g) / N$ where $N=|\Gamma| . \mathcal{N}=2$ supersymmetry requires that $\tilde{\theta}_{1}+\tilde{\theta}_{2}+\tilde{\theta}_{3} \equiv 0 \bmod 1$ so that their exists a complex combination $Z$ of the charges $\Pi_{R}(p)$ which is invariant under $\Gamma$, and which can be identified as the $\mathcal{N}=2$ central charge.

The moduli are the boosts in $O(22,6)$ commuting with the image $R(\Gamma)$. We consider embeddings $\Lambda \subset \mathbb{R}^{22,6}$ of $I I^{22,6}$, and let $\Lambda(g)$ denote the sublattice of vectors fixed by the group element $g$.

DH states in the untwisted sector are contained in the subspace of the 1-string Hilbert space of the form

$$
\mathcal{H}_{\text {osc }, L} \otimes \mathcal{H}_{\text {mom }} \otimes \tilde{\mathcal{H}}_{\text {gnd }}
$$

As already stressed in the FHSV model, even after imposing the level matching constraints, it is still necessary to insert a projection $\Pi_{b p s}$ on states which satisfy the BPS condition $M^{2}=|Z|^{2}$. The DH states can therefore be enumerated by introducing the partition function for the momenta,

$$
\operatorname{Tr}_{\mathcal{H}_{\text {mom }}}\left(U_{2}(g) q^{H} \bar{q}^{\tilde{H}}\right)=\sum_{P \in \Lambda(g)} q^{\frac{1}{2} P_{L}^{2}} \bar{q}^{\frac{1}{2} P_{R}^{2}} e^{2 \pi i \delta(g) P} \Pi_{B P S}(P)
$$


where $U_{i}(g)$ is the representation of $g$ in each of the factor spaces, and for the leftmoving oscillators in the 22 internal directions,

$$
\operatorname{Tr}_{\mathcal{H}_{o s c, L}}\left(U_{1}(g) q^{H} y^{2 J_{3}}\right)=\prod_{j=1}^{11} \frac{-2 \eta(\tau) \sin \pi \theta_{j}(g)}{\theta\left[\begin{array}{l}
\frac{1}{2} \\
\frac{1}{2}+\theta_{j}(g)
\end{array}\right](0 ; \tau)}
$$

where we understand that

$$
\frac{-2 \eta(\tau) \sin \pi \theta_{j}(g)}{\theta\left[\begin{array}{l}
\frac{1}{2} \\
\frac{1}{2}+\theta_{j}(g)
\end{array}\right](0 ; \tau)} \rightarrow \frac{1}{\eta^{2}}
$$

if $\theta_{j}(g)=0$. The contribution of the right-movers as well as the left-moving bosons in the transverse directions can be written as

$$
\frac{1}{2} \sum_{a, b}(-1)^{a+b+a b} \bar{\theta}\left[\begin{array}{c}
\frac{a}{2} \\
\frac{b}{2}
\end{array}\right](\bar{v}) \prod_{i=1,3} \bar{\theta}\left[\begin{array}{c}
\frac{a}{2} \\
\frac{b}{2}-\tilde{\theta}_{i}(g)
\end{array}\right](0) \frac{\sin \pi \tilde{\theta}_{i}}{\bar{\theta}\left[\begin{array}{l}
\frac{1}{2} \\
\frac{1}{2}-\tilde{\theta}_{i}
\end{array}\right](0)}
$$

The sum over spin structures can be carried out by using the generalized Riemann identity (B.13). In the supersymmetric case, this reduces to

$$
\bar{\theta}\left[\begin{array}{c}
\frac{1}{2} \\
\frac{1}{2}
\end{array}\right](\bar{v} / 2) \prod_{i=1,2,3} \bar{\theta}\left[\begin{array}{cc}
\frac{1}{2} & \\
\frac{1}{2}-\tilde{\theta}_{i}(g)
\end{array}\right](\bar{v} / 2) \frac{\sin \pi \tilde{\theta}_{i}}{\bar{\theta}\left[\begin{array}{ll}
\frac{1}{2} \\
\frac{1}{2}-\tilde{\theta}_{i}(g)
\end{array}\right](\bar{v} / 2)}
$$

The ground state contribution is therefore

$$
\left(\sqrt{y}-\frac{1}{\sqrt{y}}\right) \times \prod_{i=1,2,3}\left(\sqrt{y} e^{i \pi \tilde{\theta}_{i}}-\frac{1}{\sqrt{y}} e^{-i \pi \tilde{\theta}_{i}}\right)
$$

In particular, the zeroth and first helicity supertraces vanish, while

$$
\Omega_{2}=2(\sin \pi \tilde{\theta}(g))^{2}
$$

where $\tilde{\theta}_{3}=0, \tilde{\theta}_{1}=-\tilde{\theta}_{2}:=\tilde{\theta} \bmod 1$.

Now let us discuss the charge lattice. Suppose that $k$ pairs of left-moving bosons are fixed for all $g \in \Gamma$. Together with the 2 right-moving directions in the plane of $\tilde{\theta}_{3}$ we have a plane $\mathcal{Q} \subset \mathbb{R}^{22,6}$ of signature $(2 k, 2)$. The vector-multiplet moduli come from the $S O(2 k, 2)$ rotations in this plane. The number of $U(1)$ vector fields is $n_{V}=2 k+2$. The projection of $\Lambda$ into the plane $\mathcal{Q}$ defines the charge lattice (in the untwisted sector) $M_{0}$. Let $\rho: \Lambda \rightarrow M_{0}$ be the projection. States in the untwisted sector are labelled by $P \in I I^{22,6}$ but we only want to discuss degeneracies at a fixed $Q \in M_{0}$. Using the BPS condition $P_{R}^{2}=Q_{R}^{2}$, we may rewrite:

$$
\sum_{P \in \Lambda(g)} q^{\frac{1}{2} P_{L}^{2}} \bar{q}^{\frac{1}{2} P_{R}^{2}} e^{2 \pi i \delta(g) P} \prod_{B P S}(P)=\sum_{Q \in M_{0}} q^{\frac{1}{2} Q_{L}^{2}} \bar{q}^{\frac{1}{2} Q_{R}^{2}} \mathcal{F}_{g, Q}(q)
$$


where

$$
\mathcal{F}_{g, Q}(q)=\sum_{P \in \Lambda(g), \rho(P)=Q} q^{\frac{1}{2}\left(P_{L}^{2}-Q_{L}^{2}\right)} e^{2 \pi i \delta(g) P}
$$

The function (5.69) is actually very simple in many important cases. For example if $\Lambda(g) \subset M_{0}$, which is typical if the fixed space under the group element $g$ coincides with $\mathcal{Q}$ then we simply have $\mathcal{F}_{g, Q}(q)=e^{2 \pi i \delta(g) \cdot Q}$. For this reason it is useful to distinguish between "minimal twists", which leave only the subspace $\mathcal{Q}$ invariant (i.e. $0<\theta_{j}(g)<1$ for $j>k$ ) and nonminimal twists. For nonminimal twists the kernel of $Q_{e l}$ will be nontrivial and $\mathcal{F}_{g, Q}(q)$ will be a theta function.

Putting all this together we find that the degeneracies of untwisted sector BPS states are given by

$$
\begin{gathered}
\Omega_{\mathrm{w}}(Q)=e^{4 \pi Q_{R}^{2}} \int d \tau_{1} q^{\frac{1}{2} Q_{L}^{2}} \bar{q}^{\frac{1}{2} Q_{R}^{2}} \mathcal{Z}_{\omega} \\
\mathcal{Z}_{w}=\frac{1}{N} \sum_{g \in \Gamma} \frac{1}{\eta^{2+2 k}}\left[\prod_{j=1}^{11-k}\left(-2 \sin \pi \theta_{j}(g)\right) \frac{\eta}{\vartheta\left[\begin{array}{c}
\frac{1}{2} \\
\frac{1}{2}+\theta_{j}(g)
\end{array}\right](\mid \tau)}\right] w(g) \mathcal{F}_{g, Q}(q)
\end{gathered}
$$

where $w(g)$ is given by

$$
w(g)= \begin{cases}16 \cos \pi \tilde{\theta}_{1}(g) \cos \pi \tilde{\theta}_{2}(g) \cos \pi \tilde{\theta}_{3}(g) & \mathrm{w}=\mathrm{abs} \\ 2(\sin \pi \tilde{\theta}(g))^{2} & \mathrm{w}=2 \\ \frac{3}{2} & \mathrm{w}=4 \\ \frac{15}{8}\left(2-E_{2}(\tau)\right) & \mathrm{w}=6\end{cases}
$$

This formula is exact. Now let us determine its asymptotics. The general counting function appearing in (5.70) is

$$
K(\tau)=\frac{1}{\eta^{24-3 t}} \prod_{j=1}^{t} \frac{1}{\vartheta\left[\begin{array}{c}
\frac{1}{2} \\
\frac{1}{2}+r_{j} / N
\end{array}\right](0 \mid \tau)}=q^{-1} \sum_{n \geq 0} K_{g}(n) q^{n}
$$

Together with the functions

$$
\frac{1}{\eta^{24-3 t}} \prod_{j=1}^{t} \frac{\frac{1}{2}}{\vartheta\left[\begin{array}{l}
\frac{1}{2}+a_{j} / N \\
\frac{1}{2}+b_{j} / N
\end{array}\right](0 \mid \tau)}
$$

with $0 \leq a_{j}, b_{j}<N$, the function $K$ transforms as a matrix of dimension $N \times N$ and modular weight $w=t-12$ under the congruence subgroup $\Gamma_{0}(N, \mathbb{Z})$ of $\operatorname{Sl}(2, \mathbb{Z})$. In 
order to apply the Rademacher formula, one must diagonalize the $T$ operator in the space spanned by (5.74). After some computation, we find:

$$
K(n)=\left|\Delta_{g}\right|^{1-w} e^{-i \pi \sum_{j}\left(\frac{1}{2}-\theta_{j}\right)} \hat{I}_{1-w}\left(4 \pi \sqrt{(n-1)\left|\Delta_{g}\right|}\right)+\cdots
$$

and

$$
\Delta_{g}:=-1+\frac{1}{2} \sum_{j=1}^{11-k} \theta_{j}(g)\left(1-\theta_{j}(g)\right), \quad 0<\theta_{j}(g)<1
$$

is the ground-state energy in the left-moving sector twisted by $g$. We only get contributions from $g$ such that $\Delta_{g}<0$. In addition, there are non-perturbative corrections of order

$$
\hat{I}_{1-w}\left(4 \pi \sqrt{(n-1)\left|\Delta_{g}+\frac{\ell}{N}\right|}\right)
$$

for $\ell$ such that $\Delta_{g}+\frac{\ell}{N}<0$, and of order

$$
\hat{I}_{1-w}\left(\frac{4}{c} \pi \sqrt{(n-1)\left|\Delta_{g}+\frac{\ell}{N}\right|}\right)
$$

for $c>1$. We conclude that the leading asymptotics for the degeneracies of untwisted DH states from the minimal twists is $(w \neq 6$ here):

$$
\frac{1}{4 N} \sum_{g \in \Gamma, \text { minimal }}^{\prime} w(g) h(g) \prod_{j=1}^{11-k}\left(-2 \sin \pi \theta_{j}(g)\right)\left|\Delta_{g}\right|^{k+2} \hat{I}_{k+2}\left(4 \pi \sqrt{\left|\Delta_{g}\right| \frac{1}{2} Q^{2}}\right)
$$

where

$$
h(g)= \begin{cases}(-1)^{(12-k) / 2} \sin \left(2 \pi \delta(g) Q+\pi \sum_{j} \theta_{j}(g)\right) & \text { keven } \\ (-1)^{(11-k) / 2} \cos \left(2 \pi \delta(g) Q+\pi \sum_{j} \theta_{j}(g)\right) & \text { kodd }\end{cases}
$$

The prime ${ }^{24}$ on the sum indicates we only get contributions from $g$ such that $\Delta_{g}<$ 0. For nonminimal twists there will be similar contributions as described above. In particular the index on the Bessel function will be the same, but (5.76) receives an extra nonnegative contribution from the shift $\delta$, and the coefficient $\left|\Delta_{g}\right|^{k+2}$ is modified (and still positive). In some examples the leading asymptotics is provided by the minimal twists alone.

It is interesting to compare this with the twisted sectors. Since the sector $(1, g)$ always mixes with $(g, 1)$ under modular transformation, and since the oscillator groundstate energy is -1 in the untwisted sector, it is clear that for charges $Q$ corresponding

\footnotetext{
${ }^{24}$ The rest of this section is excerpted from [1].
} 
to states in the twisted sector the asymptotics will grow like

$$
\hat{I}_{k+2}\left(4 \pi \sqrt{\frac{1}{2} Q^{2}}\right)
$$

This is true both for the absolute number of BPS states and for the supertraces. Recall that $k+2=\frac{1}{2}\left(n_{V}+2\right)$ for $\mathcal{N}=2$ compactifications, so we have agreement with (4.28).

There are some interesting general lessons we can draw from the result (5.79). Due to the factor $h(g)$ it is possible that the leading $I$-Bessel functions cancel for certain directions of $Q$. Moreover, a general feature of $\mathcal{N}=2$ compactifications is that $g=1$ does not contribute to $\Omega_{2}$ in (5.79). Then, since $\left|\Delta_{g}\right|<1$ the degeneracies are exponentially smaller in the untwisted sector compared to those of the twisted sector. We have seen explicit examples of this above. In contrast, for $\mathcal{N}=4$ compactifications, the $g=1$ term does contribute to $\Omega_{4}$, which thus has the same growth as in the twisted sector.

One general lesson seems to be that the degeneracies, and even their leading asymptotics can be sensitive functions of the "direction" of $Q$ in charge space. In general it is quite possible that the exact BPS degeneracies and their asymptotics will be subtle arithmetic functions of the charge vector $Q^{25}$. In the physics literature it is taken for granted that there is a smooth function $S: H^{\text {even }}(\mathcal{X}, \mathbb{R}) \rightarrow \mathbb{R}$ so that $S(s Q) \sim \log \Omega_{w}(s Q)$ for $s \rightarrow \infty$, but the true situation might actually be much more subtle. The Rademacher expansion shows that Fourier coefficients of negative weight modular forms have well-defined asymptotics governed by Bessel functions. On the other hand, by contrast, the Fourier coefficients $a_{n}$ of cusp forms of positive weight $k$ have a lot of "scatter" and can only be described by a probability distribution for $a_{n} / n^{(k-1) / 2}$ (see e.g. [87] for an introduction to this subject). As we have remarked above, certain supertraces $d o$ in fact have expressions in terms of positive weight forms and we may expect the asymptotics to be expressed in terms of such probability distributions. It would be very interesting to explore further this dichotomy for the functions $\Omega_{\mathrm{w}}(Q)$.

\section{The Black Hole Partition Function}

In this section we reconsider the black hole partition function, starting with what is known about degeneracies of BPS states, and try to reproduce the structure of the topological string free energy.

\footnotetext{
${ }^{25}$ Such a phenomenon was conjectured based on other considerations in [86].
} 
Since BPS degeneracies, even counted with signs, depend on the background vector multiplet moduli $t^{A}$ (due to jumps at marginal stability), one should specify the background to properly define the partition sum. Furthermore, because the original OSV partition function (1.4) (henceforth denoted as $Z_{0}$ ) does not converge, a regularization needs to be introduced. We will consider

$$
Z_{\alpha}:=\sum_{q} \Omega(p, q) e^{\pi q_{i} \phi^{i}-\pi \alpha H(p, q ; t)}
$$

As we will see below, a suitable and natural choice for $H(p, q ; t)$ is the BPS energy. This introduces additional explicit dependence on $t$, which formally disappears when $\alpha \rightarrow 0$.

For definitiveness we will work in the IIA picture. Since the topological string wave function is defined as an expansion around an infinite radius point, a natural guess is that we should take $\Omega(p, q)$ to count the degeneracies in the corresponding large radius limit. More precisely, we tentatively define

$$
\Omega_{\infty}(p, q ; u):=\lim _{R \rightarrow \infty} \Omega(p, q ; t=i R u)
$$

where $\Omega(p, q ; t)$ is an appropriate index counting the number of BPS states with charge $(p, q)$ on a Calabi-Yau with complexified Kähler form $B+i \omega=t^{A} J_{A}$ and $u$ is a fixed real vector inside the Kähler cone. Note that this definition of the degeneracies still depends on the chosen direction $u$ in the Kähler cone.

For simplicity, we will again mainly consider the case $p^{0}=0$ in what follows. In the $R \rightarrow \infty$ limit, IIA BPS states are then described at vanishing string coupling $g_{s}$ by D4 branes wrapping a divisor $S$, with D2 and D0 branes dissolved into it. For $r$ D4 branes on a rigid divisor $S$, the moduli space $\mathcal{M}$ of this system is the moduli space of semistable rank $r$ coherent sheaves on $S$, with fixed Chern classes $c_{i} \cdot{ }^{26}$ If the divisor is not rigid, $\mathcal{M}$ also includes deformations of $S$. At $g_{s}=0$, BPS ground states are in one to one correspondence with cohomology classes on $\mathcal{M}$. At finite $g_{s}$, some of these may be lifted, but the hyper-vector index $\Omega_{2}$ will remain invariant.

\subsection{Rigid Divisors}

We first consider a class of examples for which the counting is under good control, namely rigid divisors $S$, i.e. $h^{2,0}=h^{1,0}=0$, wrapped by a single D4-brane (so $r=1$ ), with $N$ D0-branes bound to it. We can always construct at least a noncompact CalabiYau $\mathcal{X}$ containing $S$, namely the canonical line bundle over $S$. The simplest example

\footnotetext{
${ }^{26}$ In the case $r=1$, instantons are always pointlike, and $\mathcal{M}$ is simply the Hilbert scheme of $N=c_{2}$ points on $S$. Alternatively, one can turn on a B-field and consider noncommutative instantons, which are smooth even if $r=1$.
} 
is given by $S=\mathbf{P}^{2}$, in which case $\mathcal{X}=O(-3) \rightarrow \mathbf{P}^{2}$. A compact example is given by a D4 wrapping an Enriques surface $S=K 3 / \mathbb{Z}_{2}$ in the FHSV Calabi-Yau threefold $\mathcal{X}=\left(T^{2} \times K 3\right) / \mathbb{Z}_{2}$. These branes are dual to the twisted sector DH states in the dual heterotic model described in section 5.3.

For a rigid divisor $S$, the moduli space $\mathcal{M}_{N}$ is simply the Hilbert scheme of $N$ points on $S$. The number of BPS states $d_{N}:=\operatorname{dim} H^{*}\left(\mathcal{M}_{N}\right)=\chi\left(\mathcal{M}_{N}\right)$ is given by the generating function

$$
\mathcal{Z}(q):=q^{-\chi / 24} \sum_{N} d_{N} q^{N}=\frac{1}{\eta(q)^{\chi}},
$$

where $\chi=h^{1,1}(S)+2$ is the Euler characteristic of $S$. We can also turn on $U(1)$ gauge flux $F$ on $S$, which will induce D2 and D0 brane charge but will otherwise not affect the moduli space. Choosing a basis $C_{I}$ of $H^{2}(S, \mathbb{Z})=\operatorname{Pic}(S)$, which pulls back to a basis of $H_{\text {cpct }}^{1,1}(\mathcal{X})$ if $\mathcal{X}$ is the canonical line bundle over $S$, we get the following net D2 and D0 brane charges

$$
\begin{aligned}
q_{I} & =\int_{S} J_{I} \wedge F \\
q_{0} & =-\left(N-\int_{S} \frac{1}{2} F \wedge F-\frac{\chi}{24}\right) \\
& =-\left(N-\frac{1}{2} C^{I J} q_{I} q_{J}-\frac{\chi}{24}\right) .
\end{aligned}
$$

Here $C^{I J}:=\left(C^{-1}\right)^{I J}$ with $C_{I J}:=C_{I} \cdot C_{J}$. The electric charges can in general have nonintegral shifts:

$$
q_{I} \in \frac{c_{s, I}}{2}+\mathbb{Z}, \quad q_{0} \in-\frac{c_{2}(X) \cdot S}{24}+\mathbb{Z}
$$

The class $c_{s} \in H^{2}(S, \mathbb{Z})$ defines a $\operatorname{spin}^{c}$ structure, and is equivalent, modulo two, to the second Steifel-Whitney class. This charge quantization law follows from the K-theoretic formulation of RR charges and is needed to cancel anomalies, both on the brane worldvolume [88] and on the fundamental string worldsheet [89]. The magnetic charges are given by the homology class of $S$. The Euler characteristic $\chi(S)$ is determined in terms of these magnetic charges only:

$$
\chi=S^{3}+c_{2}(X) \cdot S
$$

Using (6.6), we get:

$$
\Omega_{\infty}(p, q ; u)=d_{N=-q_{0}+\frac{1}{2} C^{I J} q_{I} q_{J}+\frac{\chi}{24}}
$$


Note that in this case, the degeneracies are in fact independent of the choice of $u$. The partition function (1.4) becomes

$$
\begin{aligned}
Z_{0} & =\sum_{N, q_{I}} d_{N} e^{-\pi \phi^{0}\left(-N+\frac{1}{2} C^{I J} q_{I} q_{J}+\frac{\chi}{24}\right)-\pi \phi^{I} q_{I}} \\
& =\mathcal{Z}\left(e^{\pi \phi^{0}}\right) \Theta_{0}\left(\phi^{0}, \phi^{I}\right)
\end{aligned}
$$

where $\mathcal{Z}=1 / \eta^{\chi}$ as in (6.3) and

$$
\Theta_{0}\left(\phi^{0}, \phi^{I}\right):=\sum_{q_{I}} e^{-\pi \phi^{0} \frac{1}{2} C^{I J} q_{I} q_{J}-\pi \phi^{I} q_{I}} .
$$

Convergence of $\mathcal{Z}$ requires $\operatorname{Re} \phi^{0}<0$. On the other hand $C_{I J}$ has signature $\left(1, h^{1,1}-1\right)$. In particular the direction $q_{I} \sim C_{I J} u^{J}$ has positive norm squared. Therefore $\Theta$ is divergent. This signals an instability of the ensemble.

A physically natural way to regularize the partition function is to add an energy dependent Boltzmann factor as in (6.1). More precisely we will take

$$
H(p, q ; u):=\lim _{R \rightarrow \infty}(M(p, q ; i R u)-M(p, 0 ; i R u))
$$

with $M(p, q ; t)$ the mass in string units of a BPS state with charges $p, q$ at the point $t$ in moduli space. We subtracted the $q=0$ energy to get a finite result in the limit $R \rightarrow \infty$. Normalizing $u$ for convenience such that $C_{I J} u^{I} u^{J}:=1$, we get

$$
\begin{aligned}
H & =\lim _{R \rightarrow \infty}\left(\left|q_{0}+i R q_{I} u^{I}-\frac{R^{2}}{2}\right|-\frac{R^{2}}{2}\right) \\
& =-q_{0}+\left(q_{I} u^{I}\right)^{2} .
\end{aligned}
$$

Alternatively we could have obtained this by simply evaluating the $U(1)$ Yang-Mills action on $S$ coupled to D0-branes, to which the DBI action reduces in the limit $R \rightarrow \infty$.

For $-\operatorname{Re} \alpha<\operatorname{Re} \phi^{0}<\operatorname{Re} \alpha$, the modified partition sum (6.1) is convergent:

$$
Z_{\alpha}=\mathcal{Z}\left(e^{\pi\left(\phi^{0}-\alpha\right)}\right) \Theta_{\alpha}\left(\phi^{0}, \phi^{I}\right)
$$

with

$$
\begin{aligned}
\Theta_{\alpha}\left(\phi^{0}, \phi^{I}\right) & :=\sum_{q_{I}} e^{-\frac{\pi}{2} g_{\alpha}^{I J} q_{I} q_{J}-\pi \phi^{I} q_{I}} \\
g_{\alpha}^{I J} & :=\left(\phi^{0}-\alpha\right) C^{I J}+2 \alpha u^{I} u^{J} .
\end{aligned}
$$

The quadratic form $g_{\alpha}^{I J}$ has positive definite real part in the range of $\phi^{0}$ specified above. In particular, the previously problematic direction $q_{I}=C_{I J} u^{J}$ now gives $g_{\alpha}^{I J} q_{I} q_{J}=$ 
$\phi^{0}+\alpha$, which has positive real part. Furthermore, using $\operatorname{det} C^{I J}=(-1)^{h^{1,1}-1}$, we get $\operatorname{det} g_{\alpha}^{I J}=\left(\alpha-\phi^{0}\right)^{h^{1,1}-1}\left(\alpha+\phi^{0}\right)$ and similarly for $\operatorname{det} \operatorname{Re} g_{\alpha}^{I J}$ by replacing the factors by their real parts. Note that this is indeed positive.

Now that we have a convergent expression, we can perform a Poisson resummation on $\Theta$ :

$$
\Theta_{\alpha}\left(\phi^{0}, \phi^{I}\right)=2^{\frac{h^{1,1}}{2}}\left(\alpha-\phi^{0}\right)^{-\frac{h^{1,1}-1}{2}}\left(\alpha+\phi^{0}\right)^{-\frac{1}{2}} \sum_{k^{I}} e^{\frac{\pi}{2} g_{I J}^{\alpha}\left(\phi^{I}+2 i k^{I}\right)\left(\phi^{J}+2 i k^{J}\right)+2 \pi i k^{I} \frac{c_{s, I}}{2}},
$$

with $c_{s, I}$ as in (6.7) and

$$
g_{I J}^{\alpha}=\frac{1}{\phi^{0}-\alpha} C_{I J}+\frac{2 \alpha}{\alpha^{2}-\phi^{0^{2}}} u_{I} u_{J}
$$

with $u_{I}:=C_{I J} u^{J}$.

Finally, we do a modular transformation on $\mathcal{Z}=1 / \eta^{\chi}$ :

$$
\mathcal{Z}\left(e^{\pi\left(\phi^{0}-\alpha\right)}\right)=2^{-\frac{\chi}{2}}\left(\alpha-\phi^{0}\right)^{\frac{\chi}{2}} \mathcal{Z}\left(e^{\frac{4 \pi}{\phi^{0}-\alpha}}\right) .
$$

Combining this with (6.19) and using $\chi=h^{1,1}+2$ and the product formula for $\eta$ gives

$$
\begin{aligned}
Z_{\alpha}= & \frac{\alpha-\phi^{0}}{2}\left(\frac{\alpha-\phi^{0}}{\alpha+\phi^{0}}\right)^{1 / 2} \prod_{n}\left(1-e^{\frac{4 \pi n}{\phi^{0}-\alpha}}\right)^{-\chi} \\
& \times \sum_{k_{I}} \exp \left(-\frac{\pi \chi}{6\left(\phi^{0}-\alpha\right)}+\frac{\pi}{2} g_{I J}^{\alpha}\left(\phi^{I}+2 i k^{I}\right)\left(\phi^{J}+2 i k^{J}\right)+2 \pi i k^{I} \frac{c_{s, I}}{2}\right)
\end{aligned}
$$

Inverting (6.1), we thus get

$$
\Omega_{\infty}(p, q)=\int_{-i}^{i} d \phi^{0} \int_{-i}^{i} d \phi^{I} e^{\pi q_{i} \phi^{i}+\alpha H(p, q ; u)} Z_{\alpha}\left(\phi^{0}, \phi^{I} ; u\right) .
$$

Note that the sum over $k^{I}$ in (6.22) can be dropped by extending the domain of the integrals over $\phi^{I}$ to $(-i \infty,+i \infty)$. Furthermore, by definition, the expression is independent of $\alpha$ (and $u$ ), so we can take the limit $\alpha \rightarrow 0$, which formally gives

$$
\Omega_{\infty}(p, q)=\int_{-i}^{i} d \phi^{0} \int_{-i \infty}^{i \infty} d \phi^{I} f\left(\phi^{0}\right) e^{\pi\left(-\frac{S^{3}+c_{2} \cdot S}{6 \phi^{0}}+\frac{1}{2 \phi^{0}} C_{I J} \phi^{I} \phi^{J}+q_{0} \phi^{0}+q_{I} \phi^{I}\right)},
$$

where we used (6.8) to express $\chi$ in terms of the magnetic charge given by $S$, and we defined

$$
f\left(\phi^{0}\right):=\frac{\phi^{0}}{2 i} \prod_{n}\left(1-e^{\frac{4 \pi n}{\phi^{0}}}\right)^{-\chi(S)} .
$$


The integral (6.24) is somewhat formal, because of the oscillatory Gaussian integral and the infinite product in (6.25) which is not well behaved on the imaginary axis. From the above we know however that it is unambiguously defined as the limit $\alpha \rightarrow 0+$ of the same integral with replacements

$$
\phi^{0} \rightarrow \phi^{0}-\alpha, \quad C_{I J} \rightarrow C_{I J}-\frac{2 \alpha}{\alpha+\phi^{0}} u_{I} u_{J}
$$

\section{Comparison with the topological string}

Comparing to (4.11), we see that the quantity in the exponential in (6.24) is exactly the perturbative part of the free energy derived from the topological string amplitude. ${ }^{27}$ Let us elaborate a bit on the term quadratic in $\phi$. On the topological string side, it corresponds to the term $C_{A B} \phi^{A} \phi^{B}$ with $C_{A B}:=C_{A B C} p^{C}$ and $A, B: 1, \ldots, b_{2}(\mathcal{X})$. The integers $C_{A B}$ give the intersection products of the pullbacks of a basis of $H^{1,1}(\mathcal{X})$ to $S$. Since $h^{2,0}(S)=0$, these pullbacks span all of $H^{2}(S)$, and $C_{A B}$ has rank $b_{2}(S)$. Note that in general this can be smaller than $b_{2}(\mathcal{X})$. After a suitable change of $\phi$ variables, we can thus rewrite the term $C_{A B} \phi^{A} \phi^{B}$ as $C_{I J} \phi^{I} \phi^{J}$, with $I=1, \ldots, b_{2}(S)$ and $C_{I J}$ the intersection form on $H^{2}(S)$ as defined before. The remaining $\phi^{A}$ with $A=b_{2}(S)+1, \ldots, b_{2}(\mathcal{X})$ no longer appear in the perturbative part of the supergravity free energy, and the latter thus reduces to the "S-local" expression in the exponential in (6.24).

Clearly however, at least for this simple class of wrapped D-branes, the GromovWitten part of the topological string free energy is not generated by the BPS partition sum. In particular there is no $\phi^{I}$ dependence apart from the quadratic term, whereas typically the Gromov-Witten series is a very complicated function of the $\phi^{I}$. The infinite product in $f\left(\phi^{0}\right)$ looks somewhat like the infinite products appearing in the Gopakumar-Vafa formula (4.10) for the topological string wave function but actually does not seem to have any obvious interpretation in this context. It depends only on $\phi^{0}$, so it would have to come from the homologically trivial worldsheet sector, which however has a quite different form.

At large $\left|q_{0}\right|$, the integral is well approximated by a saddle point evaluation, and at the saddle point, $\phi^{0}$ will be small and negative, so the infinite product in $f\left(\phi^{0}\right)$ will be exponentially close to 1 . Dropping this factor will therefore merely give exponentially small deviations from the exact answer. This is not so however for the additional $\phi^{0}$

\footnotetext{
${ }^{27}$ Note that our basis of charges indeed has a cubic prepotential, as assumed in (4.11). In such a basis the electric charges of D4-brane states will in general have nonintegral shifts. By substituting $q_{0} \rightarrow n_{0}-\left(c_{2} \cdot S\right) / 24$ and $q_{I} \rightarrow n_{I}+c_{s, I} / 2$ in accordance with the quantization shifts $(6.7)$, we get the free energy in an integral basis. The additional terms proportional to the charge shifts correspond to the linear resp. quadratic terms in the prepotential which indeed generally appear for such a basis.
} 
factor, which does not appear on the topological string side of the conjecture. On the other hand, we just saw that $\Delta b:=b_{2}(\mathcal{X})-b_{2}(S)$ of the $\phi^{A}$ decouple from the perturbative part of the free energy on the topological string side. Moreover these $\phi^{A}$ have a natural periodicity $\phi^{0}$, so integrating them out would naturally give an additional factor $\left(\phi^{0}\right)^{\Delta b}$. In the FHSV example with $S$ an Enriques surface, we have $\Delta b=1$, hence for large $q_{0}$ this procedure leads to complete agreement between microscopic and perturbative macroscopic answers, up to exponentially suppressed terms. This agrees with what we found in section 5.3, and will hold similarly for more general $K 3$-fibered examples.

However, more generally, it need not be true that $\Delta b=1$. One can easily imagine simple divisors $S$ of low $b_{2}(S)$ embedded in a Calabi-Yau $\mathcal{X}$ with large $b_{2}(\mathcal{X})$. In those cases the discrepancy by a factor of $\phi^{0}$ cannot be compensated by taking into account the decoupled integrals. Perhaps a better prescription would therefore be to simply discard all decoupled integrals, restricting only to the "local" variables, and adding a factor $\phi^{0}$ by hand as a universal measure contribution.

It should be noted though that the rigid divisors we are considering here are not ample (ample divisors have typically many moduli and always give a nondegenerate $\left.D_{A B}\right)$. This implies that the attractor point computed from the perturbative part of the prepotential will not lie inside the Kähler cone, so there is a priori no reason whatsoever to expect any agreement between the microscopic degeneracies and the macroscopic prediction computed with only the perturbative part of the prepotential. The fact that (modulo the small issue of the $\phi^{0}$ factor) there $i s$ nevertheless agreement to all orders in $1 /\left|q_{0}\right|$ is therefore very remarkable.

\section{A remark on $k$-shifts}

The expression (6.22) for the partition function contains a sum over shifts labelled by $k$. This gives $Z$ the required periodicity in $\phi^{A}$. It is easy to see that this sum over $k$-shifts will be a general feature of the partition function if one assumes the integral form of the conjecture,

$$
\Omega(p, q) \sim \int d \phi e^{\pi q \cdot \phi+\mathcal{F}(\phi)},
$$

where the integrations are over the imaginary axis. Indeed, substituting this in (1.4) gives

$$
Z_{0} \sim \int d \phi^{\prime} e^{\mathcal{F}\left(\phi^{\prime}\right)} \sum_{q} e^{2 \pi i q\left(\frac{\phi-\phi^{\prime}}{2 i}\right)}
$$

Assuming $q$ is quantized as $q=\bar{q}+s$ with $\bar{q} \in \mathbb{Z}$, and using $\sum_{\bar{q}} e^{2 \pi i \bar{q} x}=\sum_{k} \delta(x-k)$, 
this gives

$$
Z_{0} \sim \sum_{k \in \mathbb{Z}} e^{\mathcal{F}(\phi+2 i k)+2 \pi i s \cdot k}
$$

which is precisely the $k$-shift structure found above. Note however that (6.22) does not contain a sum over $k^{0}$. This is related to the fact that the integrand in (6.24) is periodic in $\phi^{0}$ and that the $\phi^{0}$ integral is over one period. In principle, by modifying the integrand, one could try to convert this again to an integral over the entire imaginary axis, and then the expression of $Z$ derived from this modified integrand will also contain as sum over $k^{0}$. In practice, such modifications do not affect the $1 / N$ expansion of (6.24), since this depends only on the neighborhood of the saddle point.

\section{2 $K 3$ Divisors in $K 3 \times T^{2}$}

The rigid examples considered thus far are rather special. In particular we only considered the rank $r=1$ case. To see if perhaps we reproduce more of the topological string amplitude in some large $r$ limit, we apply the same idea to our basic example, $X=K 3 \times T^{2}$, with $r=p^{1}$ coincident D4-branes on $S=K 3$.

The degeneracies are now given by

$$
\Omega_{\infty}\left(p, q_{0}, q_{1}, \vec{q} ; u\right)=\delta_{q_{1}, 0} p_{24}(N), \quad N=1-q_{0} p^{1}+\frac{1}{2} \vec{q}^{2} .
$$

where $\vec{q} \in I I^{19,3}$. The factor $\delta_{q_{1}, 0}$ arises because in the $R \rightarrow \infty$ limit, there are no bound states of D-branes with 6 mutually Dirichlet-Neumann directions. This also fits with the fact that there is no attractor point when $q_{1} \neq 0$. At finite $R$ and sufficiently large $\vec{q}$ or $B$-field, BPS states with nonzero $q_{1}$ may appear [90-93]. The supergravity solutions corresponding to those states will be multicentered, decaying at some point when $R \rightarrow \infty$ [94-96]. Since we are considering the strict limit $R \rightarrow \infty$ here, we do not need to consider these.

The computation of the partition function is similar to the previous subsection. There is one new element: in solving the level matching condition for $q_{0}$, we must ensure integrality of $q_{0}=-\frac{1}{p^{1}}\left(N-1-\vec{q}^{2} / 2\right)$. This is easily achieved by inserting a projector:

$$
Z_{0}=\sum_{N, \vec{q}} \frac{1}{p^{1}} \sum_{k^{0}=0}^{p^{1}-1} e^{-2 \pi i \frac{k^{0}}{p^{1}}\left(N-1-\vec{q}^{2} / 2\right)} p_{24}(N) e^{\pi \frac{\phi^{0}}{p^{1}}\left(N-1-\vec{q}^{2} / 2\right)-\pi \vec{\phi} \cdot \vec{q}} .
$$

As before, this sum is divergent, but can again be regularized. ${ }^{28}$ We will not do this in detail, but use its existence as justification for the formal manipulations in the following.

${ }^{28}$ Because the D2 charge lattice now has 3 positive norm squared directions, spanned by $(\omega, \operatorname{Re} \Omega, \operatorname{Im} \Omega)$, where $\omega$ is the Kähler form and $\Omega$ the holomorphic 2-form on K3, the regularization will involve $\Omega$ as well as $\omega$. This is special to cases with $\mathcal{N}=4$ supersymmetry. 
Carrying out the sum over $N$, we get

$$
Z_{0}=\frac{1}{p^{1}} \sum_{k^{0}=0}^{p^{1}-1} \eta^{-24}\left(e^{\pi\left(\phi^{0}-2 i k^{0}\right) / p^{1}}\right) \sum_{\vec{q}} e^{-\frac{\pi}{p^{1}}\left(\phi^{0}-2 i k^{0}\right) \vec{q}^{2}-\pi \vec{\phi} \cdot \vec{q}} .
$$

Finally, performing a modular transformation on the Dedekind function and a Poisson resummation over $\vec{q}$, we obtain the main formula of this subsection:

$$
Z_{0}=\frac{1}{2 i\left(p^{1}\right)^{2}} \sum_{k^{0}=0}^{p^{1}-1}\left(\phi^{0}-2 i k^{0}\right) \sum_{\vec{k} \in I I^{3,19}} \exp \left(\frac{\pi}{2} \frac{p^{1}(\vec{\phi}-2 i \vec{k})^{2}}{\phi^{0}-2 i k^{0}}-\log \eta^{24}\left(e^{\frac{4 \pi p^{1}}{\phi^{0}-2 i k^{0}}}\right)\right) .
$$

This is very similar to what we found in section 6.1 , with the addition of a finite sum over shifts of $\phi^{0}$. We can also write this in integral form:

$$
\Omega(p, q)=\frac{1}{2 i\left(p^{1}\right)^{2}} \int_{-i p^{1}}^{i p^{1}} d \phi^{0} \int_{-i \infty}^{i \infty} d \vec{\phi} \phi^{0} \exp \left(\frac{\pi}{2} \frac{p^{1} \vec{\phi}^{2}}{\phi^{0}}-\log \eta^{24}\left(e^{\frac{4 \pi p^{1}}{\phi^{0}}}\right)+q_{0} \phi^{0}+\vec{q} \cdot \vec{\phi}\right)
$$

which should be compared to the conjectured

$\Omega(p, q) \stackrel{?}{=} \int d \phi^{0} d \phi^{1} d \vec{\phi} \exp \left(\frac{\pi}{2} \frac{p^{1} \vec{\phi}^{2}}{\phi^{0}}-\log \eta^{24}\left(e^{\frac{2 \pi\left(p^{1}+i \phi^{1}\right)}{\phi^{0}}}\right)-\log \eta^{24}\left(e^{\frac{2 \pi\left(p^{1}-i \phi^{1}\right)}{\phi^{0}}}\right)+q_{0} \phi^{0}+\vec{q} \cdot \vec{\phi}\right)$.

This is similar to the exact expression (6.34), but clearly not quite the same. Working formally, we can fourier expand the $1 / \eta^{24}$ functions in (6.35) and integrate $\phi^{1}$ over $\left(0, \phi^{0}\right)$. This gives

$$
\Omega(p, q) \stackrel{?}{=} \int d \phi^{0} d \vec{\phi} \phi^{0} \exp \left(\frac{\pi}{2} \frac{p^{1} \vec{\phi}^{2}}{\phi^{0}}+\log \sum_{n}\left(p_{24}(n)\right)^{2} e^{\frac{4 \pi p^{1}}{\phi^{0}}(n-1)}+q_{0} \phi^{0}+\vec{q} \cdot \vec{\phi}\right) .
$$

Unfortunately, this differs from (6.34) in that $p_{24}(N)$ appears squared here, but not so in the expansion of the $1 / \eta^{24}$ in (6.34).

In conclusion, at least for the case of $K 3$, we see that considering arbitrary rank still does not fully reproduce the topological amplitude.

\subsection{General Case and Monodromy Invariance}

Despite the arbitrary rank, the K3 case is still somewhat degenerate, insofar as it does not correspond to a regular, "large" black hole. Unfortunately, exact counting of microstates of general D4-D2-D0 systems is considerably harder than the cases we considered so far. However, some information about the form of the partition function can be obtained purely from monodromy invariance, where the monodromy under consideration is around large radius, i.e. integral shifts of the B-field. 
To make this precise, let us first review the general relation of electric and magnetic charges to microscopic quantities. The magnetic charge of $r$ coincident D4-branes wrapped around a divisor $S=m^{A} J_{A}$ is $p^{A}=r m^{A}$. The D0 and D2 electric charges $q_{0}$ and $q_{A}$ corresponding to a rank $r$ coherent sheaf with Chern classes $c_{i}$ are given by

$$
\begin{aligned}
q_{A} & =\int_{S} \iota^{*} J_{A} \wedge\left(c_{1}+\frac{1}{2} r c_{s}\right) \\
q_{0} & =-\left(\frac{\Delta}{2 r}-\int_{S} \frac{1}{2 r}\left(c_{1}+\frac{1}{2} r c_{s}\right)^{2}-\frac{1}{24} r \chi(S)\right) \\
\text { with } \Delta & :=\int_{S} 2 r c_{2}+(1-r) c_{1}^{2} .
\end{aligned}
$$

Here $c_{s}$ is again the Chern class of a $\operatorname{spin}^{c}$ structure on $S$, as discussed earlier, $\chi(S)=$ $S^{3}+c_{2}(X) \cdot S$ is the Euler characteristic of $S, \iota^{*}$ is the pullback map to $S$, and $\Delta$ is the Bogomolov discriminant $[97,98]$. For semistable sheaves $\Delta \geq 0$. When $\Delta$ is sufficiently large, the dimension of the sheaf moduli space is $d=\Delta-\left(r^{2}-1\right) \chi\left(\mathcal{O}_{S}\right)$. As before, the electric charges as defined above in general may have nonintegral shifts. More precisely

$$
q_{A} \in \frac{r c_{s, A}}{2}+\mathbb{Z}, \quad q_{0} \in-\frac{r c_{2}(X) \cdot S}{24}+\mathbb{Z}
$$

One universal feature of the D-brane moduli space $\mathcal{M}$ in the limit $R \rightarrow \infty$ is that it is invariant under monodromy of the charges around large radius. These monodromies can be thought of as induced by shifts $B \rightarrow B+n^{A} J_{A}, n^{A} \in \mathbb{Z}$. At the level of sheaves, this corresponds to tensoring with a line bundle, which maps

$$
c_{1} \rightarrow c_{1}+r n^{A} \iota^{*} J_{A}
$$

and leaves $\Delta$ invariant. The $\omega$-stability condition for sheaves is that every subsheaf of rank $r^{\prime}$ and first Chern class $c_{1}^{\prime}$ must satisfy $c_{1}^{\prime} \cdot \omega / r^{\prime}<c_{1} \cdot \omega / r$ (with $\omega$ the Kähler form), so monodromy does not affect this condition and the BPS spectrum is preserved. ${ }^{29}$ The monodromy action on the charges is

$$
q_{0} \rightarrow q_{0}+q_{A} n^{A}+\frac{r}{2} C_{A B} n^{A} n^{B}, \quad q_{A} \rightarrow q_{A}+r C_{A B} n^{B}
$$

where $C_{A B}:=C_{A B C} m^{C}$. The shift by $c_{s}$ in (6.37) is precisely such that the change in $q_{0}$ is guaranteed to be integral. Invariance of the degeneracies $\Omega_{\infty}(p, q ; u)$ under this transformation implies that they will only depend on the monodromy invariant $\Delta$ and

\footnotetext{
${ }^{29}$ This is only true for physical BPS states when $R=\infty$. At finite $R$, I-stability is the proper physical criterion rather than $\omega$-stability [91,92]. For any arbitrarily large but fixed $R, \Pi$-stability becomes qualitatively different from $\omega$-stability for sufficiently large charges $q_{A}$.
} 
a label $s$ in a finite set giving the value of the D2 charge modulo monodromies. The number of monodromy inequivalent classes grows with $P$. Nevertheless, at any finite value of $P$, monodromy invariance constrains the $\phi^{A}$-dependence of the partition sum to be given by a finite sum of theta functions.

To see this, let us consider the unregularized partition function (1.4) and work formally (this can again be regularized and justified as before). We write $q_{A}=$ $s_{A}+r C_{A B} n^{B}$, where $s_{A}$ parametrizes D2-charges modulo monodromies and $n^{B} \in \mathbb{Z}$. Assuming $C_{A B}$ is nondegenerate, ${ }^{30}$ the $s_{A}$ take values in a finite set $\mathcal{Q}$ of order $\left|\operatorname{det} r C_{A B}\right|$. Correspondingly, we decompose the partition sum as

$$
Z_{0}=\sum_{q_{0}, s_{A}, n^{A}} \Omega_{\infty}\left(p, q_{0}, s_{A}+r C_{A B} n^{B} ; u\right) e^{-\pi\left(\phi^{0} q_{0}+\phi^{A} s_{A}+\phi^{A} r C_{A B} n^{B}\right)}
$$

Using monodromy invariance and shifting $q_{0}$ then gives

$$
\begin{aligned}
Z_{0} & =\sum_{q_{0}, s_{A}, n^{A}} \Omega_{\infty}\left(p, q_{0}-s_{A} n^{A}-\frac{r}{2} C_{A B} n^{A} n^{B}, s_{A} ; u\right) e^{-\pi\left[\phi^{0} q_{0}+\phi^{A} s_{A}+\phi^{A} r C_{A B} n^{B}\right]} \\
& =\sum_{q_{0}^{\prime}, s_{A}, n^{A}} \Omega_{\infty}\left(p, q_{0}^{\prime}, s_{A} ; u\right) e^{-\pi\left[\phi^{0} q_{0}^{\prime}+\phi^{0}\left(s_{A} n^{A}+\frac{r}{2} C_{A B} n^{A} n^{B}\right)+\phi^{A} s_{A}+\phi^{A} r C_{A B} n^{B}\right]} \\
& =\sum_{s \in \mathcal{Q}} \mathcal{Z}_{s}\left(\phi^{0}\right) \Theta_{s}\left(\phi^{0}, \phi^{A}\right)
\end{aligned}
$$

In the last line we defined

$$
\begin{aligned}
\mathcal{Z}_{s}\left(\phi^{0}\right) & :=\sum_{q_{0}} \Omega_{\infty}\left(p, q_{0}, s_{A} ; u\right) e^{-\pi \phi^{0} q_{0}} \\
\Theta_{s}\left(\phi^{0}, \phi^{A}\right) & :=\sum_{n^{I}} e^{-\pi\left[\frac{\phi^{0} r}{2} C_{A B} n^{A} n^{B}+n^{A}\left(s_{A} \phi^{0}+r C_{A B} \phi^{B}\right)+\phi^{A} s_{A}\right]} .
\end{aligned}
$$

Thus we see that the $\phi^{A}$ dependence of the partition sum is given by a finite sum of theta functions $\Theta_{s}$. After a modular transformation, this could be brought in a form analogous to $(6.33)$, but in any case, the $\phi^{A}$ dependence will still be given by a finite sum of theta functions. Brought in integral form, analogous to (6.34), this will give a finite sum of Gaussian functions in the $\phi^{A}$.

\footnotetext{
${ }^{30}$ This is guaranteed if $S$ is very ample. In other cases, such as the $K 3$ example studied above, it may happen that the $q_{A}$ induced on $S$ take values in a linear subspace of the full charge space (because $\iota^{*}: H^{2}(X) \rightarrow H^{2}(S)$ fails to be injective), so the quadratic form $C_{A B}$ will be degenerate. In such cases, we can restrict to that linear subspace, generically the restricted quadratic form will be invertible, and essentially all of what follows goes through. If the restricted quadratic form is still degenerate, there will be an infinite number of monodromy inequivalent classes, and the discussion needs to be changed somewhat.
} 
This should be compared to the $\phi^{A}$ dependence of the topological string amplitude squared, which is given by an intricate series of instanton corrections determined by a typically infinite set of Gromov-Witten invariants, or by an infinite product determined by the likewise infinite set of BPS invariants. It is of course very unlikely that this will in general match a finite set of Gaussian functions.

However, when $P=r S \rightarrow \infty$, the number of these Gaussian terms goes to infinity. Therefore, this result does not contradict the weaker form of the conjecture, i.e. asymptotically for $P \rightarrow \infty$.

Clearly, more results on exact BPS degeneracies of D-brane systems corresponding to large black holes would be very useful to make further progress using the approach of this section.

\section{Conclusion}

In this work, we have studied the detailed degeneracies of small black holes, using their dual description as perturbative heterotic BPS states. The comparison with the macroscopic Bekenstein-Hawking-Wald entropy including the leading $R^{2}$ corrections, and assuming a mixed statistical ensemble, shows a remarkable agreement to all orders in an asymptotic expansion in inverse charges, in a large set of models with $\mathcal{N}=2$ and $\mathcal{N}=4$ supersymmetry. At the same time, we found apparent discrepancies in special models, where however the macroscopic computation is not under good control since the moduli are attracted to the boundary of the Kähler cone. It would be very interesting to generalize our analysis to the case of "large" black holes, with nonvanishing entropy at tree-level, where these effects do not occur. This would require improving our understanding of the effective conformal field theory which describes the micro-states. It would also be interesting to understand the relation with other approaches which postulate a statistical ensemble [15-18], or more drastically trade the singular black hole geometry with a sum over smooth geometries [99].

Acknowledgments : It is a pleasure to thank E. Diaconescu, B. Florea, J. Kappeli, E. Kiritsis, C. Kounnas, Y. Oz, A. Sen, A. Strominger, C. Vafa, E. Verlinde and B. de Wit for valuable discussions. BP is grateful to the the Theory Group at Rutgers University for hospitality during part of this work. The work of FD and GM is supported in part by DOE grant DE-FG02-96ER40949. The work of BP is supported in part by the European Research Network MRTN-CT-2004-512194. 


\section{Appendices}

\section{A. The Rademacher Expansion}

Here we state briefly the Rademacher expansion. For more details and information see [43].

Suppose we have a "vector-valued nearly holomorphic modular form," i.e., a collection of functions $f_{\mu}(\tau)$ which form a finite-dimensional unitary representation of the modular group of weight $w<0$. Under the standard generators we have

$$
\begin{aligned}
& f_{\mu}(\tau+1)=e^{2 \pi i \Delta_{\mu}} f_{\mu}(\tau) \\
& f_{\mu}(-1 / \tau)=(-i \tau)^{w} S_{\mu \nu} f_{\nu}(\tau)
\end{aligned}
$$

We assume the $f_{\mu}(\tau)$ have no singularities for $\tau$ in the upper half plane, except at the cusps $\mathbf{Q} \cup i \infty$. We may assume they have an absolutely convergent Fourier expansion

$$
f_{\mu}(\tau)=q^{\Delta_{\mu}} \sum_{m \geq 0} F_{\mu}(m) q^{m} \quad \mu=1, \ldots, r
$$

with $F_{\mu}(0) \neq 0$ and that the $\Delta_{\mu}$ are real. We wish to give a formula for the Fourier coefficients $F_{\mu}(m)$.

Define:

$$
\hat{I}_{\nu}(z)=-i(2 \pi)^{\nu} \int_{\epsilon-i \infty}^{\epsilon+i \infty} t^{-\nu-1} e^{\left(t+z^{2} /(4 t)\right)} d t=2 \pi\left(\frac{z}{4 \pi}\right)^{-\nu} I_{\nu}(z)
$$

for $\operatorname{Re}(\nu)>0, \epsilon>0$, where $I_{\nu}(z)$ is the standard modified Bessel function of the first kind.

Then we have:

$$
\begin{aligned}
F_{\nu}(n)= & \sum_{c=1}^{\infty} \sum_{\mu=1}^{r} c^{w-2} K \ell(n, \nu, m, \mu ; c) \sum_{m+\Delta_{\mu}<0} F_{\mu}(m) \\
& \left|m+\Delta_{\mu}\right|^{1-w} \hat{I}_{1-w}\left[\frac{4 \pi}{c} \sqrt{\left|m+\Delta_{\mu}\right|\left(n+\Delta_{\nu}\right)}\right] .
\end{aligned}
$$

The coefficients $K \ell(n, \nu, m, \mu ; c)$ are generalized Kloosterman sums, defined as

$$
\mathrm{Kl}(n, \nu ; m, \mu ; c):=\sum_{0<d<c ; d \wedge c=1} e^{2 \pi i \frac{d}{c}\left(n+\Delta_{\nu}\right)} M\left(\gamma_{c, d}\right)_{\nu \mu}^{-1} e^{2 \pi i \frac{a}{c}\left(m+\Delta_{\mu}\right)}
$$

where

$$
\gamma_{c, d}=\left(\begin{array}{cc}
a & (a d-1) / c \\
c & d
\end{array}\right)
$$


is an element of $S l(2, \mathbb{Z})$ and $M(\gamma)$ its matrix representation. For $c=1$ in particular, we have:

$$
K \ell(n, \nu, m, \mu ; c=1)=S_{\nu \mu}^{-1}
$$

The series (A.5) is convergent. Moreover the asymptotics of $I_{\nu}$ for large $\operatorname{Re}(z)$ is given by

$$
I_{\nu}(z) \sim \frac{e^{z}}{\sqrt{2 \pi z}}\left[1-\frac{(\mu-1)}{8 z}+\frac{(\mu-1)\left(\mu-3^{2}\right)}{2 !(8 z)^{2}}-\frac{(\mu-1)\left(\mu-3^{2}\right)\left(\mu-5^{2}\right)}{3 !(8 z)^{3}}+\ldots\right],
$$

where $\mu=4 \nu^{2}$.

\section{B. Modular Cornucopia}

In this section, we collect definitions and useful identities of modular forms. The Jacobi theta function is defined by ${ }^{31}$

$$
\theta\left[\begin{array}{l}
a \\
b
\end{array}\right](v \mid \tau)=\sum_{n \in \mathbb{Z}} q^{\frac{1}{2}(n-a)^{2}} e^{2 \pi i(v-b)(n-a)},
$$

where $a, b$ are real and $q=e^{2 \pi i \tau}$. It satisfies the modular properties

$$
\begin{gathered}
\theta\left[\begin{array}{l}
a \\
b
\end{array}\right](v \mid \tau+1)=e^{-i \pi a(a-1)} \theta\left[\begin{array}{l}
a \\
a+b-\frac{1}{2}
\end{array}\right](v \mid \tau) \\
\theta\left[\begin{array}{l}
a \\
b
\end{array}\right]\left(\frac{v}{\tau} \mid-\frac{1}{\tau}\right)=e^{2 i \pi a b+i \pi \frac{v^{2}}{\tau}} \theta\left[\begin{array}{l}
a \\
b
\end{array}\right](v \mid \tau)
\end{gathered}
$$

The Jacobi-Erderlyi theta functions are the values at half periods,

$$
\theta_{1}(z \mid \tau)=\theta\left[\begin{array}{c}
\frac{1}{2} \\
\frac{1}{2}
\end{array}\right](z \mid \tau), \quad \theta_{2}(z \mid \tau)=\theta\left[\begin{array}{c}
\frac{1}{2} \\
0
\end{array}\right](z \mid \tau), \quad \theta_{3}(z \mid \tau)=\theta\left[\begin{array}{l}
0 \\
0
\end{array}\right](z \mid \tau), \quad \theta_{4}(z \mid \tau)=\theta\left[\begin{array}{l}
0 \\
\frac{1}{2}
\end{array}\right](z \mid \tau)
$$

In particular,

$$
\theta_{1}(v / \tau,-1 / \tau)=i \sqrt{-i \tau} e^{i \pi v^{2} / \tau} \theta_{1}(v, \tau)
$$

The Dedekind $\eta$ function is defined as

$$
\eta(\tau)=q^{\frac{1}{24}} \prod_{n=1}^{\infty}\left(1-q^{n}\right)
$$

It satisfies the modular property

$$
\eta\left(-\frac{1}{\tau}\right)=\sqrt{-i \tau} \eta(\tau)
$$

\footnotetext{
${ }^{31}$ This differs from the definition in [56] by a factor of 2 in the characteristics.
} 
It is related to the Jacobi-Erderlyi theta functions by the identities

$$
\begin{aligned}
\left.\frac{\partial}{\partial v} \theta_{1}(v)\right|_{v=0} & =2 \pi \eta^{3}(\tau) \\
\theta_{2}(0 \mid \tau) \theta_{3}(0 \mid \tau) \theta_{4}(0 \mid \tau) & =2 \eta^{3}
\end{aligned}
$$

The Riemann identity allows to carry out sums over spin structures,

$$
\left.\frac{1}{2} \sum_{a, b=0}^{1}(-1)^{a+b+a b} \prod_{i=1}^{4} \theta \theta_{\frac{b}{2}}^{\frac{a}{2}}\right]\left(v_{i}\right)=-\prod_{i=1}^{4} \theta_{1}\left(v_{i}^{\prime}\right),
$$

where

$$
\begin{aligned}
v_{1}^{\prime}=\frac{1}{2}\left(-v_{1}+v_{2}+v_{3}+v_{4}\right) \quad, \quad v_{2}^{\prime}=\frac{1}{2}\left(v_{1}-v_{2}+v_{3}+v_{4}\right), \\
v_{3}^{\prime}=\frac{1}{2}\left(v_{1}+v_{2}-v_{3}+v_{4}\right) \quad, \quad v_{4}^{\prime}=\frac{1}{2}\left(v_{1}+v_{2}+v_{3}-v_{4}\right) .
\end{aligned}
$$

A generalized form holds provided $\sum_{i} h_{i}=\sum_{i} g_{i}=0$ :

$$
\left.\frac{1}{2} \sum_{a, b=0}^{1}(-1)^{a+b+a b} \prod_{i=1}^{4} \theta\left[\frac{a+h_{i}}{2}\right] \frac{b+g_{i}}{2}\right]\left(v_{i}\right)=-\prod_{i=1}^{4} \theta\left[\frac{\frac{1-h_{i}}{2}}{\frac{1-g_{i}}{2}}\right]\left(v_{i}^{\prime}\right)
$$

The Jacobi and Dedekind function satisfy the following "doubling identities":

$$
\begin{gathered}
\theta_{2}(\tau)=\frac{2[\eta(2 \tau)]^{2}}{\eta(\tau)}, \quad \theta_{3}(\tau)=e^{\frac{i \pi}{12}} \frac{\left[\eta\left(\frac{\tau+1}{2}\right)\right]^{2}}{\eta(\tau)}, \quad \theta_{4}(\tau)=\frac{\left[\eta\left(\frac{\tau}{2}\right)\right]^{2}}{\eta(\tau)} \\
\theta_{2}(2 \tau)=\frac{1}{\sqrt{2}} \sqrt{\theta_{3}^{2}(\tau)-\theta_{4}^{2}(\tau)}, \quad \theta_{3}(2 \tau)=\frac{1}{\sqrt{2}} \sqrt{\theta_{3}^{2}(\tau)+\theta_{4}^{2}(\tau)} \\
\theta_{4}(2 \tau)=\sqrt{\theta_{3}(\tau) \theta_{4}(\tau)}, \quad \eta(2 \tau)=2^{-2 / 3} \theta_{2}^{2 / 3}(\tau)\left(\theta_{3}(\tau) \theta_{4}(\tau)\right)^{1 / 6} \\
\theta_{2}(\tau / 2)=\sqrt{2 \theta_{2}(\tau) \theta_{3}(\tau)}, \quad \theta_{3}(\tau / 2)=\sqrt{\theta_{3}^{2}(\tau)+\theta_{2}^{2}(\tau)} \\
\theta_{4}(\tau / 2)=\sqrt{\theta_{3}^{2}(\tau)-\theta_{2}^{2}(\tau)}, \quad \eta(\tau / 2)=2^{-1 / 6} \theta_{4}^{2 / 3}(\tau)\left(\theta_{2}(\tau) \theta_{3}(\tau)\right)^{1 / 6} \\
\theta_{4}\left(\frac{\tau+1}{2}\right)=\sqrt{\theta_{4}^{2}(\tau)-i \theta_{2}^{2}(\tau)}, \quad \eta\left(\frac{\tau+1}{2}\right)=2^{-1 / 6} e^{\frac{i \pi}{8}} \sqrt{2 \theta_{2}(\tau) \theta_{4}(\tau)}, \quad \theta_{3}^{2 / 3}(\tau)\left(\theta_{2}(\tau) \theta_{4}(\tau)\right)^{1 / 6} \\
\eta(2 \tau) \eta(14)
\end{gathered}
$$


Another convenient set of modular forms are the Eisenstein series,

$$
\begin{gathered}
E_{2}=\frac{12}{i \pi} \partial_{\tau} \log \eta=1-24 \sum_{n=1}^{\infty} \frac{n q^{n}}{1-q^{n}}, \\
E_{4}=\frac{1}{2}\left(\vartheta_{2}^{8}+\vartheta_{3}^{8}+\vartheta_{4}^{8}\right)=1+240 \sum_{n=1}^{\infty} \frac{n^{3} q^{n}}{1-q^{n}} \\
E_{6}=\frac{1}{2}\left(\vartheta_{2}^{4}+\vartheta_{3}^{4}\right)\left(\vartheta_{3}^{4}+\vartheta_{4}^{4}\right)\left(\vartheta_{4}^{4}-\vartheta_{2}^{4}\right)=1-504 \sum_{n=1}^{\infty} \frac{n^{5} q^{n}}{1-q^{n}} .
\end{gathered}
$$

$E_{4}$ and $E_{6}$ have modular weight 4 and 6 , and generate the ring of modular forms under $S l(2, \mathbb{Z}) . E_{2}$ is not a proper modular form as it transforms inhomogeneously under the modular group.

It is also useful to define the following function

$$
\xi(v)=\prod_{n=1}^{\infty} \frac{\left(1-q^{n}\right)^{2}}{\left(1-q^{n} e^{2 \pi i v}\right)\left(1-q^{n} e^{-2 \pi i v}\right)}=\frac{\sin \pi v}{\pi} \frac{\vartheta_{1}^{\prime}}{\vartheta_{1}(v)} .
$$

which often appears in generating functions of helicity supertraces. Its first $v$-derivatives at $v=0$ are

$$
\xi(0)=1, \quad \xi^{\prime}(0)=0, \quad \xi^{(2)}(0)=-\frac{\pi^{2}}{3}\left(1-E_{2}\right)
$$

\section{Counting $J=0$ DH States in the $(4,24)$ Model}

Although there do not exist regular BPS spherically symmetric spinning black hole solutions of the tree-level supergravity, heterotic DH states in general may carry angular momentum $J$. It is conceivable that these states correspond to multi-centered black holes, or require the inclusion of higher derivative corrections. In this section, we examine the degeneracies of DH states in Het $/ T^{6}$ with a prescribed value of the angular momentum $J$, and show that the restriction to DH states with $J=0$ leads to different subleading corrections for the entropy as compared to the case where all values of the angular momentum are summed over. This suggests that the statistical ensemble implicit in the Bekenstein-Hawking-Wald entropy allows for arbitrary fluctuations of the angular momentum, at vanishing potential $\Omega$ conjugate to $J$.

Let us start by recalling that the angular momentum of $\mathrm{DH}$ states arises from bosonic and fermionic oscillators in the two non-compact coordinates transverse to the light-cone. Right-moving oscillators map one state to another in the same supersym-

metry multiplet (unless they break the BPS property), so the angular momentum of the highest weight state of a given multiplet arises from left-movers only. Introducing a 
parameter $v$ conjugate to the left-moving helicity $J_{3}^{L}$ of the highest weight, the partition function of DH states is given by

$$
\Omega_{4}(v, q)=\operatorname{Tr}\left[\left(J_{3}^{R}\right)^{4} e^{i \pi v J_{3}^{L}} q^{L_{0}} \bar{q}^{\bar{L}_{0}}\right]=\frac{3 \sin \pi v}{\eta^{21}(\tau) \theta_{1}(v ; \tau)}
$$

Using $\theta_{1}^{\prime}(0)=2 \pi \eta^{3}$, this reproduces (2.41) when $v=0$. The right-hand side of this equation may be viewed (C.1) as the character of the trivial representation of affine $S l(2)_{k}$, and decomposed into contributions of fixed $U(1)$ charge using a generalization of the Kac-Peterson formula,

$$
\chi_{S l(2)_{k}}^{0}=\frac{2 q^{1 / 8} \sin \pi v}{\theta_{1}(v, \tau)}=\sum_{m=-\infty}^{\infty} e^{2 \pi i m v} q^{-\frac{m^{2}}{k}} \hat{c}_{m}^{j=0}(\tau)
$$

The $S l(2, \mathbb{R})$ level $k$ string functions $\hat{c}_{m}^{j=0}$ have been computed in [100,101] and read

$$
\hat{c}_{m}^{j=0}=\frac{q^{|m|+\frac{m^{2}}{k}}}{q^{-1 / 8} \eta^{3}}\left(1+\left(1+q^{|m|}\right) \sum_{n=1}^{\infty}(-1)^{n} q^{\frac{1}{2}\left[n^{2}+(2|m|+1) n-2|m|\right]}\right)
$$

(Notice that the level $k$ does not affect the spectrum, except for an overall shift.) This allows us to extract the partition function of states of given left-moving helicity $m=h_{L}>0$,

$$
Z_{h e l}(m, q)=\frac{3}{\eta^{21}} q^{-\frac{1}{8}-\frac{m^{2}}{k} \hat{c}_{m}^{j=0}}=\frac{3}{2 \eta^{24}}\left(q^{m}+\left(1+q^{m}\right) \sum_{n=1}^{\infty}(-1)^{n} q^{\frac{1}{2}\left[n^{2}+(2 m+1) n\right]}\right)
$$

Since each multiplet of spin $J$ contributes $2 J+1$ states with $m$ ranging from $-J$ to $J$, one can obtain the partition function of given angular momentum $J$ by

$$
Z_{\text {spin }}(J, q)=Z_{\text {hel }}(J, q)-Z_{\text {hel }}(J+1, q)
$$

Using (C.3), this may be rewritten as

$$
Z_{\text {spin }}(J, q)=\frac{3}{2 \eta^{24}}\left(1+q^{J}+\left(2+q^{J}+q^{-(J+1)}\right) \sum_{n=1}^{\infty}(-1)^{n} q^{\frac{1}{2}\left[n^{2}+(2 J+1) n\right]}\right)
$$

In particular, for $J=0$, we find $Z_{\text {spin }}(0, q)=\frac{1}{\eta^{24}} \cdot S_{0}(q)$ where

$$
S_{0}(q)=1-3 q+q^{2}+3 q^{3}-q^{5}-3 q^{6}+\cdots=2+(1+3 q) \sum_{n=1}^{\infty}(-1)^{n} q^{\frac{1}{2} n(n+1)-1}
$$


Working out (C.5) at low levels, we obtain (up to an overall factor of $3 / 2$ )

$$
\begin{aligned}
& J=0: q^{-1}+21+253 q+2255 q^{2}+16446 q^{3}+\ldots \\
& J=1: \quad 1+22 q+276 q^{2}+2552 q^{3}+\ldots \\
& J=2: \quad q+22 q^{2}+277 q^{3}+\ldots \\
& J=3: \quad q^{2} \quad+22 q^{3} \quad+\ldots
\end{aligned}
$$

reproducing the total partition function,

$$
Z(q)=\sum_{J=0}^{\infty}(2 J+1) Z_{\text {spin }}(J, q)=\frac{3}{2}\left(\frac{1}{q}+24+324 q+3200 q^{2}+25650 q^{3}+\ldots\right)
$$

(notice that the degeneracy of each Regge trajectory stabilize to a constant as the excitation level becomes large, 1,22,277,2576,19574,...)

Let us now extract the asymptotics of the degeneracies $\Omega(J ; N)$. Although the string functions have modular weight $-1 / 2$, their behavior under modular transformations is ill-understood, so that the Rademacher formula does not apply directly. Relatedly, the partition function (C.1) is not a weak Jacobi form. Nevertheless, we may try and obtain the leading asymptotics by saddle point methods ${ }^{32}$. Using (C.1) and (C.5), we have

$$
\Omega_{\text {spin }}(N, J)=4 i \int_{i L-\frac{1}{2}}^{i L+\frac{1}{2}} d \tau \int_{0}^{1} d v e^{-2 \pi i(N-1) \tau+2 i \pi\left(J+\frac{1}{2}\right) v} \frac{\sin ^{2} \pi v}{\eta^{21}(\tau) \theta_{1}(v, \tau)}
$$

In this expression, the range of the $\tau$ integration is chosen such that it corresponds to a small circle around the origin in the $q=e^{2 \pi i \tau}$ variable. Using the modular properties (B.5) and (B.7) and approximating $\eta(-1 / \tau) \sim \tilde{q}^{1 / 24}, \theta_{1}(v / \tau,-1 / \tau) \sim 2 q^{1 / 8} \sin (\pi v / \tau)$ with $\tilde{q}=e^{-2 \pi i / \tau}$, we obtain

$$
\Omega_{\text {spin }}(N, J) \sim-2 i \int_{i L-\frac{1}{2}}^{i L+\frac{1}{2}} d \tau(-i \tau)^{11} \int_{0}^{1} d v e^{-2 \pi i(N-1) \tau+\frac{2 \pi i}{\tau}+i \pi \frac{v^{2}}{\tau}+2 i \pi\left(J+\frac{1}{2}\right) v} \frac{\sin ^{2}(\pi v)}{\sin (\pi v / \tau)}
$$

Rescaling the variables as

$$
\tau=\frac{x}{\sqrt{N-1}}, \quad v=-\frac{J+1 / 2}{\sqrt{N-1}}+x y
$$

the integral becomes

$$
\begin{aligned}
& \Omega_{\text {spin }}(N, J) \sim-2 i \int d x d y\left(\frac{-i x}{\sqrt{N-1}}\right) \frac{\sin ^{2}\left[\pi x\left(y-\pi \frac{J+\frac{1}{2}}{\sqrt{N-1}}\right)\right]}{\cos (\pi y \sqrt{N-1})} \\
& e^{2 \pi i \sqrt{N-1}\left(-x+\frac{1}{x}+x \frac{\left(J+\frac{1}{2}\right)^{2}}{N-1}+\frac{1}{2} x y^{2}\right)}
\end{aligned}
$$

\footnotetext{
${ }^{32}$ Degeneracies of strings with prescribed angular momentum were studied in [31], for a different scaling of the charges.
} 
Unfortunately, saddle point methods do not seem to apply straightforwardly, due to the large oscillations in the denominator. For $J=0$, we find numerically that

$$
\Omega_{\text {spin }}(N, J=0) \sim N^{-33 / 4} e^{4 \pi \sqrt{N}}
$$

which is suppressed by $O\left(N^{-3 / 2}\right)$ compared to the all- $J$ result (2.14). In particular, the success of the OSV conjecture appears to depend on choosing an ensemble where the angular momentum is free to fluctuate at zero conjugate potential $\Omega=0$.

\section{Other $\operatorname{Het}\left(4, n_{V}\right)$ and $\operatorname{Het}\left(2, n_{V}\right)$ Models}

In this appendix, we discuss other heterotic orbifold models with $\mathcal{N}=4$ or $\mathcal{N}=$ 2 supersymmetry and reduced rank. We start with a different construction of the $(4,16)$ model discussed in Section 3.3, now based on the $S O(32)$ heterotic string in ten dimensions. This construction can be easily generalized to produce models with rank 12,10 and 9.

\section{Another $H e t(4,16)$ model}

As explained in [49], the heterotic string at a point of enhanced symmetry $S O(16) \times$ $S O(16)$ may be obtained by orbifolding the $S O(32)$ heterotic string compactified on $S_{1}$ by a $\mathbb{Z}_{2}$ action $g_{1}$, which shifts the $U(1)$ charges of 8 out of the 16 left-moving bosons by half a unit, as well as acts by a translation by half a period along the circle $S_{1}$. The partition function is most easily written by decomposing the level 1 characters of $S O(32)$ under $S O(16) \times S O(16)$, using the general formula

$$
\begin{aligned}
O_{2 n} & =O_{n} O_{n}+V_{n} V_{n} \\
V_{2 n} & =O_{n} V_{n}+V_{n} O_{n} \\
S_{2 n} & =S_{n} S_{n}+C_{n} C_{n} \\
C_{2 n} & =S_{n} C_{n}+C_{n} S_{n}
\end{aligned}
$$

relating the level 1 characters of $S O(2 n)$ in the O,V,S,C conjugacy classes to the level 1 characters of $S O(n)$. Either of them are expressed in terms of free fermion partition functions,

$$
\left(\begin{array}{c}
O_{n} \\
V_{n}
\end{array}\right)=\frac{1}{2}\left(\theta_{3}^{n / 2} \pm \theta_{4}^{n / 2}\right), \quad\left(\begin{array}{c}
S_{n} \\
C_{n}
\end{array}\right)=\frac{1}{2}\left(\theta_{2}^{n / 2} \pm\left(-i \theta_{1}\right)^{n / 2}\right)
$$

In this fashion, the partition function for the Narain lattice $\Gamma_{1,17}$ at the $S O(16) \times S O(16)$ point can be written as

$$
\frac{1}{4} Z_{1,1}\left[\begin{array}{l}
0 \\
0
\end{array}\right]\left(\sum_{a, b=0,1} \theta^{16}\left[\begin{array}{l}
a \\
b
\end{array}\right]\right)+\frac{1}{2} Z_{1,1}\left[\begin{array}{l}
0 \\
\frac{1}{2}
\end{array}\right] \theta_{3}^{8} \theta_{4}^{8}+\frac{1}{2} Z_{1,1}\left[\begin{array}{c}
\frac{1}{2} \\
0
\end{array}\right] \theta_{2}^{8} \theta_{3}^{8}+\frac{1}{2} Z_{1,1}\left[\begin{array}{c}
\frac{1}{2} \\
\frac{1}{2}
\end{array}\right] \theta_{2}^{8} \theta_{4}^{8}
$$


or, decomposing into the various sectors,

$$
\begin{array}{r}
Z_{+}^{0}\left(O_{16} O_{16}+S_{16} S_{16}\right)+Z_{-}^{0}\left(V_{16} V_{16}+C_{16} C_{16}\right) \\
+Z_{+}^{\frac{1}{2}}\left(O_{16} S_{16}+S_{16} O_{16}\right)+Z_{-}^{\frac{1}{2}}\left(V_{16} C_{16}+C_{16} V_{16}\right)
\end{array}
$$

where

$$
Z_{ \pm}^{h / 2}=\frac{1}{2}\left(Z_{1,1}\left[\begin{array}{l}
h / 2 \\
0
\end{array}\right] \pm Z_{1,1}\left[\begin{array}{l}
h / 2 \\
\frac{1}{2}
\end{array}\right]\right)
$$

denotes the projected lattice sum in the $h$-th twisted sector. Compactifying this model further on $S_{1}^{\prime} \times T^{4}$ to four dimensions, we may now take a further $\mathbb{Z}_{2}$ freely acting orbifold which exchanges the two $S O(16)$ factors and acts as a translation by half a period on $S_{1}^{\prime}$ : the untwisted, unprojected sector contributes

$$
\begin{gathered}
\frac{Z_{6,6}\left[\begin{array}{c}
00 \\
00
\end{array}\right]+Z_{6,6}\left[\begin{array}{c}
00 \\
\frac{1}{2} 0
\end{array}\right]}{4}\left(O_{16} O_{16}+S_{16} S_{16}\right)+\frac{Z_{6,6}\left[\begin{array}{c}
00 \\
00
\end{array}\right]-Z_{6,6}\left[\begin{array}{c}
00 \\
\frac{1}{2} 0
\end{array}\right]}{4}\left(V_{16} V_{16}+C_{16} C_{16}\right) \\
+\frac{Z_{6,6}\left[\begin{array}{c}
\frac{1}{2} 0 \\
00
\end{array}\right]+Z_{6,6}\left[\begin{array}{c}
\frac{1}{2} 0 \\
\frac{1}{2} 0
\end{array}\right]}{4}\left(O_{16} S_{16}+S_{16} O_{16}\right) \frac{Z_{6,6}\left[\begin{array}{c}
\frac{1}{2} 0 \\
00
\end{array}\right]-Z_{6,6}\left[\begin{array}{c}
\frac{1}{2} 0 \\
\frac{1}{2} 0
\end{array}\right]}{4}\left(V_{16} C_{16}+C_{16} V_{16}\right)
\end{gathered}
$$

while the untwisted, projected sector reads

$$
\frac{Z_{6,6}\left[\begin{array}{c}
00 \\
0 \frac{1}{2}
\end{array}\right]+Z_{6,6}\left[\begin{array}{c}
00 \\
\frac{1}{2} \frac{1}{2}
\end{array}\right]}{4}\left[O_{16}(2 \tau)+S_{16}(2 \tau)\right]+\frac{Z_{6,6}\left[\begin{array}{c}
00 \\
0 \frac{1}{2}
\end{array}\right]-Z_{6,6}\left[\begin{array}{c}
00 \\
\frac{1}{2} \frac{1}{2}
\end{array}\right]}{4}\left[V_{16}(2 \tau)+C_{16}(2 \tau)\right]
$$

The twisted, unprojected sector can be obtained by modular S transformation,

$$
\frac{Z_{6,6}\left[\begin{array}{l}
0 \frac{1}{2} \\
00
\end{array}\right]+Z_{6,6}\left[\begin{array}{c}
\frac{1}{2} \frac{1}{2} \\
00
\end{array}\right]}{4}\left[O_{16}\left(\frac{\tau}{2}\right)+S_{16}\left(\frac{\tau}{2}\right)\right]+\frac{Z_{6,6}\left[\begin{array}{c}
0 \frac{1}{2} \\
00
\end{array}\right]-Z_{6,6}\left[\begin{array}{c}
\frac{1}{2} \frac{1}{2} \\
00
\end{array}\right]}{4}\left[V_{16}\left(\frac{\tau}{2}\right)+C_{16}\left(\frac{\tau}{2}\right)\right]
$$

and finally, the twisted, projected sector is obtained by a further $\mathrm{T}$ transformation,

$$
\begin{gathered}
\frac{Z_{6,6}\left[\begin{array}{c}
0 \frac{1}{2} \\
0 \frac{1}{2}
\end{array}\right]+Z_{6,6}\left[\begin{array}{c}
\frac{1}{2} \frac{1}{2} \\
\frac{1}{2} \frac{1}{2}
\end{array}\right]}{4}\left[O_{16}\left(\frac{\tau+1}{2}\right)+S_{16}\left(\frac{\tau+1}{2}\right)\right] \\
+\frac{Z_{6,6}\left[\begin{array}{c}
0 \frac{1}{2} \\
0 \frac{1}{2}
\end{array}\right]-Z_{6,6}\left[\begin{array}{c}
\frac{1}{2} \frac{1}{2} \\
\frac{1}{2} \frac{1}{2}
\end{array}\right]}{4}\left[V_{16}\left(\frac{\tau+1}{2}\right)+C_{16}\left(\frac{\tau+1}{2}\right)\right]
\end{gathered}
$$

In order to obtain the degeneracies of states with given electric charges under the diagonal $S O(16)$, we need to change basis and rewrite the product of level 1 characters in (D.9) into a sum of products of $D_{8}=S O(16)$ level 2 theta functions with characteristics. One may check that the finite group $D_{8} / 2 D_{8}$ decomposes into 7 orbits, with 
respective length $1,1,1,1,56,140,56$ corresponding to (i) the orbit of the origin (ii) the orbits of one half the highest weights of the (level 1) V,S,C representations (iii) the orbits of the highest weights of the (level 2) $\Lambda_{2}, \Lambda_{4}, \Lambda_{6}$ representations, of dimension 120 , 1820 and 8008. In cases (i) and (ii), the theta function with characteristics is simply obtained by doubling the argument of the level 1 case, i.e.

$$
\theta_{D_{8}[2] ; O}(\tau)=O_{16}(2 \tau), \quad \theta_{D_{8}[2] ; V}(\tau)=V_{16}(2 \tau), \text { etc }
$$

while, in case (iii), an explicit computation shows that

$$
\theta_{D_{8}[2] ; 120}(\tau)=\frac{1}{2} \theta_{2}^{2} \theta_{3}^{6}(2 \tau), \quad \theta_{D_{8}[2] ; 1820}(\tau)=\frac{1}{2} \theta_{2}^{4} \theta_{3}^{4}(2 \tau), \quad \theta_{D_{8}[2] ; 8008}(\tau)=\frac{1}{2} \theta_{2}^{6} \theta_{3}^{2}(2 \tau)
$$

Generalizing the identity (3.23), we may now use these theta series to decompose the product of two level-1 theta series into a sum of products of level-2 theta series:

$$
\begin{aligned}
O_{16}^{2}= & \theta_{D_{8}[2] ; O}^{2}+\theta_{D_{8}[2] ; O}^{2}+\theta_{D_{8}[2] ; O}^{2}+\theta_{D_{8}[2] ; O}^{2} \\
& +56 \theta_{D_{8}[2] ; 120}^{2}+135 \theta_{D_{8}[2] ; 1820}^{2}+56 \theta_{D_{8}[2] ; 8008}^{2} \\
V_{16}^{2}= & 2 \theta_{D_{8}[2] ; O} \theta_{D_{8}[2] ; V}+2 \theta_{D_{8}[2] ; S} \theta_{D_{8}[2] ; C} \\
& +56 \theta_{D_{8}[2] ; 120}^{2}+135 \theta_{D_{8}[2] ; 1820}^{2}+56 \theta_{D_{8}[2] ; 8008}^{2} \\
S_{16}^{2}= & 2 \theta_{D_{8}[2] ; O} \theta_{D_{8}[2] ; S}+2 \theta_{D_{8}[2] ; V} \theta_{D_{8}[2] ; C} \\
& +112 \theta_{D_{8}[2] ; 120} \theta_{D_{8}[2] ; 8008}+135 \theta_{D_{8}[2] ; 1820}^{2} \\
C_{16}^{2}= & 2 \theta_{D_{8}[2] ; O} \theta_{D_{8}[2] ; C}+2 \theta_{D_{8}[2] ; V} \theta_{D_{8}[2] ; S} \\
& +112 \theta_{D_{8}[2] ; 120} \theta_{D_{8}[2] ; 8008}+135 \theta_{D_{8}[2] ; 1820}^{2}
\end{aligned}
$$

As in (3.25), we view each term on the right hand side as the product of the partition function for the lattice of physical electric charges $P_{1}+P_{2}$, times the partition function of the lattice of unphysical electric charges $P_{1}-P_{2}$. It is the latter which, together with the partition function of the oscillators, determines the degeneracies of DH states.

In all cases, the level-2 theta series with characteristics are modular forms of weight 4. Taking into account the action on the left-moving bosonic oscillators, we find that the degeneracies in the untwisted sector are enumerated by

$$
\frac{1}{4}\left(\frac{\theta_{D_{8}[2], \lambda}}{\eta^{24}} \pm \delta_{0, \mathcal{P}} \frac{2^{4} \theta_{2}^{4}}{\eta^{12}}\right)
$$

where $\lambda$ is any element in the finite group $D_{8} / 2 D_{8}$, while those in the twisted sectors are counted by

$$
\frac{1}{2}\left(\frac{1}{\eta^{12} \vartheta_{4}^{4}} \pm \frac{1}{\eta^{12} \vartheta_{3}^{4}}\right)
$$


In particular, the asymptotics are governed by the same formulae (3.30). As in any $\mathcal{N}=4$ heterotic models, the absolute degeneracies are equal to ( $2 / 3$ times $)$ the helicity supertraces $\Omega_{4}$.

\section{$\operatorname{Het}(4,12)$ model}

A similar construction as in (D.6) allows to construct the point of enhanced symmetry $S O(8)^{4}$ of the $S O(32)$ heterotic string: one simply needs to orbifold the heterotic string compactified on $S_{1}^{\prime} \times S_{2}^{\prime \prime}$ by $\mathbb{Z}_{2} \times \mathbb{Z}_{2}$, where the two generators $g_{1}$ and $g_{2}$ both act by shifting the $U(1)$ charges of a different set of 8 left-moving bosons ( 4 of which being common to $g_{1}$ and $g_{2}$ ), and by a translation by half a period in either of the two circles. The partition function of the $\Gamma_{2,18}$ Narain lattice at the $S O(8)^{4}$ point is therefore

$$
\begin{aligned}
Z_{D_{4}^{4}}= & \frac{1}{4 \eta^{16}}\left[\frac{1}{2} Z_{2,2}\left[\begin{array}{l}
00 \\
00
\end{array}\right] \sum_{a, b=0,1} \theta^{16}\left[\begin{array}{l}
a \\
b
\end{array}\right]\right. \\
& \left.+\sum_{d d}\left(Z_{2,2}\left[\begin{array}{l}
00 \\
d d
\end{array}\right] \theta_{3}^{8} \theta_{4}^{8}+Z_{2,2}\left[\begin{array}{l}
d d \\
00
\end{array}\right] \theta_{2}^{8} \theta_{3}^{8}+Z_{2,2}\left[\begin{array}{l}
d d \\
d d
\end{array}\right] \theta_{2}^{8} \theta_{4}^{8}\right)\right]
\end{aligned}
$$

where the sum runs over the 2-digit binary numbers $d d=00,0 \frac{1}{2}, \frac{1}{2} 0, \frac{1}{2} \frac{1}{2}$ [49]. Using (D.1), this may be decomposed into characters of $S O(8)^{4}$,

$$
\begin{aligned}
Z_{D_{4}^{4}} & =Z_{++}^{00}\left(O_{8}^{4}+V_{8}^{4}+S_{8}^{4}+C_{8}^{4}\right)+2\left[Z_{+-}^{00}+Z_{-+}^{00}+Z_{--}^{00}\right]\left(O_{8}^{2} V_{8}^{2}+S_{8}^{2} C_{8}^{2}\right) \\
& +\left[Z_{++}^{0 \frac{1}{2}}+Z_{++}^{\frac{1}{2} 0}+Z_{++}^{\frac{1}{2} \frac{1}{2}}+Z_{--}^{\frac{1}{2} \frac{1}{2}}+Z_{+-}^{\frac{1}{2} 0}+Z_{-+}^{0 \frac{1}{2}}\right]\left(O_{8}^{2}+V_{8}^{2}\right)\left(S_{8}^{2}+C_{8}^{2}\right) \\
& +4\left[Z_{--}^{0 \frac{1}{2}}+Z_{--}^{0 \frac{1}{2}}+Z_{-+}^{\frac{1}{2} 0}+Z_{-+}^{\frac{1}{2} \frac{1}{2}}+Z_{+-}^{0 \frac{1}{2}}+Z_{+-}^{\frac{1}{2} \frac{1}{2}}\right] O_{8} V_{8} S_{8} C_{8}
\end{aligned}
$$

where

$$
Z_{\epsilon_{1} \epsilon_{2}}^{h_{1} h_{2}}=\frac{1}{4 \eta^{16}}\left(Z_{2,2}\left[\begin{array}{l}
\frac{h_{1}}{2} \frac{h_{2}}{2} \\
00
\end{array}\right]+\epsilon_{1} Z_{2,2}\left[\begin{array}{l}
\frac{h_{1}}{2} \frac{h_{2}}{2} \\
\frac{1}{2} 0
\end{array}\right]+\epsilon_{2} Z_{2,2}\left[\begin{array}{l}
\frac{h_{1}}{2} \frac{h_{2}}{2} \\
0 \frac{1}{2}
\end{array}\right]+\epsilon_{1} \epsilon_{2} Z_{2,2}\left[\begin{array}{l}
\frac{h_{1}}{2} \frac{h_{2}}{2} \\
\frac{1}{2} \frac{1}{2}
\end{array}\right]\right)
$$

denotes the projected lattice sum in the $\left(h_{1}, h_{2}\right)$ twisted sector of the $\mathbb{Z}_{2} \times \mathbb{Z}_{2}$ orbifold. The resulting theory can be orbifolded by an element $g_{3}:=e$ of order 4 permuting the four $S O(8)$ factors cyclically, together with a translation of order 4 along one of the circles in the torus $T^{4}$. The partition function in the untwisted sector, with an insertion of an odd power of the generator is thus given by

$$
\begin{aligned}
Z_{D_{4}[4]}\left[\begin{array}{l}
0 \\
\frac{g}{4}
\end{array}\right]=\frac{1}{16 \eta^{4}(4 \tau)}\left(Z_{3,3}\left[\begin{array}{l}
000 \\
00 \frac{g}{4}
\end{array}\right]\right. & \left.+Z_{3,3}\left[\begin{array}{l}
000 \\
\frac{1}{2} 0 \frac{g}{4}
\end{array}\right]+Z_{3,3}\left[\begin{array}{l}
000 \\
0 \frac{1}{2} \frac{g}{4}
\end{array}\right]+Z_{3,3}\left[\begin{array}{l}
000 \\
\frac{1}{2} \frac{1}{2} \frac{g}{4}
\end{array}\right]\right) \\
& \times\left[O_{8}(4 \tau)+V_{8}(4 \tau)+S_{8}(4 \tau)+C_{8}(4 \tau)\right]
\end{aligned}
$$


with $g=1,3$, while for an insertion of $e^{2}$,

$$
\begin{aligned}
& Z_{D_{4}[4]}\left[\begin{array}{l}
0 \\
\frac{1}{2}
\end{array}\right]=\frac{1}{8 \eta^{8}(2 \tau)}\left\{\frac{1}{2}\left(Z_{3,3}\left[\begin{array}{l}
000 \\
00 \frac{1}{2}
\end{array}\right]+Z_{3,3}\left[\begin{array}{l}
000 \\
\frac{1}{2} 0 \frac{1}{2}
\end{array}\right]+Z_{3,3}\left[\begin{array}{l}
000 \\
0 \frac{1}{2} \frac{1}{2}
\end{array}\right]+Z_{3,3}\left[\begin{array}{l}
000 \\
\frac{1}{2} \frac{1}{2} \frac{1}{2}
\end{array}\right]\right)\right. \\
& \times\left[O_{8}^{2}(2 \tau)+V_{8}^{2}(2 \tau)+S_{8}^{2}(2 \tau)+C_{8}^{2}(2 \tau)\right] \\
& +\left(3 Z_{3,3}\left[\begin{array}{l}
000 \\
00 \frac{1}{2}
\end{array}\right]-Z_{3,3}\left[\begin{array}{l}
000 \\
\frac{1}{2} 0 \frac{1}{2}
\end{array}\right]-Z_{3,3}\left[\begin{array}{l}
000 \\
0 \frac{1}{2} \frac{1}{2}
\end{array}\right]-Z_{3,3}\left[\begin{array}{l}
000 \\
\frac{1}{2} \frac{1}{2} \frac{1}{2}
\end{array}\right]\right) \\
& \times\left[O_{8}(2 \tau) V_{8}(2 \tau)+S_{8}(2 \tau) C_{8}(2 \tau)\right] \\
& +\left(Z_{3,3}\left[\begin{array}{c}
0 \frac{1}{2} 0 \\
00 \frac{1}{2}
\end{array}\right]+Z_{3,3}\left[\begin{array}{c}
0 \frac{1}{2} 0 \\
0 \frac{1}{2} \frac{1}{2}
\end{array}\right]+Z_{3,3}\left[\begin{array}{c}
\frac{1}{2} 00 \\
00 \frac{1}{2}
\end{array}\right]+Z_{3,3}\left[\begin{array}{c}
\frac{1}{2} 00 \\
\frac{1}{2} 0 \frac{1}{2}
\end{array}\right]+Z_{3,3}\left[\begin{array}{c}
\frac{1}{2} \frac{1}{2} 0 \\
00 \frac{1}{2}
\end{array}\right]+Z_{3,3}\left[\begin{array}{c}
\frac{1}{2} \frac{1}{2} 00 \\
\frac{1}{2} \frac{1}{2} \frac{1}{2}
\end{array}\right]\right) \\
& \left.\times\left[O_{8}(2 \tau)+V_{8}(2 \tau)\right]\left[S_{8}(2 \tau)+C_{8}(2 \tau)\right]\right\}
\end{aligned}
$$

and, in the absence of any insertion, $Z_{D_{4}[4]}\left[\begin{array}{l}0 \\ 0\end{array}\right]=\frac{1}{4} Z_{D_{4}^{4}}$. The twisted sectors can be obtained as usual by modular transformations, leading to

$$
\begin{aligned}
Z_{D_{4}[4]}\left[\begin{array}{c}
\frac{h}{4} \\
\frac{g}{4}
\end{array}\right]=\frac{e^{-2 \pi i g / 3}}{16}\left(Z_{3,3}\left[\begin{array}{c}
00 \frac{h}{4} \\
00 \frac{g}{4}
\end{array}\right]+Z_{3,3}\right. & {\left.\left[\begin{array}{c}
\frac{h}{2} 0 \frac{h}{4} \\
\frac{g}{2} 0 \frac{g}{4}
\end{array}\right]+Z_{3,3}\left[\begin{array}{c}
0 \frac{h}{2} \frac{h}{4} \\
0 \frac{g}{2} \frac{g}{4}
\end{array}\right]+Z_{3,3}\left[\begin{array}{c}
\frac{h}{2} \frac{h}{2} \frac{h}{4} \\
\frac{g}{2} \frac{g}{2}
\end{array}\right]\right) } \\
& \times\left[\frac{O_{8}+V_{8}+S_{8}+C_{8}}{\eta^{4}}\right]\left(\frac{\tau+g}{4}\right)
\end{aligned}
$$

for $h=1,3, g=0,1,2,3$,

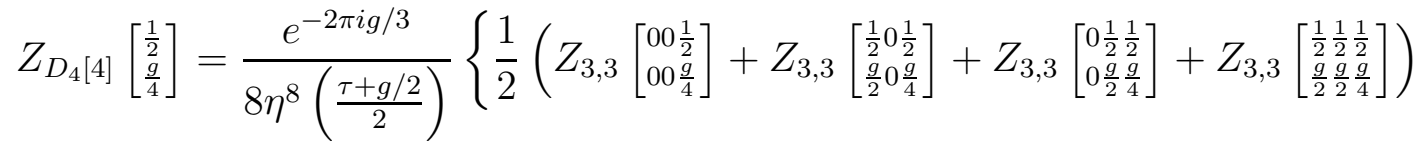

$$
\begin{aligned}
& \times\left[O_{8}^{2}+V_{8}^{2}+S_{8}^{2}+C_{8}^{2}\right]\left(\frac{\tau+g / 2}{2}\right) \\
& +\left(3 Z_{3,3}\left[\begin{array}{l}
00 \frac{1}{2} \\
00 \frac{g}{4}
\end{array}\right]-Z_{3,3}\left[\begin{array}{l}
\frac{1}{2} 0 \frac{1}{2} \\
\frac{g}{2} 0 \frac{g}{4}
\end{array}\right]-Z_{3,3}\left[\begin{array}{l}
0 \frac{1}{2} \frac{1}{2} \\
0 \frac{g}{2} \frac{g}{4}
\end{array}\right]-Z_{3,3}\left[\begin{array}{c}
\frac{1}{2} \frac{1}{2} \frac{1}{2} \\
\frac{g}{2} \frac{g}{2} \frac{g}{4}
\end{array}\right]\right) \times\left[O_{8} V_{8}+S_{8} C_{8}\right]\left(\frac{\tau+g / 2}{2}\right) \\
& +\left(Z_{3,3}\left[\begin{array}{l}
00 \frac{1}{2} \\
0 \frac{g+1}{2} \frac{g}{4}
\end{array}\right]+Z_{3,3}\left[\begin{array}{l}
0 \frac{1}{2} \frac{1}{2} \\
0 \frac{g+1}{2} 0
\end{array}\right]+Z_{3,3}\left[\begin{array}{l}
00 \frac{1}{2} \\
\frac{g+1}{2} 0 \frac{g}{4}
\end{array}\right]+Z_{3,3}\left[\begin{array}{l}
\frac{1}{2} 0 \frac{1}{2} \\
\frac{g+1}{2} 00
\end{array}\right]\right. \\
& \left.\left.+Z_{3,3}\left[\begin{array}{l}
00 \frac{1}{2} \\
\frac{g+1}{2} \frac{g+1}{2} \frac{g}{4}
\end{array}\right]+Z_{3,3}\left[\begin{array}{l}
\frac{1}{2} \frac{1}{2} \frac{1}{2} \\
\frac{g+1}{2} \frac{g+1}{2} 0
\end{array}\right]\right) \times\left[O_{8}+V_{8}\right]\left[S_{8}+C_{8}\right]\left(\frac{\tau+g / 2}{2}\right)\right\}
\end{aligned}
$$

for $g=0,2$ and

$$
\begin{aligned}
& Z_{D_{4}[4]}\left[\begin{array}{c}
\frac{1}{2} \\
\frac{g}{4}
\end{array}\right]=\frac{e^{-2 \pi i g / 3}}{8 \eta^{4}\left(\frac{\tau+(g-1) / 2}{2}\right)}\left\{\frac{1}{2}\left(Z_{3,3}\left[\begin{array}{l}
00 \frac{1}{2} \\
00 \frac{g}{4}
\end{array}\right]+Z_{3,3}\left[\begin{array}{c}
\frac{1}{2} 0 \frac{1}{2} \\
\frac{g}{2} 0 \frac{g}{4}
\end{array}\right]+Z_{3,3}\left[\begin{array}{c}
0 \frac{1}{2} \frac{1}{2} \\
0 \frac{g}{2} \frac{g}{4}
\end{array}\right]+Z_{3,3}\left[\begin{array}{c}
\frac{1}{2} \frac{1}{2} \frac{1}{2} \\
\frac{g}{2} \frac{g}{2} \frac{g}{4}
\end{array}\right]\right)\right. \\
& \times\left[O_{8}+V_{8}+S_{8}+C_{8}\right]\left(\frac{\tau+(g-1) / 2}{2}\right)
\end{aligned}
$$


for $g=1,3$.

In order to extract the degeneracies of states of given electric charge under the invariant (level 4) $S O(8)$, one may work in two steps. Let us first assume that the four charge vectors $P_{1}, \ldots P_{4}$ are in the root lattice of $D_{4}$, as in the first term of (D.22). We decompose

$$
\begin{array}{ll}
P_{1}+P_{3}=2 \Sigma+\mathcal{P}, & P_{2}+P_{4}=2 \Sigma^{\prime}+\mathcal{P}^{\prime} \\
P_{1}-P_{3}=2 \Delta-\mathcal{P}, & P_{2}-P_{4}=2 \Delta^{\prime}-\mathcal{P}^{\prime}
\end{array}
$$

where $\mathcal{P}, \mathcal{P}^{\prime}$ take value in the finite group $D_{4} / 2 D_{4}$. Next we further decompose

$$
\begin{aligned}
& \Sigma+\Sigma^{\prime}=2 \Sigma^{\prime \prime}+\mathcal{P}^{\prime \prime} \\
& \Sigma-\Sigma^{\prime}=2 \Delta^{\prime \prime}-\mathcal{P}^{\prime \prime}
\end{aligned}
$$

where $\mathcal{P}^{\prime \prime} \in D_{4} / 2 E_{4}$. Since the physical charge is

$$
Q=P_{1}+P_{2}+P_{3}+P_{4}=4 \Sigma^{\prime \prime}+2 \mathcal{P}^{\prime \prime}+\mathcal{P}+\mathcal{P}^{\prime}
$$

we obtain the degeneracies of DH states with a given charge $Q$ by summing over $\Delta, \Delta^{\prime}, \Delta^{\prime \prime}, \mathcal{P}, \mathcal{P}^{\prime}, \mathcal{P}^{\prime \prime}$ at fixed values of $\Sigma^{\prime \prime}$ and $\boldsymbol{q}_{0}=\mathcal{P}^{\prime \prime} / 2+\left(\mathcal{P}+\mathcal{P}^{\prime}\right) / 4$ in the discrete group $D_{4} / 4 D_{4}$. For the other terms in (D.22), the same decomposition holds, upon shifting $\mathcal{P}, \mathcal{P}^{\prime}$, or $\mathcal{P}^{\prime \prime}$ by $2 \lambda$ where $\lambda$ is in the weight lattice of $D_{4}$. Decomposing the square of the charge vector as

$$
\begin{aligned}
\sum_{i=1}^{4} P_{i}^{2}= & 2\left(\Delta-\frac{1}{2} \mathcal{P}\right)^{2}+2\left(\Delta^{\prime}-\frac{1}{2} \mathcal{P}^{\prime}\right)^{2} \\
& +4\left(\Delta^{\prime \prime}-\frac{\mathcal{P}^{\prime \prime}}{2}+\frac{\mathcal{P}-\mathcal{P}^{\prime}}{4}\right)^{2}+4\left(\Sigma^{\prime \prime}+\frac{\mathcal{P}^{\prime \prime}}{2}+\frac{\mathcal{P}+\mathcal{P}^{\prime}}{4}\right)^{2}
\end{aligned}
$$

we see that the partition function of the Narain lattice $\Gamma_{2,18}$ at the $S O(8)^{4}$ point may be written as a sum of products of two level 2 theta series

$$
\Theta_{D_{4}[2], \mathcal{P}}(\tau):=\sum_{\Delta \in D_{4}(1)}=e^{2 \pi i \tau\left(\Delta-\frac{1}{2} \mathcal{P}\right)^{2}}
$$

times two level 4 theta series,

$$
\Theta_{D_{4}[4], \mathcal{P}}(\tau):=\sum_{\Delta \in D_{4}(1)}=e^{4 \pi i \tau\left(\Delta-\frac{1}{4} \mathcal{P}\right)^{2}}
$$

corresponding to sums over the lattice vectors $\Delta, \Delta^{\prime}, \Delta^{\prime \prime}, \Sigma^{\prime \prime}$ respectively. Under this decomposition, the last factor can be viewed as the partition function for the physical charges. 
In order to compute the required theta series, note that $D_{4} / 2 D_{4}$ decomposes into five orbits, of respective length $1,1,1,1,12$ : (i) the zero orbit (ii) the orbit of one half the highest weights of the $V, S, C$ irreps and (iii) the orbit of the highest weight of the adjoint representation $A$. The corresponding level 2 theta series are given by

$$
\begin{gathered}
\theta_{D_{4}[2] ; O}(\tau)=O_{8}(2 \tau), \quad \theta_{D_{4}[2] ; V}(\tau)=V_{8}(2 \tau) \\
\theta_{D_{4}[2] ; S}(\tau)=S_{8}(2 \tau), \theta_{D_{4}[2] ; C}(\tau)=C_{8}(2 \tau), \quad \theta_{D_{4}[2] ; A}(\tau)=\frac{1}{2} \theta_{2}^{2} \theta_{3}^{2}(2 \tau)
\end{gathered}
$$

These can then be used to decompose the product of two identical level 1 theta series according to

$$
\begin{aligned}
& O_{8}^{2}=\theta_{D_{4}[2] ; O}^{2}+\theta_{D_{4}[2] ; V}^{2}+\theta_{D_{8}[2] ; S}^{4}+\theta_{D_{8}[2] ; C}^{4}+12 \theta_{D_{8}[2] ; A}^{2} \\
& V_{8}^{2}=2 \theta_{D_{4}[2] ; O} \theta_{D_{4}[2] ; V}+2 \theta_{D_{4}[2] ; S} \theta_{D_{4}[2] ; C}-12 \theta_{D_{8}[2] ; A}^{2}
\end{aligned}
$$

as well as the relations which follow from (D.40) by triality.

The level 4 theta series (D.36) can now be obtained by repeating this procedure twice. They fall into $5 \times 5$ orbits of the Weyl group, corresponding to the two-stage decomposition $D_{4} / 4 D_{4}=\left(D_{4} / 2 D_{4}\right) \times 2 D_{4} / 4 D_{4}$. The theta series corresponding to $\mathcal{P}=4 \lambda$ where $\lambda$ is the highest weight of the $O, S, C, V, A$ representations are simply obtained from (D.37) by doubling the argument $\tau \rightarrow 2 \tau$.

Using the duplication identities in Appendix B, one may rewrite the partition functions of the oscillators in the untwisted sector as

$$
\frac{1}{\eta^{8}(\tau) \eta^{4}(4 \tau)}=\frac{2^{4}}{\left(\theta_{3}^{2}-\theta_{4}^{2}\right) \theta_{2} \eta^{9}}, \quad \frac{1}{\eta^{8}(\tau) \eta^{8}(2 \tau)}=\frac{2^{4}}{\theta_{2}^{4} \eta^{12}}
$$

We thus find that the degeneracies in the untwisted sector are enumerated by

$$
\begin{aligned}
\frac{1}{16} \sum_{\substack{\mathcal{P}, \mathcal{P}^{\prime}, \mathcal{P}^{\prime \prime} \in D_{4} / 2 D_{4}, \lambda \\
\mathcal{P}+\mathcal{P}^{\prime}+2 \mathcal{P}^{\prime \prime}+\lambda=\mathcal{P}_{0}}} & \left(\frac{\theta_{D_{4}[2], \mathcal{P}+2 \lambda} \theta_{D_{8}[2], \mathcal{P}^{\prime}+2 \lambda} \theta_{D_{4}[4],-\mathcal{P}+\mathcal{P}^{\prime}+2 \mathcal{P}^{\prime \prime}}}{\eta^{24}}\right. \\
& \left. \pm \delta_{0, \mathcal{P}} \delta_{0, \mathcal{P}^{\prime}} \delta_{0, \lambda} 2^{4} \frac{\theta_{D_{4}[2], \mathcal{P}^{\prime \prime}}(2 \tau)}{\theta_{2}^{4} \eta^{12}} \pm \delta_{0, \mathcal{P}} \delta_{0, \mathcal{P}^{\prime}} \delta_{0, \mathcal{P}^{\prime \prime}} \delta_{\lambda, 0} \frac{2^{4}}{\theta_{2}\left(\theta_{3}^{2}-\theta_{4}^{2}\right) \eta^{9}}\right)
\end{aligned}
$$

The three terms behaves as

$$
\hat{I}_{7}\left(4 \pi \sqrt{Q^{2} / 2}\right), \hat{I}_{7}\left(4 \pi \sqrt{\frac{1}{2} Q^{2} / 2}\right), \hat{I}_{7}\left(4 \pi \sqrt{\frac{3}{8} Q^{2} / 2}\right)
$$

respectively, so that the degeneracies are dominated by the untwisted, unprojected contribution. 
In the sector twisted by the order 2 element $e^{2}$, the momenta automatically have $\Delta=\mathcal{P}=0$ and $\Delta^{\prime}=\mathcal{P}^{\prime}=0$ but one still needs to sum over the unphysical charges $\Delta^{\prime \prime}$ using the level 2 identities (D.39) with $\tau \rightarrow \tau / 2$. Using the duplication identities

$$
\frac{1}{\eta^{8}(\tau) \eta^{4}(\tau / 2)}=\frac{2^{2}}{\theta_{4}^{2} \eta^{10}}, \quad \frac{1}{\eta^{8}(\tau) \eta^{8}(\tau / 2)}=\frac{2^{4}}{\theta_{4}^{4} \eta^{12}}
$$

we find that the degeneracies are given by

$$
\frac{1}{2}\left(\frac{1}{\eta^{12} \vartheta_{4}^{4}} \pm \frac{1}{\eta^{12} \vartheta_{3}^{4}}\right) \theta_{D_{8}[2], \mathcal{P}^{\prime \prime}}(\tau / 2)+\delta_{0, \mathcal{P}^{\prime \prime}}\left(\frac{1}{\eta^{10} \vartheta_{4}^{2}} \pm \frac{1}{\eta^{10} \vartheta_{3}^{2}}\right)
$$

Finally, in the sectors twisted by the order 4 element $e$ or $e^{3}$, one may rewrite the partition functions for the twisted oscillators as

$$
\begin{gathered}
\frac{1}{\eta^{8}(\tau) \eta^{4}(4 \tau)}=\frac{2^{4}}{\left(\theta_{3}^{2}-\theta_{4}^{2}\right) \theta_{2} \eta^{9}} \\
\frac{1}{\eta^{8}(\tau) \eta^{4}\left(\frac{\tau}{4}\right)}=\frac{1}{\left(\theta_{3}^{2}-\theta_{2}^{2}\right) \theta_{4} \eta^{9}} \quad, \frac{1}{\eta^{8}(\tau) \eta^{4}\left(\frac{\tau+1}{4}\right)}=\frac{e^{i \pi / 12}}{\left(\theta_{4}^{2}-i \theta_{2}^{2}\right) \theta_{3} \eta^{9}} \\
\frac{1}{\eta^{8}(\tau) \eta^{4}\left(\frac{\tau+2}{4}\right)}=\frac{e^{i \pi / 6}}{\left(\theta_{3}^{2}+\theta_{2}^{2}\right) \theta_{4} \eta^{9}} \quad, \frac{1}{\eta^{8}(\tau) \eta^{4}\left(\frac{\tau+3}{4}\right)}=\frac{e^{i \pi / 4}}{\left(\theta_{4}^{2}+i \theta_{2}^{2}\right) \theta_{4} \eta^{9}}
\end{gathered}
$$

We thus find that the degeneracies are enumerated by

$$
\frac{1}{4}\left(\frac{1}{\left(\theta_{3}^{2}-\theta_{2}^{2}\right) \theta_{4} \eta^{9}}+\epsilon_{1} \frac{1}{\left(\theta_{4}^{2}-i \theta_{2}^{2}\right) \theta_{3} \eta^{9}}+\epsilon_{2} \frac{1}{\left(\theta_{3}^{2}+\theta_{2}^{2}\right) \theta_{4} \eta^{9}}+\epsilon_{3} \frac{1}{\left(\theta_{4}^{2}+i \theta_{2}^{2}\right) \theta_{3} \eta^{9}}\right)
$$

where, depending on the moding of the momenta along the three circles, $\left(\epsilon_{1}, \epsilon_{2}, \epsilon_{3}\right)$ is any vector in $(1,1,1),(-i,-1, i),(-1,1,-1),(i,-1,-i)$, (the corresponding ground state dimensions are $\Delta=3 / 8,1 / 8,-1 / 8, \Delta=-3 / 8$, respectively.)

In all sectors, applying the Rademacher formula we find that the degeneracies grow uniformly as

$$
\Omega_{a b s}(Q)=\frac{2}{3} \Omega_{4}(Q) \sim \hat{I}_{7}\left(4 \pi \sqrt{Q^{2} / 2}\right)+\cdots
$$

The exponentially suppressed corrections however depend sensitively on the details of the charges.

\section{A Het $(2,8)$ model}

Let us now consider an $\mathcal{N}=2$ variant of the $(4,12)$ model. We start from the $S O(32)$ heterotic string on $T^{2}$ at the point of enhanced symmetry $S O(8)^{4}$, further compactify on a square $T^{4}$,

$$
\Gamma_{6,22}=D_{4}(-1) \oplus D_{4}(-1) \oplus D_{4}(-1) \oplus D_{4}(-1) \oplus I I^{2,2} \oplus I I^{4,4}
$$


and perform a $\mathbb{Z}^{4}$ orbifold acting on the momenta as

$$
g\left|P_{1}, P_{2}, P_{3}, P_{4}, P_{5}, P_{6}\right\rangle=e^{2 \pi i \delta \cdot P_{5}}\left|P_{2}, P_{3}, P_{4}, P_{1}, P_{5}, R(g) P_{6}\right\rangle
$$

where $R(g)$ acts by a $\mathbb{Z}_{4}$ rotation in a two-plane inside $T^{4}$, breaking the supersymmetry to $\mathcal{N}=2$. The degeneracies can be obtained easily from the $(4,12)$ model by dropping the untwisted, unprojected sector and multiplying by $\eta^{4}$ times the partition function of four $\mathbb{Z}_{4}$-twisted left-moving bosons (the contribution of the right-moving bosons is absorbed into the helicity supertrace). The orbifold blocks for four $\mathbb{Z}_{4}$-twisted chiral bosons can be obtained by the following simple trick: Consider the orbifold of $4 \times 4=16$ chiral bosons by cyclic permutations of the four blocks of four. The partition function with one insertion of the $\mathbb{Z}_{4}$ generator is $1 / \eta^{4}(4 \tau)$. On the other hand, diagonalizing the oscillators, it should be the product of four untwisted, four $\mathbb{Z}_{2}$-twisted boson and eight $\mathbb{Z}_{4}$-twisted chiral bosons:

$$
\frac{1}{\eta^{4}(4 \tau)}=\frac{1}{\eta^{4}(\tau)} \times \frac{2^{2} \eta^{2}(\tau)}{\theta_{2}^{2}(\tau)} \times\left(Z_{4}\left[\begin{array}{c}
0 \\
\frac{1}{4}
\end{array}\right]\right)^{2}
$$

hence

$$
Z_{4}\left[\begin{array}{c}
0 \\
\frac{1}{4}
\end{array}\right]=Z_{4}\left[\begin{array}{c}
0 \\
\frac{3}{4}
\end{array}\right]=\frac{\eta^{2}(2 \tau)}{\eta^{2}(4 \tau)}=4 \sqrt{\frac{\eta \theta_{2}}{\theta_{3}^{2}-\theta_{4}^{2}}}=\frac{2 \eta}{\theta\left[\begin{array}{c}
\frac{1}{2} \\
\frac{1}{4}
\end{array}\right](0 \mid \tau)}
$$

The other orbifold blocks can be obtained by modular transformations,

$$
\begin{gathered}
Z_{4}\left[\begin{array}{c}
\frac{1}{4} \\
\frac{g}{4}
\end{array}\right]=Z_{4}\left[\begin{array}{c}
\frac{3}{4} \\
\frac{g}{4}
\end{array}\right]=2 \frac{\eta^{2}((\tau+g) / 2)}{\eta^{2}((\tau+g) / 4)}, \\
Z_{4}\left[\begin{array}{l}
0 \\
0
\end{array}\right]=\frac{Z_{4,4}}{\eta^{4}}, Z_{4}\left[\begin{array}{c}
0 \\
\frac{1}{2}
\end{array}\right]=\frac{\eta^{4}(\tau)}{\eta^{4}(2 \tau)}, \quad Z_{4}\left[\begin{array}{c}
\frac{1}{2} \\
0
\end{array}\right]=4 \frac{\eta^{4}(\tau)}{\eta^{4}(\tau / 2)}, \\
Z_{4}\left[\begin{array}{c}
\frac{1}{2} \\
\frac{1}{4}
\end{array}\right]=2 \frac{\eta^{2}(\tau)}{\eta^{2}(\tau / 2)}, \quad Z_{4}\left[\begin{array}{c}
\frac{1}{2} \\
\frac{1}{2}
\end{array}\right]=4 \frac{\eta^{4}(\tau+1)}{\eta^{4}((\tau+1) / 2)}, \quad Z_{4}\left[\begin{array}{c}
\frac{1}{2} \\
\frac{3}{4}
\end{array}\right]=2 \frac{\eta^{2}(\tau+1)}{\eta^{2}((\tau+1) / 2)}
\end{gathered}
$$

Using the same notation as in the $(4,12)$ model, we thus find that the second helicity supertraces $\Omega_{2}$ in the untwisted sector are generated by

$$
\frac{1}{16} \delta_{0, \mathcal{P}} \delta_{0, \mathcal{P}^{\prime}} 2^{6} \frac{\theta_{D_{4}[2], \mathcal{P}^{\prime \prime}}(2 \tau)}{\theta_{2}^{6} \eta^{6}} \pm \delta_{0, \mathcal{P}} \delta_{0, \mathcal{P}^{\prime}} \delta_{\mathcal{P}^{\prime \prime}, 0} \frac{2^{6}}{\sqrt{\theta_{2}\left(\theta_{3}^{2}-\theta_{4}^{2}\right)^{3} \eta^{9}}}
$$

Importantly, the untwisted unprojected term does not contribute, due to its extended $\mathcal{N}=4$ supersymmetry. The second term grows as

$$
\hat{I}_{5}\left(4 \pi \sqrt{\frac{3}{16} Q^{2} / 2}\right)
$$


and is suppressed with respect to the first.

Finally, in the sector twisted by the order 2 element, we find that the second helicity supertraces are generated by

$$
\frac{1}{2}\left(\frac{1}{\eta^{6} \vartheta_{4}^{6}} \pm \frac{1}{\eta^{6} \vartheta_{3}^{6}}\right) \theta_{D_{8}[2], \mathcal{P}^{\prime \prime}}(\tau / 2)+\delta_{0, \mathcal{P}^{\prime \prime}}\left(\frac{1}{\eta^{4} \vartheta_{4}^{4}} \pm \frac{1}{\eta^{4} \vartheta_{3}^{4}}\right)
$$

The degeneracies from the second term grow as

$$
\hat{I}_{5}\left(4 \pi \sqrt{\frac{2}{3} Q^{2} / 2}\right)
$$

Finally, in the sectors twisted by the order 4 element $e$ or $e^{3}$, we find that the second helicity supertraces are enumerated by

$$
\frac{1}{\sqrt{\left(\theta_{3}^{2}-\theta_{2}^{2}\right)^{3} \theta_{4} \eta^{9}}}+\epsilon_{1} \frac{1}{\sqrt{\left(\theta_{4}^{2}-i \theta_{2}^{2}\right)^{3} \theta_{3} \eta^{9}}}+\epsilon_{2} \frac{1}{\sqrt{\left(\theta_{3}^{2}+\theta_{2}^{2}\right)^{3} \theta_{4} \eta^{9}}}+\epsilon_{3} \frac{1}{\sqrt{\left(\theta_{4}^{2}+i \theta_{2}^{2}\right)^{3} \theta_{3} \eta^{9}}}
$$

where $\left(\epsilon_{1}, \epsilon_{2}, \epsilon_{3}\right)$ is any vector in $(1,1,1),(-i,-1, i),(-1,1,-1),(i,-1,-i)$. The corresponding ground state energies are $\Delta=3 / 8,1 / 8,-1 / 8, \Delta=-3 / 8$ respectively. In these four cases, the second helicity supertraces grow as

$$
\hat{I}_{5}\left(4 \pi \sqrt{Q^{2} / 2}\right)
$$

\section{E. Some Properties of the Mac-Mahon Function}

In this section, we derive some properties of the Mac-Mahon function

$$
f(\lambda):=\sum_{n=1}^{\infty} n \log \left(1-q^{n}\right)
$$

with $q=e^{i n \lambda}$. This is an entire function of $\lambda$ in the upper half plane. Taylor-expanding the logarithm and carrying out the sum over $n$, it may be rewritten as

$$
f(\lambda)=\sum_{d=1}^{\infty} \frac{1}{d} \frac{1}{\left(2 \sin \frac{d \lambda}{2}\right)^{2}}
$$

We would like to derive the asymptotic expansion for $\lambda \rightarrow 0$.

Let us recall the standard argument. From the standard expansion

$$
\frac{x}{e^{x}-1}=1-\frac{x}{2}+\sum_{n \geq 1} \frac{B_{2 n}}{(2 n) !} x^{2 n}
$$


in terms of the Bernoulli numbers $B_{n}$, we get

$$
\frac{1}{(2 \sin (x / 2))^{2}}=\frac{1}{x^{2}}+\sum_{n \geq 1} \frac{2 n-1}{(2 n) !} B_{2 n}(-1)^{n-1} x^{2 n-2}
$$

Note that $B_{2 n}=(-1)^{n-1}\left|B_{2 n}\right|$.

If we substitute (E.4) into (E.2) and exchange the sum on $n$ and $d$ we find the series

$$
\lambda^{-2} \zeta(3)+\sum_{n=1}^{\infty} \frac{(2 n-1)\left|B_{2 n}\right|}{(2 n) !} \lambda^{2 n-2}\left(\sum_{d \geq 1} d^{2 n-3}\right)
$$

Note that the sums on $d$ are infinite. While one may try and define them for $n \neq 1$ by zeta function regularization, the $n=1$ term is still infinite. If we simply discard the $n=1$ term and use this regularization we get

$$
\lambda^{-2} \zeta(3)-\sum_{n=0}^{\infty} \lambda^{2 n+2} \frac{\left|B_{2 n+4}\right|}{(2 n+4) !} \frac{(2 n+3)}{(2 n+2)} B_{2 n+2}
$$

Using the relations between Bernoulli numbers and Rieman zeta functions,

$$
\zeta(3-2 g)=-\frac{B_{2 g-2}}{2 g-2}, \quad \zeta(2 g)=(-1)^{g+1} \frac{B_{2 g} 2^{2 g-1} \pi^{2 g}}{(2 g) !}
$$

valid for $g \geq 2, g \geq 1$, respectively, one recovers the standard result in the topological string literature.

However the manipulation used above is not valid. One way to see it is that an entire function such as $f(\lambda)$ cannot possibly have an infinite term $\lambda^{0} \zeta(1)$ in its asymptotics. Nevertheless, the amazing agreement between the coefficients of the terms $\lambda^{\geq 2}$ with the integrals on moduli space [71] and with the predictions of heterotic/typeII duality [73] suggest the higher terms are indeed correct. This will prove to be the case.

One valid way to derive the asymptotics is to proceed as follows. We use the series

$$
\frac{1}{\sin ^{2}(\pi x)}=\frac{1}{\pi^{2}} \sum_{n \in \mathbb{Z}} \frac{1}{(x+n)^{2}}
$$

Substituting into (E.2), the double sum on $n, d$ is absolutely convergent. We can therefore exchange the sum on $n, d$. Defining $z:=\lambda /(2 \pi)$ we have

$$
f(\lambda)=\frac{\zeta(3)}{\lambda^{2}}+\frac{1}{4 \pi^{2}} \sum_{n \neq 0} \sum_{d=1}^{\infty} \frac{1}{d(d z+n)^{2}}
$$

Define

$$
g(z)=\frac{1}{4 \pi^{2}} \sum_{n \neq 0} \sum_{d=1}^{\infty} \frac{1}{d(d z+n)^{2}}
$$


In order to study the $z \rightarrow 0$ asymptotics, we should apply the Poisson summation formula to the sum on $d$.

Care is however needed due to the incomplete summation on $d$. While the Poisson summation formula as usually stated applies to continuous functions, we wish to apply it to the function

$$
f(x):= \begin{cases}\frac{1}{x(x z+n)^{2}} & x \geq 1 \\ 0 & x<1\end{cases}
$$

This falls off nicely at infinity, but has a discontinuity at $x=1$.

Suppose, generally, that $f(x)$ has a discontinuity at $x=1$. The standard procedure to prove the Poisson formula is to construct the periodic function $F(x)=$ $\sum_{n \in \mathbb{Z}} f(x+n)$, expand it in Fourier series, $F(x)=\sum_{m \in \mathbb{Z}} \hat{F}_{m} e^{2 \pi i m x}$, and evaluate at $x=0$. For piecewise continuous functions, the Fourier series only converges to the average $\frac{1}{2}(F(0+)+F(0-))$ at points of discontinuity. If $f(x)=0$ for $x<1$ then we get

$$
\frac{1}{2} f(1)+\sum_{d=2}^{\infty} f(d)=\sum_{\ell \in \mathbb{Z}} \int_{1}^{\infty} e^{2 \pi i \ell x} f(x) d x
$$

Taking this into account we have the Poisson summation formula

$$
\sum_{d=1}^{\infty} \frac{1}{d(d z+n)^{2}}=\frac{1}{2(z+n)^{2}}+\sum_{\ell \in \mathbb{Z}} \int_{1}^{\infty} e^{2 \pi i \ell x} \frac{1}{x(x z+n)^{2}} d x
$$

Now we write $g(z)=g_{0}(z)+g_{1}(z)$ where

$$
\begin{aligned}
& g_{0}(z):=\frac{1}{4 \pi^{2}} \sum_{n \neq 0}\left(\frac{1}{2(z+n)^{2}}+\int_{1}^{\infty} \frac{1}{x(x z+n)^{2}} d x\right) \\
& g_{1}(z):=\frac{1}{4 \pi^{2}} \sum_{n \neq 0} \sum_{\ell \neq 0} \int_{1}^{\infty} e^{2 \pi i \ell x} \frac{1}{x(x z+n)^{2}} d x
\end{aligned}
$$

To compute the integrals we write

$$
\frac{1}{x(x z+n)^{2}}=-\frac{z}{n(x z+n)^{2}}+\frac{1}{x n^{2}}-\frac{z}{(x z+n) n^{2}}=\frac{d}{d x}\left[\frac{1}{n^{2}} \log \frac{x}{x z+n}+\frac{1}{n(x z+n)}\right]
$$

Let us analyze first $g_{0}(z)$. The integral on $x$ is elementary and we get:

$$
g_{0}(z)=\frac{1}{4 \pi^{2}} \sum_{n \neq 0}\left(\frac{1}{2(z+n)^{2}}+\frac{1}{n^{2}}\left[\log (1 / z)-\log \left(\frac{1}{z+n}\right)\right]-\frac{1}{n(z+n)}\right)
$$


Expanding the various terms and recalling that $z=\lambda /(2 \pi)$ we find

$$
g_{0}(z)=\frac{1}{12} \log \frac{2 \pi}{\lambda}+\frac{i \pi}{24}+\frac{1}{4 \pi^{2}} \sum_{n \geq 1} \frac{\log n^{2}}{n^{2}}+\sum_{n=0}^{\infty} \lambda^{2 n+2} \frac{\left|B_{2 n+4}\right|}{(2 n+4) !}\left(n+\frac{1}{2}-\frac{1}{2 n+2}\right)
$$

or, equivalently,

$$
g_{0}(z)=\frac{1}{12} \log \frac{2 \pi i}{\lambda}-\frac{1}{2 \pi^{2}} \zeta^{\prime}(2)+\sum_{n=0}^{\infty} \lambda^{2 n+2} \frac{\left|B_{2 n+4}\right|}{(2 n+4) !}\left(n+\frac{1}{2}-\frac{1}{2 n+2}\right)
$$

Now we turn to (E.16). We can write $g_{1}$ as a sum of three terms:

$$
\begin{aligned}
g_{1}(z) & =h_{1}+h_{2}+h_{3} \\
h_{1}(z) & :=\frac{1}{4 \pi^{2}} \sum_{n \neq 0} \sum_{\ell \neq 0} \frac{1}{n^{2}} \int_{1}^{\infty} e^{2 \pi i \ell x} \frac{1}{x} d x \\
h_{2}(z) & :=-\frac{1}{4 \pi^{2}} \sum_{n \neq 0} \sum_{\ell \neq 0} \frac{z}{n^{2}} \int_{1}^{\infty} e^{2 \pi i \ell x} \frac{1}{x z+n} d x \\
h_{3}(z) & :=-\frac{1}{4 \pi^{2}} \sum_{n \neq 0} \sum_{\ell \neq 0} \frac{z}{n} \int_{1}^{\infty} e^{2 \pi i \ell x} \frac{1}{(x z+n)^{2}} d x
\end{aligned}
$$

The first term, $h_{1}$ is just a constant in $z$, but is only convergent when we group together the $\ell$ and $-\ell$ terms in the sum. The integral can be computed in terms of the cosine integral function $\mathrm{Ci}(x)$ defined in [102] 5.2.27:

$$
h_{1}=\frac{1}{4 \pi^{2}} \frac{\pi^{2}}{3} \sum_{\ell=1}^{\infty} \int_{1}^{\infty} 2 \cos (2 \pi \ell x) \frac{d x}{x}=-\frac{1}{6} \sum_{\ell=1}^{\infty} \mathrm{Ci}(2 \pi \ell)
$$

Since $\operatorname{Ci}(2 \pi x) \sim 1 /(2 \pi x)^{2}$ for large integer $x$, the sum over $\ell$ converges. Indeed, $h_{1}=\frac{1}{12} \gamma_{E}$ where $\gamma_{E}$ is the Euler-Mascharoni constant.

For the second term we use the identity 5.1.28 in [102]:

$$
\int_{1}^{\infty} e^{2 \pi i \ell x} \frac{1}{x z+n} d x=\frac{1}{z} e^{-2 \pi i \ell(n / z)} E_{1}(-2 \pi i \ell(1+n / z))
$$

Note that $z$ has a nonzero imaginary part so the argument of the exponential integral, and the denominator in the integral is never zero even if $n$ is negative $\left(E_{1}\right.$ is a variant of the exponential integral). Then we use the asymptotic expansion AS 5.1.51 to get

$$
\int_{1}^{\infty} e^{2 \pi i \ell x} \frac{1}{x z+n} d x \sim \frac{1}{z} \sum_{s=0}^{\infty} \frac{(-1)^{s} s !}{(-2 \pi i \ell(1+n / z))^{s+1}}
$$


For $z$ pure imaginary, $z \rightarrow 0$, say, this is in the valid range for the expansion. Now we sum over $\ell$ and get:

$$
\sum_{\ell \neq 0} \int_{1}^{\infty} e^{2 \pi i \ell x} \frac{1}{x z+n} d x \sim \sum_{k=0}^{\infty} \frac{(-1)^{k}\left|B_{2 k+2}\right|}{2 k+2} \frac{z^{2 k+1}}{(z+n)^{2 k+2}}
$$

Taking a derivative with respect to $n$ gives

$$
\sum_{\ell \neq 0} \int_{1}^{\infty} e^{2 \pi i \ell x} \frac{1}{(x z+n)^{2}} d x \sim \sum_{k=0}^{\infty}(-1)^{k}\left|B_{2 k+2}\right| \frac{z^{2 k+1}}{(z+n)^{2 k+3}}
$$

Next we expand the denominators in a power series in $z / n$ and include the sum over $n$. In this way we get:

$$
\begin{aligned}
& h_{2} \sim-\sum_{n=0}^{\infty} \lambda^{2 n+2} \frac{\left|B_{2 n+4}\right|}{(2 n+4) !} \frac{1}{2 n+2} \sum_{k=0}^{n}\left(\begin{array}{l}
2 n+2 \\
2 k+2
\end{array}\right) B_{2 k+2} \\
& h_{3} \sim-\sum_{n=0}^{\infty} \lambda^{2 n+2} \frac{\left|B_{2 n+4}\right|}{(2 n+4) !} \sum_{k=0}^{n}\left(\begin{array}{l}
2 n+2 \\
2 k+2
\end{array}\right) B_{2 k+2}
\end{aligned}
$$

Now, the Bernoulli polynomial $B_{n}(x)=\sum_{k=0}^{n}\left(\begin{array}{l}n \\ k\end{array}\right) B_{k} x^{n-k}$ at $x=1$ is $B_{n}(1)=(-1)^{n} B_{n}$ so we may simplify

$$
h_{2}+h_{3} \sim-\sum_{n=0}^{\infty} \lambda^{2 n+2} \frac{\left|B_{2 n+4}\right|}{(2 n+4) !}\left(\frac{2 n+3}{2 n+2} B_{2 n+2}+\frac{2 n+3}{2 n+2} n\right)
$$

Putting it all together, the asymptotics for $f(\lambda)$ for $\lambda \rightarrow 0$ in the upper half-plane are

$$
f(\lambda) \sim \lambda^{-2} \zeta(3)-\sum_{n=0}^{\infty} \lambda^{2 n+2} \frac{\left|B_{2 n+4}\right|}{(2 n+4) !} \frac{(2 n+3)}{(2 n+2)} B_{2 n+2}+\frac{1}{12} \log \frac{2 \pi i}{\lambda}-\frac{1}{2 \pi^{2}} \zeta^{\prime}(2)+\frac{1}{12} \gamma_{E}
$$

This differs from the standard expression by the last three terms. While the constant is not so important, the logarithmic term is indeed important.

We close this section by an observation which hints at possibly interesting modular properties of the Mac-Mahon function. By analogy with the Dedekind $\eta$ function, let us compute

$$
E_{3}(\tau):=-q \frac{d}{d q} f(\lambda)=\sum_{n=1}^{\infty} \frac{n^{2} q^{n}}{1-q^{n}}
$$


where $q=e^{2 \pi i \tau}=e^{i n \lambda}$ (the reason for this notation will become clear shortly). Expanding the denominators, we obtain

$$
E_{3}(\tau)=\sum_{N=1}^{\infty}\left(\sum_{n \mid N} n^{2}\right) q^{N}=\sum_{n=1}^{\infty} \sum_{m=1}^{\infty} n^{2} q^{m n}=\sum_{m=1}^{\infty} \frac{q^{m}\left(1+q^{m}\right)}{\left(1-q^{m}\right)^{3}}
$$

Now we use the identity

$$
\sum_{n \in \mathbb{Z}} \frac{1}{(n+z)^{3}}=4 i \pi^{3} \frac{p(1+p)}{(1-p)^{3}}
$$

where $p=e^{2 \pi i z}$. This allows to rewrite

$$
E_{3}(\tau)=\frac{1}{4 i \pi^{3}} \sum_{m=1}^{\infty} \sum_{n=-\infty}^{\infty} \frac{1}{(n+m \tau)^{3}}
$$

While this expression is similar to the usual modular invariant Eisenstein series $E_{2 n}$, it is important to note that, due to the restriction $m>0, E_{3}$ is not modular invariant. Instead, its orbit under $S l(2, \mathbb{Z})$ is an infinite family of functions

$$
E_{3}^{(p, q)}(\tau)=\frac{1}{4 i \pi^{3}} \sum_{(m, n) \in \mathbb{Z}, p m+n q>0} \frac{1}{(n+m \tau)^{3}}
$$

In particular, $E_{3}(\tau)=E(\tau)=E_{3}^{(1,0)}(\tau)$ is mapped under $\tau \rightarrow-1 / \tau$ to $E_{3}^{(0,1)}(\tau)$ which does not admit a $q$-expansion. Indeed, $f(\lambda)$ at $\lambda \rightarrow 0$ is not exponentially suppressed but rather consists of an infinite power series, as discussed above.

\section{References}

[1] A. Dabholkar, F. Denef, G. W. Moore and B. Pioline, Exact and asymptotic degeneracies of small black holes, hep-th/0502157.

[2] J. D. Bekenstein, Black holes and entropy, Phys. Rev. D7 (1973) 2333-2346.

[3] J. D. Bekenstein, Generalized second law of thermodynamics in black hole physics, Phys. Rev. D9 (1974) 3292-3300.

[4] S. W. Hawking, Particle creation by black holes, Commun. Math. Phys. 43 (1975) 199-220.

[5] A. Strominger and C. Vafa, Microscopic Origin of the Bekenstein-Hawking Entropy, Phys. Lett. B379 (1996) 99-104 [hep-th/9601029]. 
[6] J. M. Maldacena and A. Strominger, Statistical entropy of four-dimensional extremal black holes, Phys. Rev. Lett. 77 (1996) 428-429 [hep-th/9603060].

[7] C. V. Johnson, R. R. Khuri and R. C. Myers, Entropy of 4 D Extremal Black Holes, Phys. Lett. B378 (1996) 78-86 [hep-th/9603061].

[8] R. M. Wald, Black hole entropy in the Noether charge, Phys. Rev. D48 (1993) 3427-3431 [gr-qc/9307038].

[9] V. Iyer and R. M. Wald, Some properties of Noether charge and a proposal for dynamical black hole entropy, Phys. Rev. D50 (1994) 846-864 [gr-qc/9403028].

[10] G. Lopes Cardoso, B. de Wit and T. Mohaupt, Corrections to macroscopic supersymmetric black-hole entropy, Phys. Lett. B451 (1999) 309-316 [hep-th/9812082].

[11] G. Lopes Cardoso, B. de Wit and T. Mohaupt, Deviations from the area law for supersymmetric black holes, Fortsch. Phys. 48 (2000) 49-64 [hep-th/9904005].

[12] G. Lopes Cardoso, B. de Wit and T. Mohaupt, Area law corrections from state counting and supergravity, Class. Quant. Grav. 17 (2000) 1007-1015 [hep-th/9910179].

[13] G. Lopes Cardoso, B. de Wit, J. Kappeli and T. Mohaupt, Stationary BPS solutions in $N=2$ supergravity with $R^{2}$ interactions, JHEP 12 (2000) 019 [hep-th/0009234].

[14] H. Ooguri, A. Strominger and C. Vafa, Black hole attractors and the topological string, hep-th/0405146.

[15] A. Sen, How does a fundamental string stretch its horizon?, hep-th/0411255.

[16] A. Sen, Black holes, elementary strings and holomorphic anomaly, hep-th/0502126.

[17] A. Sen, Black Holes and the Spectrum of Half-BPS States in N=4 Supersymmetric String Theory, hep-th/0504005.

[18] G. L. Cardoso, B. de Wit, J. Kappeli and T. Mohaupt, Asymptotic degeneracy of dyonic $N=4$ string states and black hole entropy, hep-th/0412287.

[19] M. Bershadsky, S. Cecotti, H. Ooguri and C. Vafa, Holomorphic anomalies in topological field theories, Nucl. Phys. B405 (1993) 279-304 [hep-th/9302103].

[20] I. Antoniadis, E. Gava, K. S. Narain and T. R. Taylor, Topological amplitudes in string theory, Nucl. Phys. B413 (1994) 162-184 [hep-th/9307158].

[21] A. Sen, Black hole entropy function and the attractor mechanism in higher derivative gravity, hep-th/0506177. 
[22] S. Ferrara, R. Kallosh and A. Strominger, N=2 extremal black holes, Phys. Rev. D52 (1995) 5412-5416 [hep-th/9508072].

[23] S. Ferrara and R. Kallosh, Supersymmetry and attractors, Phys. Rev. D54 (1996) 1514-1524 [hep-th/9602136].

[24] S. Ferrara and R. Kallosh, Universality of supersymmetric attractors, Phys. Rev. D54 (1996) 1525-1534 [hep-th/9603090].

[25] C. Vafa, Two dimensional Yang-Mills, black holes and topological strings, hep-th/0406058.

[26] M. Aganagic, H. Ooguri, N. Saulina and C. Vafa, Black holes, q-deformed $2 d$ Yang-Mills, and non-perturbative topological strings, hep-th/0411280.

[27] A. Dabholkar and J. A. Harvey, Nonrenormalization of the superstring tension, Phys. Rev. Lett. 63 (1989) 478.

[28] A. Dabholkar, G. W. Gibbons, J. A. Harvey and F. Ruiz Ruiz, Superstrings and solitons, Nucl. Phys. B340 (1990) 33-55.

[29] L. Susskind, Some speculations about black hole entropy in string theory, hep-th/9309145.

[30] L. Susskind and J. Uglum, Black hole entropy in canonical quantum gravity and superstring theory, Phys. Rev. D50 (1994) 2700-2711 [hep-th/9401070].

[31] J. G. Russo and L. Susskind, Asymptotic level density in heterotic string theory and rotating black holes, Nucl. Phys. B437 (1995) 611-626 [hep-th/9405117].

[32] A. Sen, Extremal black holes and elementary string states, Mod. Phys. Lett. A10 (1995) 2081-2094 [hep-th/9504147].

[33] G. T. Horowitz and J. Polchinski, A correspondence principle for black holes and strings, Phys. Rev. D55 (1997) 6189-6197 [hep-th/9612146].

[34] A. Sen, Black hole solutions in heterotic string theory on a torus, Nucl. Phys. B440 (1995) 421-440 [hep-th/9411187].

[35] A. Dabholkar, J. P. Gauntlett, J. A. Harvey and D. Waldram, Strings as solitons and black holes as strings, Nucl. Phys. B474 (1996) 85-121 [hep-th/9511053].

[36] A. Dabholkar, R. Kallosh and A. Maloney, A stringy cloak for a classical singularity, JHEP 12 (2004) 059 [hep-th/0410076].

[37] A. Dabholkar, Exact counting of black hole microstates, hep-th/0409148. 
[38] K. Behrndt et. al., Classical and quantum $n=2$ supersymmetric black holes, Nucl. Phys. B488 (1997) 236-260 [hep-th/9610105].

[39] K. Behrndt et. al., Higher-order black-hole solutions in $n=2$ supergravity and calabi-yau string backgrounds, Phys. Lett. B429 (1998) 289-296 [hep-th/9801081].

[40] V. Hubeny, A. Maloney and M. Rangamani, String-corrected black holes, hep-th/0411272.

[41] J. A. Harvey and G. W. Moore, Fivebrane instantons and $R^{2}$ couplings in $N=4$ string theory, Phys. Rev. D57 (1998) 2323-2328 [hep-th/9610237].

[42] D. Bak, S. Kim and S.-J. Rey, Exactly soluble BPS black holes in higher curvature $N$ $=2$ supergravity, hep-th/0501014.

[43] R. Dijkgraaf, J. M. Maldacena, G. W. Moore and E. Verlinde, A black hole Farey tail, hep-th/0005003.

[44] J. M. Maldacena, A. Strominger and E. Witten, Black hole entropy in M-theory, JHEP 12 (1997) 002 [hep-th/9711053].

[45] E. Verlinde, Attractors and the holomorphic anomaly, hep-th/0412139.

[46] R. Dijkgraaf, R. Gopakumar, H. Ooguri and C. Vafa, Baby universes in string theory, hep-th/0504221.

[47] P. Kraus and F. Larsen, Microscopic black hole entropy in theories with higher derivatives, hep-th/0506176.

[48] C. Vafa, Black holes and Calabi-Yau threefolds, Adv. Theor. Math. Phys. 2 (1998) 207-218 [hep-th/9711067].

[49] E. Kiritsis, N. A. Obers and B. Pioline, Heterotic/type II triality and instantons on K3, JHEP 01 (2000) 029 [hep-th/0001083].

[50] M. Cvetic and A. A. Tseytlin, General class of BPS saturated dyonic black holes as exact superstring solutions, Phys. Lett. B366 (1996) 95-103 [hep-th/9510097].

[51] M. Bershadsky, C. Vafa and V. Sadov, D-Branes and Topological Field Theories, Nucl. Phys. B463 (1996) 420-434 [hep-th/9511222].

[52] S.-T. Yau and E. Zaslow, BPS States, String Duality, and Nodal Curves on K3, Nucl. Phys. B471 (1996) 503-512 [hep-th/9512121].

[53] G. B. Arfken and H. J. Weber, Mathematical methods for physicists, . Acacemic Press (2000) $1112 \mathrm{P}$. 
[54] I. Antoniadis, B. Pioline and T. R. Taylor, Calculable e $e^{-1 / l a m b d a}$ effects, Nucl. Phys. B512 (1998) 61-78 [hep-th/9707222].

[55] E. Witten, Quantum background independence in string theory, hep-th/9306122.

[56] E. Kiritsis, Introduction to non-perturbative string theory, hep-th/9708130.

[57] J. A. Harvey and G. W. Moore, Algebras, BPS States, and Strings, Nucl. Phys. B463 (1996) 315-368 [hep-th/9510182].

[58] S. Ferrara and C. Kounnas, Extended supersymmetry in four-dimensional type II strings, Nucl. Phys. B328 (1989) 406.

[59] S. Chaudhuri, G. Hockney and J. D. Lykken, Maximally supersymmetric string theories in D i 10, Phys. Rev. Lett. 75 (1995) 2264-2267 [hep-th/9505054].

[60] S. Chaudhuri and J. Polchinski, Moduli space of CHL strings, Phys. Rev. D52 (1995) 7168-7173 [hep-th/9506048].

[61] A. Gregori et. al., $r^{2}$ corrections and non-perturbative dualities of $N=4$ string ground states, Nucl. Phys. B510 (1998) 423-476 [hep-th/9708062].

[62] A. Dabholkar and J. A. Harvey, String islands, JHEP 02 (1999) 006 [hep-th/9809122].

[63] J. H. Schwarz and A. Sen, Type IIA dual of the six-dimensional CHL compactification, Phys. Lett. B357 (1995) 323-328 [hep-th/9507027].

[64] S. Chaudhuri and D. A. Lowe, Type IIA heterotic duals with maximal supersymmetry, Nucl. Phys. B459 (1996) 113-124 [hep-th/9508144].

[65] J. Distler and G. Moore, unpublished.

[66] A. Sen and C. Vafa, Dual pairs of type II string compactification, Nucl. Phys. B455 (1995) 165-187 [hep-th/9508064].

[67] B. Pioline, BPS black hole degeneracies and minimal automorphic representations, hep-th/0506228.

[68] T. Mohaupt, Black hole entropy, special geometry and strings, Fortsch. Phys. 49 (2001) 3-161 [hep-th/0007195].

[69] M. Bershadsky, S. Cecotti, H. Ooguri and C. Vafa, Kodaira-Spencer theory of gravity and exact results for quantum string amplitudes, Commun. Math. Phys. 165 (1994) 311-428 [hep-th/9309140]. 
[70] P. Candelas, X. C. De La Ossa, P. S. Green and L. Parkes, A pair of Calabi-Yau manifolds as an exactly soluble superconformal theory, Nucl. Phys. B359 (1991) 21-74.

[71] C. Faber and R. Pandharipande, Hodge integrals and Gromov-Witten theory, math. AG/9810173.

[72] I. Antoniadis, E. Gava, K. S. Narain and T. R. Taylor, N=2 type II heterotic duality and higher derivative F terms, Nucl. Phys. B455 (1995) 109-130 [hep-th/9507115].

[73] M. Marino and G. W. Moore, Counting higher genus curves in a Calabi-Yau manifold, Nucl. Phys. B543 (1999) 592-614 [hep-th/9808131].

[74] A. Klemm, M. Kreuzer, E. Riegler and E. Scheidegger, Topological string amplitudes, complete intersection Calabi-Yau spaces and threshold corrections, hep-th/0410018.

[75] R. Gopakumar and C. Vafa, M-theory and topological strings. I, hep-th/9809187.

[76] R. Gopakumar and C. Vafa, M-theory and topological strings. II, hep-th/9812127.

[77] G. Lopes Cardoso, B. de Wit and T. Mohaupt, Macroscopic entropy formulae and non-holomorphic corrections for supersymmetric black holes, Nucl. Phys. B567 (2000) 87-110 [hep-th/9906094].

[78] R. Minasian, G. W. Moore and D. Tsimpis, Calabi-Yau black holes and (0,4) sigma models, Commun. Math. Phys. 209 (2000) 325-352 [hep-th/9904217].

[79] N. Seiberg and E. Witten, The D1/D5 system and singular CFT, JHEP 04 (1999) 017 [hep-th/9903224].

[80] J. A. Harvey and G. W. Moore, Exact gravitational threshold correction in the FHSV model, Phys. Rev. D57 (1998) 2329-2336 [hep-th/9611176].

[81] M. Henningson and G. W. Moore, Threshold corrections in K3 $\times T^{2}$ heterotic string compactifications, Nucl. Phys. B482 (1996) 187-212 [hep-th/9608145].

[82] M. Shmakova, Calabi-Yau black holes, Phys. Rev. D56 (1997) 540-544 [hep-th/9612076].

[83] S. Kachru and C. Vafa, Exact results for $N=2$ compactifications of heterotic strings, Nucl. Phys. B450 (1995) 69-89 [hep-th/9505105].

[84] G. Aldazabal, A. Font, L. E. Ibanez and F. Quevedo, Chains of N=2, $D=4$ heterotic/type II duals, Nucl. Phys. B461 (1996) 85-100 [hep-th/9510093].

[85] S. Ferrara, J. A. Harvey, A. Strominger and C. Vafa, Second quantized mirror symmetry, Phys. Lett. B361 (1995) 59-65 [hep-th/9505162]. 
[86] S. D. Miller and G. W. Moore, Landau-Siegel zeroes and black hole entropy, hep-th/9903267.

[87] S. S. Gelbart and S. D. Miller, Riemann's zeta function and beyond, Bull. Amer. Math. Soc. 4159.

[88] R. Minasian and G. W. Moore, K-theory and Ramond-Ramond charge, JHEP 11 (1997) 002 [hep-th/9710230].

[89] D. S. Freed and E. Witten, Anomalies in string theory with D-branes, hep-th/9907189.

[90] I. Brunner, M. R. Douglas, A. E. Lawrence and C. Romelsberger, D-branes on the quintic, JHEP 08 (2000) 015 [hep-th/9906200].

[91] M. R. Douglas, B. Fiol and C. Romelsberger, Stability and BPS branes, hep-th/0002037.

[92] M. R. Douglas, D-branes, categories and $N=1$ supersymmetry, J. Math. Phys. 42 (2001) 2818-2843 [hep-th/0011017].

[93] E. Witten, BPS bound states of D0-D6 and DO-D8 systems in a B-field, JHEP 04 (2002) 012 [hep-th/0012054].

[94] F. Denef, Supergravity flows and D-brane stability, JHEP 08 (2000) 050 [hep-th/0005049].

[95] F. Denef, Quantum quivers and Hall/hole halos, JHEP 10 (2002) 023 [hep-th/0206072].

[96] B. Bates and F. Denef, Exact solutions for supersymmetric stationary black hole composites, hep-th/0304094.

[97] F. Bogomolov, Holomorphic tensors and vector bundles on projective varieties, Math. USSR Izv. 13 (1979) 499.

[98] Friedman, Algebraic Surfaces and Holomorphic Vector Bundles. New York, Springer-Verlag, 1998.

[99] S. D. Mathur, The fuzzball proposal for black holes: An elementary review, hep-th/0502050.

[100] I. Bakas and E. Kiritsis, Beyond the large $N$ limit: Nonlinear $W_{\infty}$ as symmetry of the $S L(2, R) / U(1)$ coset model, Int. J. Mod. Phys. A7S1A (1992) 55-81 [hep-th/9109029]. 
[101] J. Distler and P. Nelson, New discrete states of strings near a black hole, Nucl. Phys. B374 (1992) 123-155.

[102] M. Abramowitz and S. I.A., Handbook of Mathematical Functions. New York, Dover Publishing, 1972. 